NOTE TO USERS

This reproduction is the best copy available.

UMI 



\title{
ADVANCES IN INTELLIGENT CONTINUOUS STRUCTURAL HEALTH MONITORING APPLICATIONS OF BRIDGES
}

\author{
A thesis submitted to \\ the Faculty of Graduate Studies and Research \\ in Partial Fulfillment of the requirements for the degree \\ Masters of Applied Science \\ by \\ Ryan O'Connell \\ Department of Civil and Environmental Engineering \\ Carleton University \\ Ottawa-Carleton Institute of Civil and Environmental Engineering \\ September 2009 \\ (C2009 Ryan 0’Connell
}


Library and Archives Canada

Published Heritage Branch

395 Wellington Street Ottawa ON K1A ON4 Canada
Bibliothèque et

Archives Canada

Direction du

Patrimoine de l'édition

395 , rue Wellington

Ottawa ON K1A ON4

Canada
Your file Votre référence

ISBN: 978-0-494-60223-2

Our file Notre référence

ISBN: 978-0-494-60223-2
NOTICE:

The author has granted a nonexclusive license allowing Library and Archives Canada to reproduce, publish, archive, preserve, conserve, communicate to the public by telecommunication or on the Internet, loan, distribute and sell theses worldwide, for commercial or noncommercial purposes, in microform, paper, electronic and/or any other formats.

The author retains copyright ownership and moral rights in this thesis. Neither the thesis nor substantial extracts from it may be printed or otherwise reproduced without the author's permission.

\begin{abstract}
AVIS:
L'auteur a accordé une licence non exclusive permettant à la Bibliothèque et Archives Canada de reproduire, publier, archiver, sauvegarder, conserver, transmettre au public par télécommunication ou par l'Internet, prêter, distribuer et vendre des thèses partout dans le monde, à des fins commerciales ou autres, sur support microforme, papier, électronique etlou autres formats.
\end{abstract}

L'auteur conserve la propriété du droit d'auteur et des droits moraux qui protège cette thèse. $\mathrm{Ni}$ la thèse ni des extraits substantiels de celle-ci ne doivent être imprimés ou autrement reproduits sans son autorisation.
In compliance with the Canadian Privacy Act some supporting forms may have been removed from this thesis.

While these forms may be included in the document page count, their removal does not represent any loss of content from the thesis.
Conformément à la loi canadienne sur la protection de la vie privée, quelques formulaires secondaires ont été enlevés de cette thèse.

Bien que ces formulaires aient inclus dans la pagination, il n'y aura aucun contenu manquant. 


\section{Abstract}

Bridges are an extremely important part of today's infrastructure. In addition to being exposed to severe environmental conditions, a continuing increase in high volume of vehicle traffic and heavy truck loads have led to a higher rate of deterioration in the performance of many old bridges in Canada. The current practice of bridge infrastructure management is mainly based on manual visual inspection, which can lead to subjective and at times unreliable results. Recently the topic of vibration based structural health monitoring has attracted considerable interest as a new technology which offers the possibility of obtaining more accurate and objective information with respect to the deterioration and damage condition of instrumented structures. A sophisticated continuous, real-time remote monitoring system has been developed and installed on the Confederation Bridge in Prince Edward Island in eastern Canada to determine its field vibration properties and behaviour, and the variabilities associated with them, which are essential for the development of reliable structural condition assessment and damage detection techniques for bridges in practical field applications. The vibration component of the monitoring system captures the ambient vibration responses of the bridge due to wind, traffic, ice, and earthquake loads. A robust software platform, Signal Processing Platform for Analysis of Structural Health (SPPLASH), has been developed in a previous phase of the project, encompassing the data processing, 
management, and analysis tools for the determination of the field vibration properties of the structure based on the stochastic subspace identification technique. A new, more efficient system identification module has been developed in the present study based on the newly developed polyreference least square complex frequency algorithm to enhance the real-time automatic data processing and analysis functionality of the monitoring software platform. The clean stabilization diagrams generated by the method allows for development of an automated pole selection procedure which reduces the subjectivity of the results and allows for reliable continuous monitoring and accurate assessment of the condition of a structure. 


\section{Acknowledgements}

I wish to express my gratitude to my supervisor Professor David T. Lau for his time, patience, and enthusiasm during my research. It was a pleasure to work with him and learn from his experiences.

I wish to thank Don McGinn and the staff of Strait Crossing Bridge Limited for their resources and assistance during trips to the Confederation Bridge, as well as John Egan of Public Works and Government Services Canada for his assistance with both maintenance of the data acquisition system and guidance at the bridge.

I would like to thank my family for their encouragement and interest in my studies. Finally, special thanks go to Meagan for her support, patience, and encouragement throughout my studies. 


\section{Table of Contents}

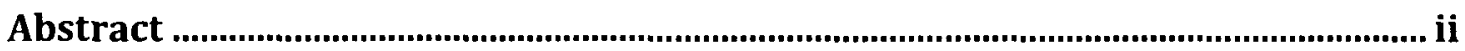

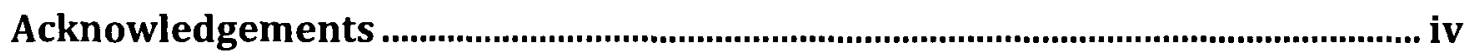

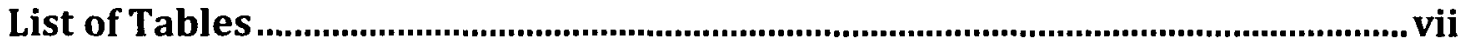

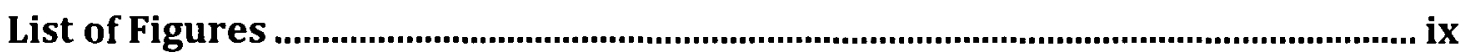

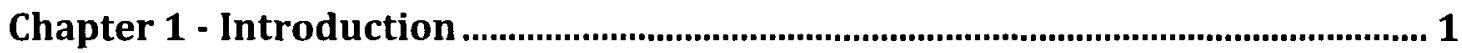

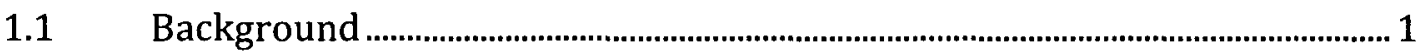

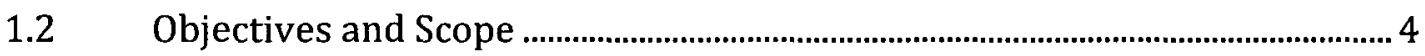

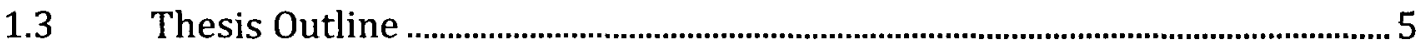

Chapter 2 - Bridge Monitoring Software Development............................................. 7

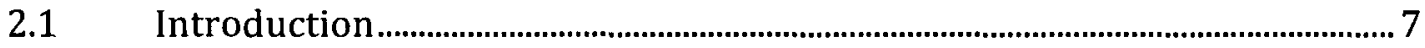

2.2 Current Practice for Data Storage and Use....................................................... 10

2.3 Currently Available Monitoring Software......................................................... 13

Chapter 3 - Confederation Bridge Monitoring Project ............................................ 17

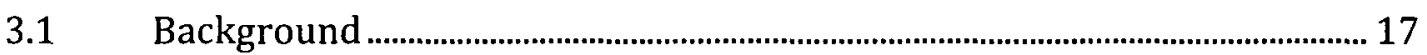

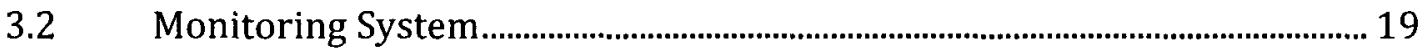

3.3 Signal Processing Platform for Analysis of Structural Health ....................... 22

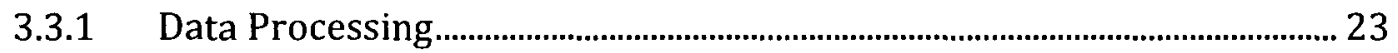

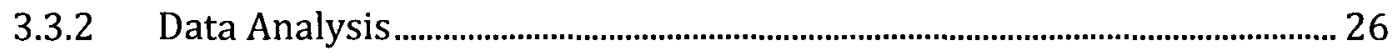

3.3.3 Data Animation and Visualization ................................................................. 27 
4.1 Introduction.............................................................................................................. 43

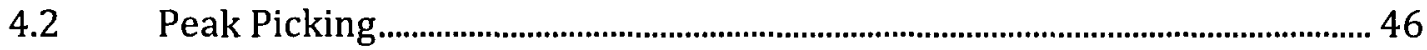

4.3 Stochastic Subspace Identification ............................................................................ 48

4.4 Polyreference Least Squares Complex Frequency Estimator ......................... 54

4.4.1 Validation..............................................................................................................

4.5 Automatic Pole Selection ........................................................................................... 65

4.5.1 Validation..................................................................................................................... 69

Chapter 5 - Variability of Monitoring Data ...................................................................97

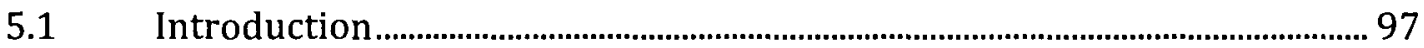

$5.2 \quad$ Baseline Variability ....................................................................................................101

5.3 Baseline Variability Results ......................................................................................102

5.4 Environmental and Loading Variability ..............................................................103

5.5 Environmental and Loading Variability Results ................................................104

Chapter 6 - Conclusions and Recommendations for Future Work..................... 138

6.1 Conclusion …….............................................................................................................138

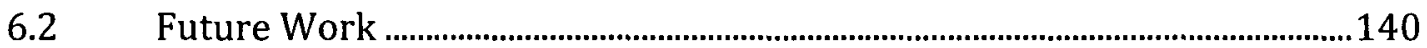

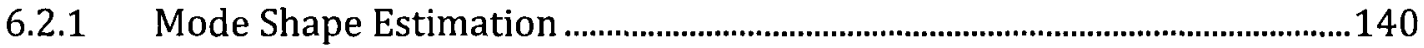

6.2.2 Damage Detection Module ………………………………….......................................141

6.2.3 Engineering Report Module....................................................................................142

References …........................................................................................................................ 14 


\section{List of Tables}

Table 3-1 : Accelerometer locations and channels for Confederation Bridge monitoring project

Table 4-1 : p-LSCF validation results using 3 reference sensors ......................................... 71

Table 4-2 : SSI validation results using 3 references sensors ............................................. 72

Table 4-3 : SSI validation results using 6 reference sensors................................................. 73

Table 4-4 : SSI validation results using 9 references sensors ............................................... 74

Table 4-5 : SSI validation results using 12 reference sensors.............................................. 75

Table 4-6 : SSI validation results using 15 reference sensors................................................. 76

Table 4-7 : Closely spaced poles validation results................................................................. 77

Table 4-8 : Effect of exponential window correction ............................................................. 78

Table 4-9 : p-LSCF estimator results with added white Gaussian noise 79

Table 4-10 : Selected poles based on automatic pole selection procedure ..................... 80

Table 4-11: Selected poles from sine wave data including white Gaussian noise based on automatic pole selection procedure

Table 4-12 : Comparison of user types for selection of poles ............................................. 82

Table 5-1 : Baseline variability results.....................................................................................106

Table 5-2 : Environmental and loading scenario variability results ..............................107 
Table 5-3 : Linear regression results of modal frequency vs. average concrete

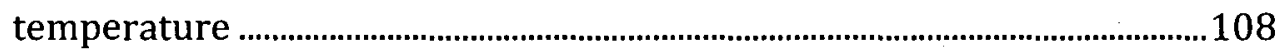




\section{List of Figures}

Figure 3-1 : Location of the Confederation Bridge ................................................................ 29

Figure 3-2 : Typical frame layout of Confederation Bridge .................................................... 30

Figure 3-3 : Locations of thermocouples in Confederation Bridge monitoring system

Figure 3-4 : Typical thermocouple layout of girder in Confederation Bridge monitoring system 32

Figure 3-5: Thermocouple layout of pier in Confederation Bridge monitoring system 33

Figure 3-6: Locations of strain gauges in Confederation Bridge monitoring system

Figure 3-7 : (a) Horizontal displacement sensor locations; (b) Vertical displacement sensor locations. 35

Figure 3-8: Locations of accelerometers in Confederation Bridge monitoring system 36

Figure 3-9: Data flow through Confederation Bridge monitoring system. 37

Figure 3-10 : Removal of baseline offset from measured response data ........................ 38

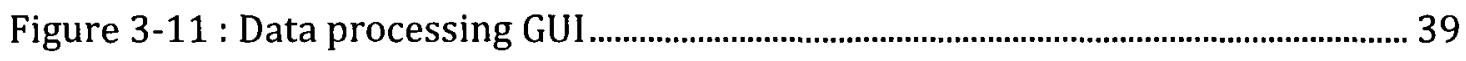

Figure 3-12: Power spectral density of a single sensor 40 
Figure 3-13 : Data analysis GUI displaying stabilization diagram

Figure 3-14 : Bridge response visualization GUI displaying 3D representation of the structure

Figure 4-1: Hamming Window. 83

Figure 4-2 : Time history data before and after application of the Hamming window

Figure $4-3: 1 \%$ Exponential Window. 85

Figure 4-4 : Data correlations before and after application of the exponential window 86

Figure 4-5 : Stabilization diagram based on p-LSCF estimator with $\alpha_{1}=I_{r}$ constraint

Figure 4-6 : Stabilization diagram based on p-LSCF estimator with $\alpha_{n+1}=I_{r}$ constraint 88

Figure 4-7 : Stabilization diagram showing determination of closely spaced poles using p-LSCF estimator 89

Figure 4-8 : Stabilization diagram showing determination of closely spaced poles using SSI 90

Figure 4-9 : Example of validation data with and without white Gaussian noise addition

Figure 4-10 : Stabilization diagram generated by p-LSCF estimator 92

Figure 4-11: Automated pole selection procedure 93 
Figure 4-12(A) : Comparison of selected poles by three user groups 1) Novice, 2) Experienced, 3) Automated 94

Figure 5-1: Accelerometer locations for Confederation Bridge monitoring system

Figure 5-2: Average concrete temperature and average wind speed of baseline variability datasets 110

Figure 5-3(A) : Time histories of baseline variability datasets 111

Figure 5-4(A) : ANPSD of baseline variability datasets. 115

Figure 5-5 : Normalized modal frequencies of baseline variability datasets 118

Figure 5-6 : Normalized damping ratios of baseline variability datasets .119

Figure 5-7(A) : Time histories of environmental and loading scenario variability datasets.

Figure 5-8(A) : ANPSD of environmental and loading scenario variability datasets

Figure 5-9(A) : Normalized frequencies of environmental and load scenario variability data 134

Figure 5-10(A) : Normalized damping ratios of environmental and load scenario variability data .136 


\section{Chapter 1 - Introduction}

\subsection{Background}

Bridges are a very important part of today's infrastructure. Due to their exposure to environmental effects, as well as a continuing increase in high volume of heavy truck loads, a higher rate of deterioration in their performance may occur. The current practice of bridge condition assessment relies mainly on visual inspections, the most basic monitoring technique available. These inspections are typically carried out once every two years (Federal Highway Administration, 2004) and are intended to identify any changes in the conditions of the bridge from previous inspections, while also ensuring the bridge still meets all serviceability requirements set forth by the design codes and standards and operational 
jurisdictions. These inspections are commonly performed by an inspection team, which may or may not have performed previous inspections on the bridge in question. Bridge inspection can be classified into four categories (Fu, 2005):

1) Routine inspections

2) Damage inspections

3) In-depth inspections

4) Special inspections

Routine inspections represent the most frequent type of visual inspections. They are used to assess the general condition of the bridge. A damage inspection is performed when an area of the bridge is known to have suffered some damage and knowledge of the severity of the damage and its effects on the remainder of the structure is needed to be investigated. An in-depth inspection may be required should a damage inspection be incapable of determining the full effects of the known or identified damage. A specialized inspection is carried out specifically related to a specific problem of the structure.

Although visual inspection is the most common technique, it has several shortcomings in its practice. It has been shown that visual inspection results can be influenced by the experience and subjective performance of the inspectors and thus has an impact on the consistency of the information. This variability in the inspection results can be especially significant in the evaluation of the condition of primary elements of bridges where the ratings can have a range of five out of ten $(\mathrm{Fu}, 2005)$. In the case of in-depth inspections, experiences have shown that despite 
the increased level of details in the inspection process all deterioration processes or damage that a structure may have accumulated cannot be identified. While most indepth inspections can identify and locate significant deficiencies, they sometimes miss the presence of minor cracks or signs of deterioration at an early stage (Graybeal et al. 2001). In one study, it is shown that up to $32 \%$ of condition ratings of visually inspected bridges may be inaccurate ( $\mathrm{Li}$ et al. 2008), which calls into question the reliability of the practice of visual inspection of structures. These findings demonstrate the need for improvement in the current practice of condition assessment of bridges, particularly in early detection of deterioration or damage to allow for the implementation of more cost effective mitigation measures.

In order to achieve more objective and reliable condition assessment results, the alternative technology of remote monitoring and vibration based assessment of civil engineering structures has been developed in recent years. Instrumentation of structures provides the opportunity to develop a robust methodology for the continuous, automatic condition assessment of civil engineering structures. On the other hand, sensor instrumentation of a structure generates a large volume of data which require major time consuming effort to process and analyze in order to extract the relevant engineering information. To facilitate this process, a robust software platform incorporating state-of-the-art data processing techniques is needed for the extraction of the desired information from the large volume of monitoring data in a timely and efficient manner. The techniques employed by the software platform should be as user independent as possible, eliminating as much of 
the subjectivity associated with user interaction as possible. To this end a robust software platform, Signal Processing Platform for Analysis of Structural Health, has been developed in the previous phase of the current study by the research group at Carleton University (Desjardins, 2004). Using this software, Londono (2006) has determined the variability of the measured vibration response data, crucial to determining the viability of vibration based structural health monitoring techniques for reliable condition assessment of bridge structures in practical field applications.

\subsection{Objectives and Scope}

The main objective of this thesis is the development of a robust automated pole selection procedure for use in a state-of-the-art software platform intended for efficient monitoring of instrumented bridges. This procedure reduces the subjectivity of the results of the employed system identification technique by eliminating the requirement of user intervention during processing of the monitoring data in the selection of physical poles from a stabilization diagram.

A survey of currently available monitoring software is presented. The need and benefits of open and scalable software to facilitate the adoption of vibration based structural health monitoring technology as an effective alternative approach for bridge infrastructure management by bridge owners and operators are discussed. 
Chapter 1 - Introduction

Finally, the variability of measured vibration response data from an instrumented bridge is examined to establish a general variability baseline for deterioration or damage detection.

\subsection{Thesis Outline}

This thesis is divided into 6 chapters. The following provides a brief description of each chapter.

Chapter 1 presents motivation and objectives of the research. Chapter 2 presents an overview of bridge management and monitoring software for bridges. Chapter 3 describes the Confederation Bridge monitoring project and the existing software platform that has been developed for the project. Chapter 4 discusses advances in vibration based structural health monitoring, with focus on system identification techniques for reliable determination of vibration properties. As well, a new automatic pole selection procedure is presented and implemented in the monitoring software Signal Processing Platform for Analysis of Structural Health for extraction of the modal vibration properties of the instrumented structure from the measured vibration response data. Chapter 6 presents the results and findings of studies on the variability characteristics of monitoring data due to the influences of the site operational environment and environmental conditions. Since the basic 
premise of most damage detection techniques and algorithms is the detection of small changes in the field measured vibration properties of the monitored structure, any inherent variability in the field monitoring data due to other factors not related to change in the condition of the structure would have a significant impact on the sensitivity, accuracy, and reliability of vibration based structural health monitoring as a viable, efficient alternative to manual visual inspection for bridge management. Finally, Chapter 7 presents the concluding remarks of the thesis, along with recommendations for further work. 


\section{Chapter 2 - Bridge Monitoring}

\section{Software Development}

\subsection{Introduction}

Due to recent advances in technology and developments in the practice of instrumentation of bridges, an important method for the examination of the condition of a structure has garnered significant attention as a viable alternative approach to the common practice of periodic manual visual inspection. Remote monitoring of a bridge structure by sensors and intelligent processing of the monitoring data allow real-time updates on the assessment of the condition of the 
instrumented bridge through extraction and analysis of relevant engineering information from the measured response data. As a result of the recent developments in instrumentation and data collection from continuous monitoring of instrumented bridges, there is the new challenge of efficient and timely processing of large amount of data which require considerable effort to manipulate and organize in order to extract the desired information for engineering use. Therefore, a robust data processing and analysis software platform is needed to overcome these limitations which minimize the need of direct intervention by the analyst. The adoption of electronic surveillance of measurements by sensors in bridge maintenance will lead to better informed decision making in bridge operation, and thus ensure safe operation of bridges and better prioritization in the allocation of limited resources for more cost effective bridge maintenance and asset management.

During the design phase of the instrumentation scheme of a bridge monitoring project, the selection of the type and layout of sensors is dependent upon the objectives of the monitoring project. Two main categories exist for measurement techniques:

1) Localized physical phenomena

2) Global structural behaviour

Localized sensors can be installed at specific points of interest to monitor the behaviour of key elements, such as expansion joints or bearings, or they can be distributed near an existing crack or high stress critical area to monitor its growth. 
However, as is evident from the example of crack growth, there are limitations associated with a strictly localized monitoring system. Most importantly, a priori knowledge of the deterioration process and location is necessary in order to establish the proper density, distribution, and location of the sensors.

In contrast, a monitoring system with sensors designed to capture global structural behaviour may be more suitable for detection of the early signs of deterioration or damage. A monitoring system with the proper sensor distribution and density can provide an accurate assessment of the structural condition of the bridge. A detection of a change in the behaviour pattern of an instrumented bridge can be the result of the physical manifestation of deterioration of the structure. Following the detection of a variation in the behaviour pattern of a structure, further damage detection techniques and algorithms, manual field visual inspection or a combination of both actions can then be called upon to diagnose the problem and to further locate the problematic area in the bridge in more refined details. However, global monitoring has some sensitivity issues due to higher levels of noise and uncertainties than that of localized monitoring. Following the determination of the global behaviour of the structure, methods such as finite-element updating can be employed to quantify the extent of the deterioration and determine its location. The problematic region can then be localized so that more detailed inspection methods may be deployed to monitor any further developments. Lessons learned from previous bridge monitoring projects have shown that effective 
instrumentation schemes often include deployment of sensors for both local and global monitoring in a single monitoring system.

\subsection{Current Practice for Data Storage and Use}

Currently, the predominant form of monitoring, based on long established practice, is manual visual inspection, which results in periodic, structure specific data. To facilitate the management of these data and their use for maintenance, repair, and planning purposes, computerized database management software have been developed and are deployed by all major public bridge network jurisdictions. Originally these platforms were designed simply for data archival and retrieval. However, they have evolved to include deterioration models for elements and tools for decision planning. While these tools have increased the usefulness of the recorded data, they remain dependent upon the reliability and accuracy of visual inspection data, which has been drawn into question (Li et al. 2008). To reduce the subjectivity of manual visual inspection based structural condition assessment, remote field monitoring technologies of bridge structures by installed sensors have recently been developed and their advantages have been demonstrated in a number of monitoring projects around the world, such as the Confederation Bridge, Tsing Ma Bridge, and the Shenzhen Bay Highway Bridge. However, because of the usually very large volume of data collected by a bridge monitoring system, efficient 
software is needed to organize, maintain, analyze, and archive the data obtained from instrumented structures. While this type of software platform needed for handling the monitoring data of instrumented bridges is different from the existing bridge inspection database management software, some functionalities of the existing software can still be incorporated in the software system needed for bridge monitoring.

The following is a representative sample of existing bridge management software adopted by bridge owner jurisdictions in North America. Similar systems are employed by jurisdictions throughout the world.

The Ministry of Ontario has developed the Ontario Bridge Management Software (OBMS) to facilitate project based decisions required for the management of its bridge inventory. One of the primary objectives of the software is to integrate the management activities required for the inventory into a single system (Thompson et al. 2003). Upon the input of manual visual inspection results, OBMS provides the user with element, project, and network based cost analysis results, and can develop maintenance and management alternatives for their upkeep. The Nova Scotia Department of Transportation and Public Works has recently implemented a customized version of OBMS to facilitate the management of their bridge inventory following an evaluation which showed that OBMS is best suited for their needs due to the inclusion of features of the Canadian Bridge Design Code in the management platform (Speiran et al. 2004). 
Through the combined efforts of six Western Canadian municipalities, a Bridge Management System software platform was developed to provide cost effective management of their bridge inventory (Kriviak, 1999). The software provides a percentage rating for each bridge in the inventory, along with a prediction of the least costly management strategy for the inventory.

One of the most commonly used bridge management software platforms in the United States is Pontis. Through the use of Pontis, the user can monitor the entire management cycle of a structure from inspection to rehabilitation (Robert et al. 2003). The extensive adoption of the Pontis system by bridge owners and operators in the United States can be attributed to its flexibility and available customization.

Another popular American software platform is BRIDGIT, which has the functionality to assist decision makers with network management decisions and project actions (Hawk, 1999). Through the incorporation of inventory, inspection, and bridge modeling data, an analysis tool is included in this system to develop work plans for inventory of structure(s) based upon user specified policies and practices. 
Chapter 2 - Bridge Monitoring Software Development

\subsection{Currently Available Monitoring Software}

In light of the need for processing of the large volume of data collected by bridge monitoring systems, more advanced software is required for the management of instrumented structures. To facilitate the efficient management and use of the measured data, a robust software platform which incorporates data recording, storage, processing, analysis, and projection is necessary. There exist four main types of software for the management of instrumented structures for the purpose of structural health monitoring and condition assessment:

1) Hardware Manufacturer Supplied

2) Commercially Developed

3) Project Specific

4) Adaptable

Monitoring hardware is often supplied with software by the manufacturer. The software is commonly limited in its capabilities, with a lack of flexibility resulting from the software limitations to interface with specific hardware and/or basic data formats. Often this type of software lacks the capabilities and functionalities required for an extensive monitoring project.

Commercially developed monitoring software platforms are commonly used in mechanical and aeronautical industry. Recently, some of these platforms have been adapted for civil engineering applications, while new platforms have also been 
developed specifically for the purpose of civil engineering applications. BRIMOS is a platform specifically designed for use in monitoring of civil structures (Vienna Consulting Engineers, 2008). It has the capability to process and analyze measured response data of a structure and use the data for detection and localization of damage. DEWESoft has developed a software platform for analysis of measured response data from an instrumented structure (DEWESoft, 2007). The platform has the ability to plot and monitor data in a project specific manner, a significant advantage when utilized for monitoring of a network of structures. Strainstall has developed a software platform which can be utilized to evaluate structural parameters such as strain, displacement, force, temperature, inclination, and settlement (Strainstall, 2005). The system can detect change in strain in reinforcement, variation in crack sizes in concrete, vibration in steel girders, or relative movements.

Currently it is common practice to develop a software platform specifically for the structure under consideration, with the requirements and needs of the project built into the software. The Oresund Bridge connecting Denmark and Sweden has been outfitted with a monitoring system which includes a designated software platform for the project (Peeters et al. 2003). A structural health monitoring system has been implemented on The Shandong Binzhou Yellow River Bridge, a cable-stayed bridge in China (Li et al. 2006). Damage detection algorithms based on the modal curvature method, frequency response model, and data fusion technique are incorporated in the platform. As well, member-level information is 
available regarding strain, displacement, and tension forces in the stay cables. The Shenzhen Bay Highway Bridge connecting Shenzhen and Hong Kong has been outfitted with a monitoring system designed with the objective of supporting the management and maintenance of the bridge (Li et al. 2008). Several graphical user interfaces provide access to the monitoring system, including: database maintenance, data collection, 3D model of equipment location and management, video display, traffic statistics, power monitor, pre-warning, and structural safety evaluation. The structural safety interface performs damage identificaiton of the structure via modal identification and finite element analysis. As well, it is used to evaluate the serviceability of the structure and provide a fatigue life estimate of the steel box girder. To assist in the maintenance and management of the structure, weekly, monthly, and annual reports are generated by the system. The Wind and Structural Health Monitoring System (WASHMS) has been implemented on the Tsing Ma Bridge in Hong Kong (Wong, 2003). The system is used to predict the response of the structure to extreme events (Wong, 2003), and predict the service life and fatigue damage of the structure (Chan et al. 2001).

To meet the requirements of processing, analysis, and management of the large volume of data generated from instrumented structures a software platform that is adaptable, scalable, and expandable is desirable. Open software that has the abilities for upgrade and expansion for new data operational functionality would ensure the long-term viability of the software and facilitate the continuous monitoring of a structure. An example of such software is Signal Processing 
Platform for Analysis of Structural Health, which is described in further details in Chapter 3. 


\section{Chapter 3 - Confederation}

\section{Bridge Monitoring Project}

\subsection{Background}

The Confederation Bridge is a prestressed concrete box girder structure, spanning the Northumberland Strait, linking Borden, Prince Edward Island, and Cape Tormentine, New Brunswick in Eastern Canada, as shown in Figure 3-1. At the time of its opening in 1997, it was the world's longest continuous frame bridge over salt water (Cheung et al. 1997), with a total length of $12.9 \mathrm{~km}$. Because of the design requirements of a design service life of 100 years, as compared to the normal 50 to 
75 years for an ordinary highway bridge, its long girder span, and the expected severe conditions at the bridge site, the design of the bridge was not covered by any standard or code. Wind gusts of $100 \mathrm{~km} / \mathrm{hr}$ are typical along the Northumberland Strait, with gusts of $175 \mathrm{~km} / \mathrm{hr}$ recorded during hurricanes along the east coast. The temperature at the bridge site varies from $-40^{\circ} \mathrm{C}$ in the winter to $+35^{\circ} \mathrm{C}$ in the summer. During spring and fall, it is not uncommon for multiple freeze / thaw cycles to occur, which can affect the durability of the concrete structure. The Northumberland Strait is home to strong tidal currents which, during winter, can carry large ice floes, up to $3 \mathrm{~m}$ in depth that can exert significant loads against the piers of the structure. Because of the importance of the structure and the extreme operating conditions it must withstand, more stringent design requirements were adopted in the design of the bridge, including a reliability index of $\beta=4.0$ for multiple load paths and $\beta=4.25$ for single load paths, as compared to $\beta=3.0$ and 3.5 respectively for ordinary highway bridges. A lack of data and information on these load effects resulted in load combinations and resistance factors being formulated specifically for the bridge (MacGregor et al. 1997).

The Confederation Bridge has 21 approach spans, 2 transition spans of length $165 \mathrm{~m}$ each and 43 main spans of $250 \mathrm{~m}$ each. The main section of the bridge is comprised of 22 structural frames, each consisting of a combination of pier base, pier shaft, main girder and drop-in girder connected through post-tensioning, as shown in Figure 3-2. The main girder is comprised of a $250 \mathrm{~m}$ centre span, with two $95 \mathrm{~m}$ overhangs. A continuous frame structure is constructed by post-tensioning of 
Chapter 3 - Confederation Bridge Monitoring Project

the individual structural pieces together. The girder is a prestressed single trapezoidal box girder, varying in depth from $14 \mathrm{~m}$ above the piers to $4.5 \mathrm{~m}$ at midspan, and varying in width from $5 \mathrm{~m}$ at the bottom of the girder to $7 \mathrm{~m}$ at the top.

\subsection{Monitoring System}

In order to gather information that would help to ensure its safe operation and to maintain high performance over its long service life, the Confederation Bridge has been instrumented with a sophisticated monitoring system. The initial objectives of the monitoring system are to verify the design assumptions and parameters used in the bridge design and to obtain information regarding the short and long term performance of the bridge. The data collected by the monitoring system are used to establish a database for structural health monitoring applications and future condition assessment and damage detection of the structure with the goal to minimize its maintenance and repair costs over its service life and to help maintain high service performance. Both bridge response and environmental data regarding wind, traffic, ice, deflection, thermal effects, corrosion and dynamic loadings are recorded. The sensors of the monitoring system are installed on two adjacent spans of the bridge, a drop-in span and a rigid frame.

The environmental loads on the structure are captured by wind sensors, and thermocouples. The bridge is outfitted with two anemometers to capture both wind 
speed and direction at the monitored section. The main objective of the monitoring of thermal effects is to improve the modeling of thermal stresses and further improve prediction models of thermal cracking in a concrete structure. The thermal response of the structure is recorded at seven locations along the structure, as shown in Figure 3-3. Typical cross-sectional layouts of thermocouples are shown in Figure 3-4 and Figure 3-5. Three pyranometers are installed with the monitoring system to measure solar radiation, along with thermocouples to measure temperature distribution both inside and outside of the box girder. Along with the thermocouples, vibrating wire strain gauges are installed, as shown in Figure 3-6, to monitor strain distribution in the bridge members.

Due to the significant ice forces exerted on the pier structure of the bridge, the monitoring system includes instrumentation specifically designed for the verification of the design ice loads used during its design. The ice loads are monitored by tiltmeters which record the tilt response of the pier. Along with the tiltmeters, four video cameras are installed to capture the interaction of the ice floes with the piers and two sonars have been deployed to measure the depth of the ice floes.

Due to the exposure of the bridge to salt water, its corrosion performance is monitored to ensure its long term durability and the optimum performance of the structure. Corrosion probes have been installed at the splash zones of the bridge piers and near expansion joints of the instrumented frame section of the bridge. 
To monitor the short and long term displacement and deformation behaviour of the structure, the strength and modulus of elasticity of the concrete material of the bridge is monitored by measurements obtained from eighteen concrete cylinders from three casts. These measurements are compared to the assumed values used in the bridge design. In addition, three concrete casts from the bridge are monitored for their creep and shrinkage properties. The monitoring data are compared with the values used in the design of the bridge. The prestressing loss is measured in four tests on three samples of prestressing steel. The displacements of the structure are monitored in both the vertical and horizontal directions. The vertical displacements are measured at seventeen locations inside the girder, while horizontal displacements are measured at four points at eleven cross sections, as shown in Figure 3-7. Displacements at the expansion joints between piers 30 and 31 , and 32 and 33 are monitored by eight displacement transducers.

The dynamic responses of the structure, due to the effects of wind, traffic, ice floe, and earthquake loadings are captured by 62 accelerometers distributed over the instrumented section of the bridge, as shown in Figure 3-8 and Table 3-1. The response is captured in both the vertical and horizontal directions, which allows the system to capture vertical bending, transverse bending, and torsional vibration modes. An automatic continuous data collection system has been developed to collect and store the dynamic data. If a predetermined threshold is exceeded, a ten minute data set is recorded, including a thirty second pre-triggering buffer, at a sampling rate of $125 \mathrm{~Hz}$. Otherwise simply statistical data, such as mean and 
standard deviation, are stored to allow for the establishment of long term trends regarding the performance of the structure.

Data are collected by a series of data loggers, which transmit the data to on shore computers. Data are then stored in a central computer on shore. There the data are transmitted through the internet to computing facilities of the OttawaCarleton Bridge Research Institute in Ottawa for real-time data processing and evaluation or for data archival. Figure 3-9 shows the flow of data from sensors to the Ottawa-Carleton Bridge Research Institute at Carleton University.

\subsection{Signal Processing Platform for Analysis of}

\section{Structural Health}

In order to minimize the effort required for processing of large volume of data collected by the Confederation Bridge monitoring project, a robust modular software system has been developed for the project. The use of this software, Signal Processing Platform for Analysis of Structural Health (SPPLASH), overcomes the common difficulties encountered in electronic monitoring of structures by employing advanced system identification techniques to extract the desired engineering information from the data in a timely fashion. The software has been developed in a modular fashion to allow for the incorporation of new technologies 
and techniques as they become available in the future. SPPLASH is also designed to have a structure independent framework, thus allowing for the use of the software in other monitoring projects, or in a network of bridges. The main aspects of the software are described herein. A more in-depth description can be found in the reference Desjardins (2004).

\subsubsection{Data Processing}

Data processing is necessary for a monitoring system such as the one discussed on the Confederation Bridge. The processing step prepares the data in the proper format and checks for their validity for analysis prior to extraction and interpretation of engineering information. Typical issues which must be resolved for data collected from an instrumented structure are:

- As a result of the data being recorded by multiple data loggers, a lack of synchronization of data time-stamps is possible.

- Small gaps may be present in the data due to the high sampling rate necessary to capture all information. If the CPU of the data logger is overworked during the recording of an event, gaps may occur.

- Duplication of data samples is possible due to an error in the acquisition software.

- An offset may be present in the data. 
Chapter 3 -Confederation Bridge Monitoring Project

- High frequency noise may be present in the data due to electrical noise contaminations.

- When using multiple data loggers, the sampling rate amongst the loggers may not be constant.

- Data from a particular event may be separated into different files if an event occurs during a prescheduled automatic collection of the data from a logger.

- A single data file may contain multiple events.

SPPLASH has been developed with the necessary algorithms to correct the aforementioned errors. The correction of these errors has been subdivided into two categories, pre-processing and processing. The pre-processing algorithms are used solely for the management of the data, i.e. no changes are made to the data samples. Whereas the processing algorithms may adjust the data samples as necessary.

The pre-processing of data is achieved through the following processes:

- Complete datasets are extracted from the necessary data files to form a single dataset.

- Data files are converted to the necessary format for further processing and analysis.

- Complete data events are assembled from all data loggers, where a data event is considered the assembly of a dataset from involved data logger.

Processing of data comprises of the following steps: 
- Small gaps that may be present in the data are filled through the use of a Piecewise Cubic Hermit Interpolating Polynomial, using four samples before and after the gap.

- Data records with a sampling interval less than zero are compared with previous records to determine if duplication is present, if so the record is removed from the data file.

- The baseline offset of a sensor is removed by determining the mean of the signal and purging it from the record, as shown in Figure 3-10.

- Data samples are converted from measured voltage readings to engineering unit of $\mathrm{m} / \mathrm{s}^{2}$.

- As the data event consists of datasets from multiple data loggers, a consistent sampling rate is employed. The minimum sampling rate from the data loggers under consideration is determined, and all loggers with a higher rate are down-sampled. A finite impulse response filter is utilized to prevent aliasing.

- A typical sampling rate for dynamic data is $125 \mathrm{~Hz}$, whereas in some analysis if the frequency of interest of the structure is commonly below $15 \mathrm{~Hz}$, computational loads can be reduced through decimation of the data. In order to prevent aliasing during this process a low-pass Chebyshev type I filter is employed. 
- The acceleration data is doubly integrated, through cumulative trapezoidal numerical integration, to obtain displacement data of the structure, which are utilized in the visualization module.

All of the aforementioned pre-processing and processing algorithms are employed in a single graphical user interface, as shown in Figure 3-11, which provides the user with updates on the progress of the tasks.

\subsubsection{Data Analysis}

Following the processing of monitoring data, analysis tools are utilized to extract relevant engineering information of the monitored structure. SPPLASH has the capability to determine the power spectral density (PSD) of a sensor based on Welch's method (Welch, 1967). The PSD of an individual sensor can provide insights into the operational status of the sensor. An example of a PSD of an operational sensor is shown in Figure 3-12. PSD can be utilized to determine the dominant structural frequencies in the data; however for more accurate and thorough analysis more sophisticated techniques are necessary.

An important component of intelligent monitoring of structures is the determination of modal properties of a structure, such as vibration frequencies, damping ratios, and mode shapes. In order to extract this information from the monitoring data the stochastic subspace identification technique has been 
Chapter 3 -Confederation Bridge Monitoring Project

implemented in SPPLASH. A common aspect of many system identification techniques is the use of a stabilization diagram to determine the physical poles present in data. SPPLASH has been incorporated with a GUI which allows for user selection of the poles of the system, as shown in Figure 3-13. A thorough description of stochastic subspace identification is provided in Chapter 4.

\subsubsection{Data Animation and Visualization}

To facilitate the interpretation of the extracted information from the monitoring data, SPPLASH has incorporated a visualization module which can provide the user with near real-time display of the responses of the structure to loadings. The module features a 3D model representation of the structure which displays the response of the structure at the model nodes through interpolation of the response at sensor locations.

Along with displacements of the structure, experimental and analytical mode-shapes can be visualized through use of the GUl. This ability can provide significant clarity when attempting to distinguish between vibration modes of similar frequencies. The module allows for animation of current data events, or of those extracted from a database.

Figure 3-14 shows the animation of the monitored section of the Confederation Bridge. 
Table 3-1 : Accelerometer locations and channels for Confederation Bridge monitoring project

\begin{tabular}{|c|c|c|c|c|c|c|c|c|c|}
\hline \multirow{2}{*}{ Pier } & \multirow{2}{*}{ Logger } & \multirow{2}{*}{ Location } & \multicolumn{5}{|c|}{ Channel } & \multirow{2}{*}{$\begin{array}{c}\text { Scale } \\
\mathrm{v} / \mathrm{g}\end{array}$} & \multirow{2}{*}{ Model } \\
\hline & & & $T_{1}$ & $T_{2}$ & $L$ & $V_{1}$ & $v_{2}$ & & \\
\hline \multirow{5}{*}{ P30 } & \multirow{5}{*}{1} & 1 & 7 & & 8 & 9 & & 1 & PCB 326 A03 \\
\hline & & 3 & 12 & & 13 & & & 2.66 & QLC 400 \\
\hline & & 4 & 10 & & 11 & & & 2.66 & QLC 400 \\
\hline & & 16 & & 3 & & 1 & 2 & 1 & PCB 393C \\
\hline & & 17 & & 6 & & 4 & 5 & 1 & PCB 393C \\
\hline \multirow{11}{*}{ P31 } & \multirow{11}{*}{4} & 1 & & & & & & 1 & PCB 326 A03 \\
\hline & & 2 & & & & & & 1 & PCB 326 A03 \\
\hline & & 3 & 1 & & 2 & & & 99 & QA 1200 \\
\hline & & 4 & 3 & & 4 & & & 99 & QA 1200 \\
\hline & & 10 & & 23 & & 22 & 24 & 1 & PCB 393C \\
\hline & & 11 & & 19 & & 21 & 20 & 2.66 & QLC 400 \\
\hline & & 12 & & 8 & & 9 & 7 & 1 & PCB 393C \\
\hline & & 13 & & 10 & & 12 & 11 & 2.66 & QLC 400 \\
\hline & & 14 & & 13 & & 14 & 15 & 1 & PCB 393C \\
\hline & & 15 & & & & 18 & 17 & 2.66 & QLC 400 \\
\hline & & 18 & 5 & & 6 & 16 & & 99 & QA 1200 \\
\hline \multirow{9}{*}{ P32 } & \multirow{9}{*}{5} & 1 & 1 & & 2 & 3 & & 1 & PCB 326 A03 \\
\hline & & 2 & 4 & & 5 & 6 & & 1 & PCB 326 A03 \\
\hline & & 3 & 10 & & 11 & & & 99 & QA 1200 \\
\hline & & 4 & 16 & & 15 & & & 99 & QA 1200 \\
\hline & & 5 & 24 & & & 23 & 26 & 1 & PCB 393C \\
\hline & & 6 & 28 & & & 26 & 27 & 1 & PCB 393C \\
\hline & & 7 & 30 & & & 29 & 31 & 1 & PCB 393C \\
\hline & & 8 & 32 & & & 34 & 33 & 1 & PCB 393C \\
\hline & & 9 & 20 & & & 21 & 22 & 2.66 & QLC 1200 \\
\hline
\end{tabular}


จ

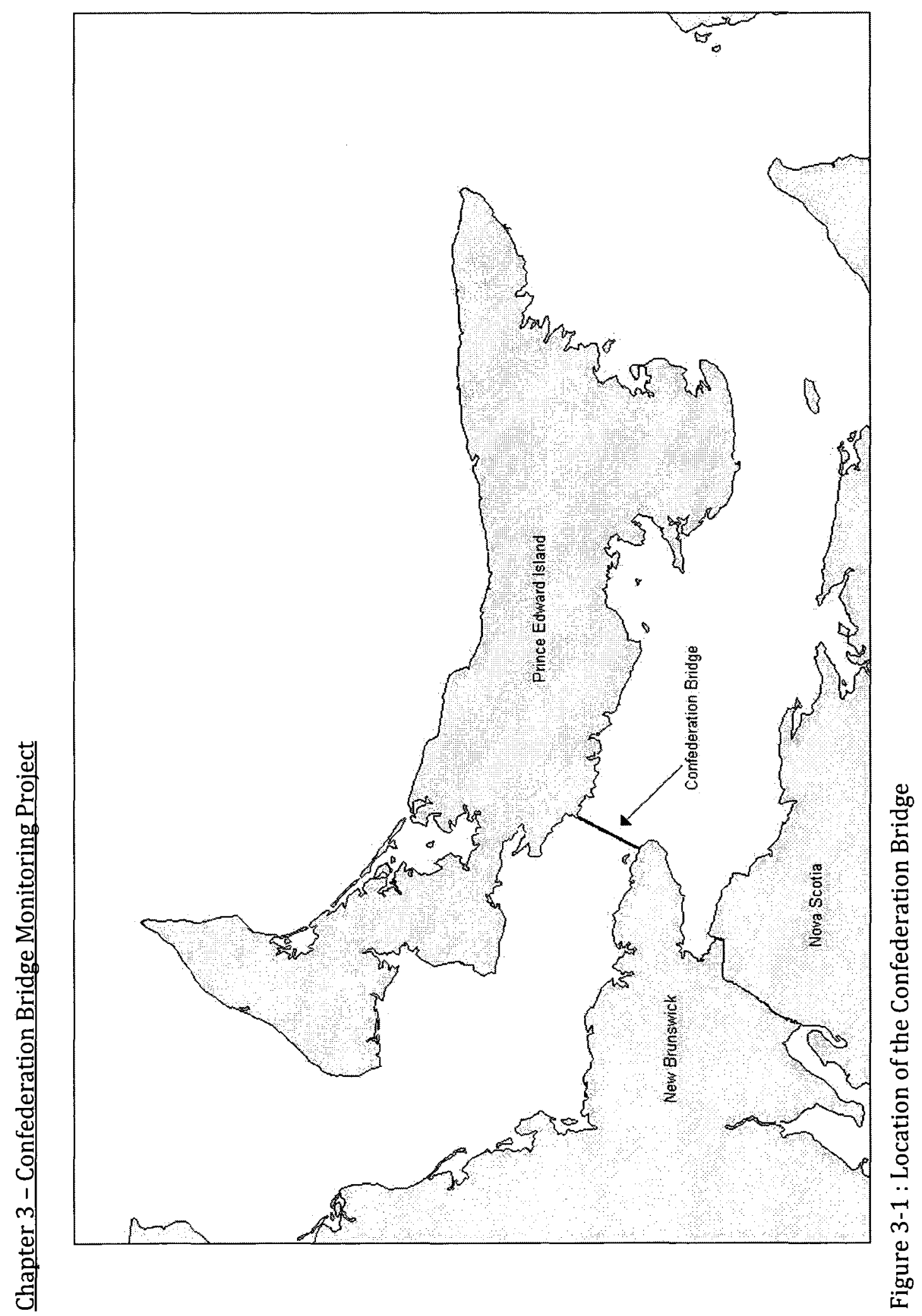


요

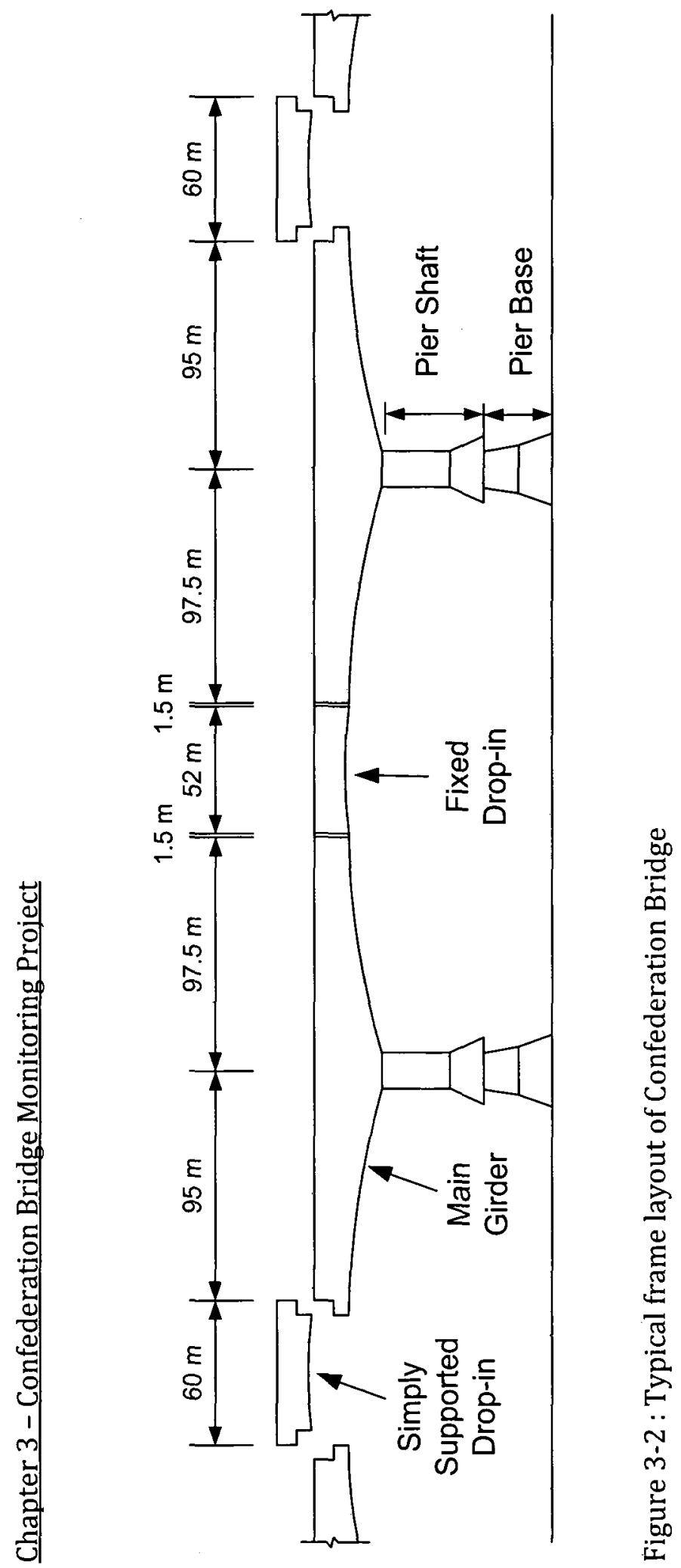




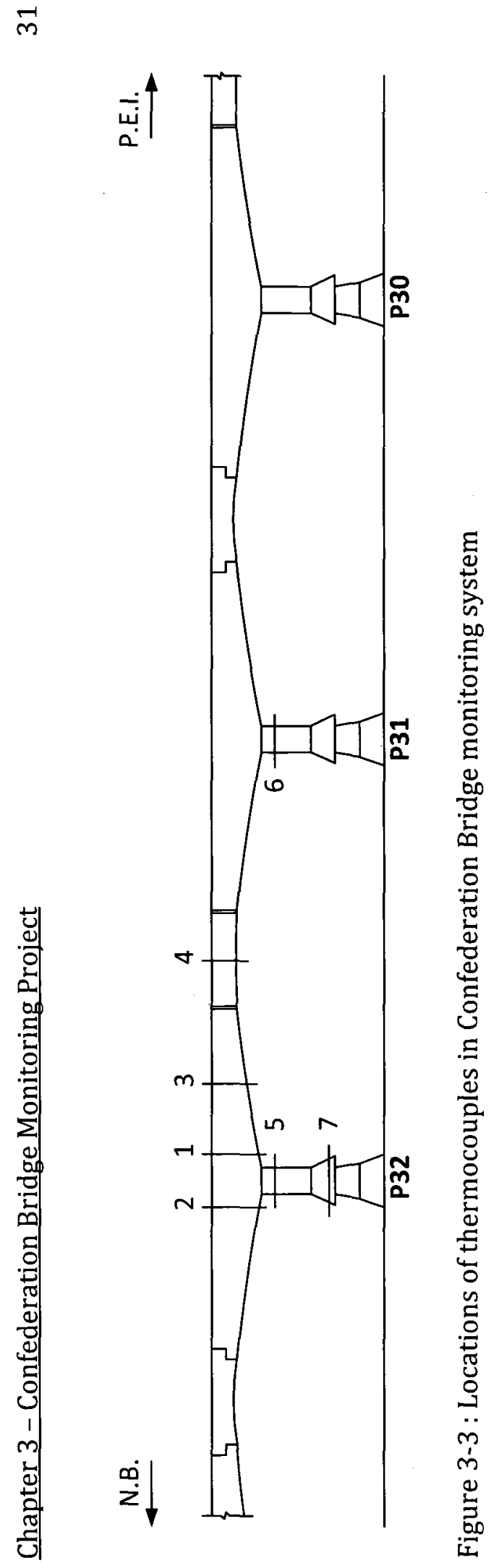




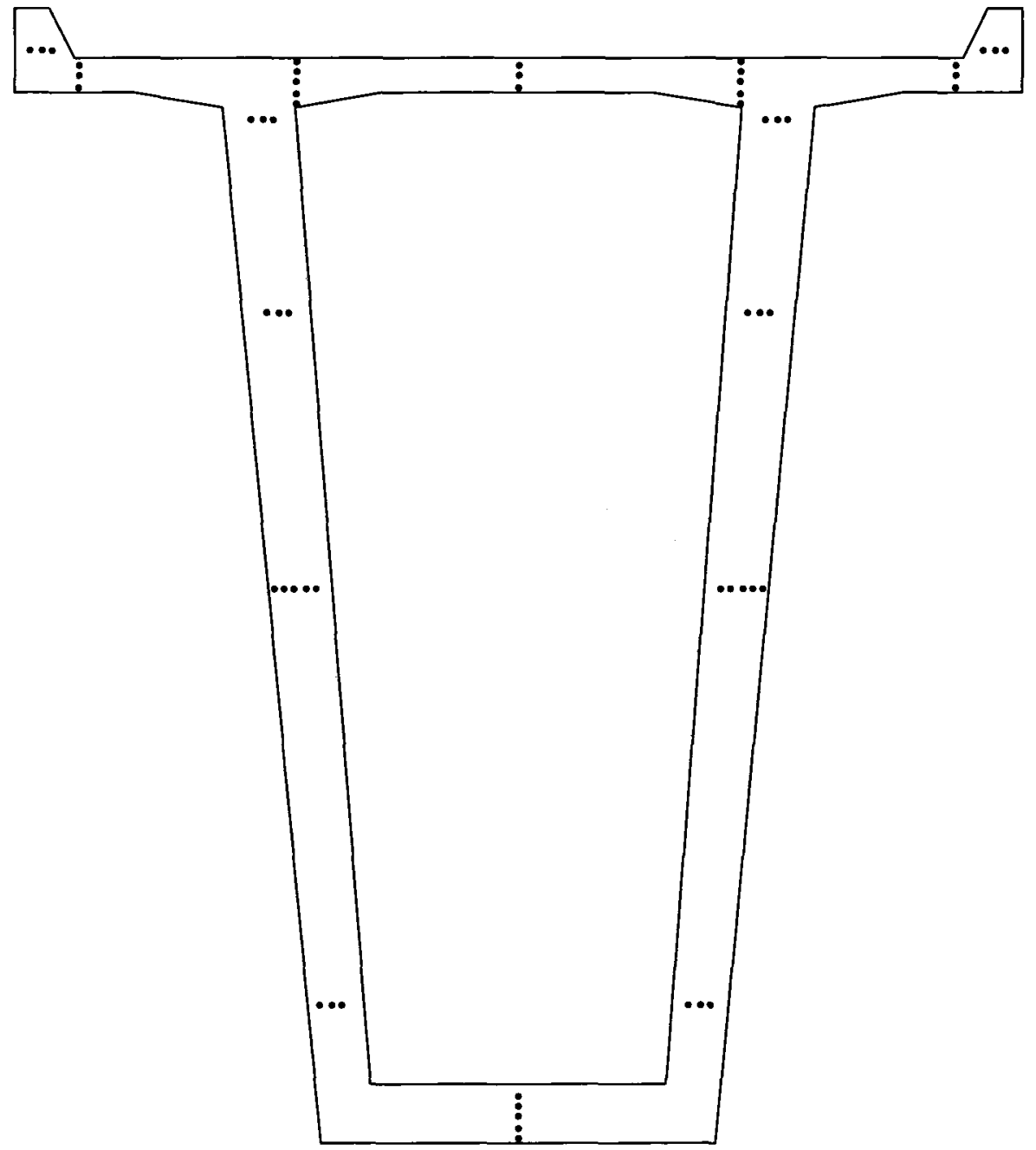

Figure 3-4 : Typical thermocouple layout of girder in Confederation Bridge monitoring system 

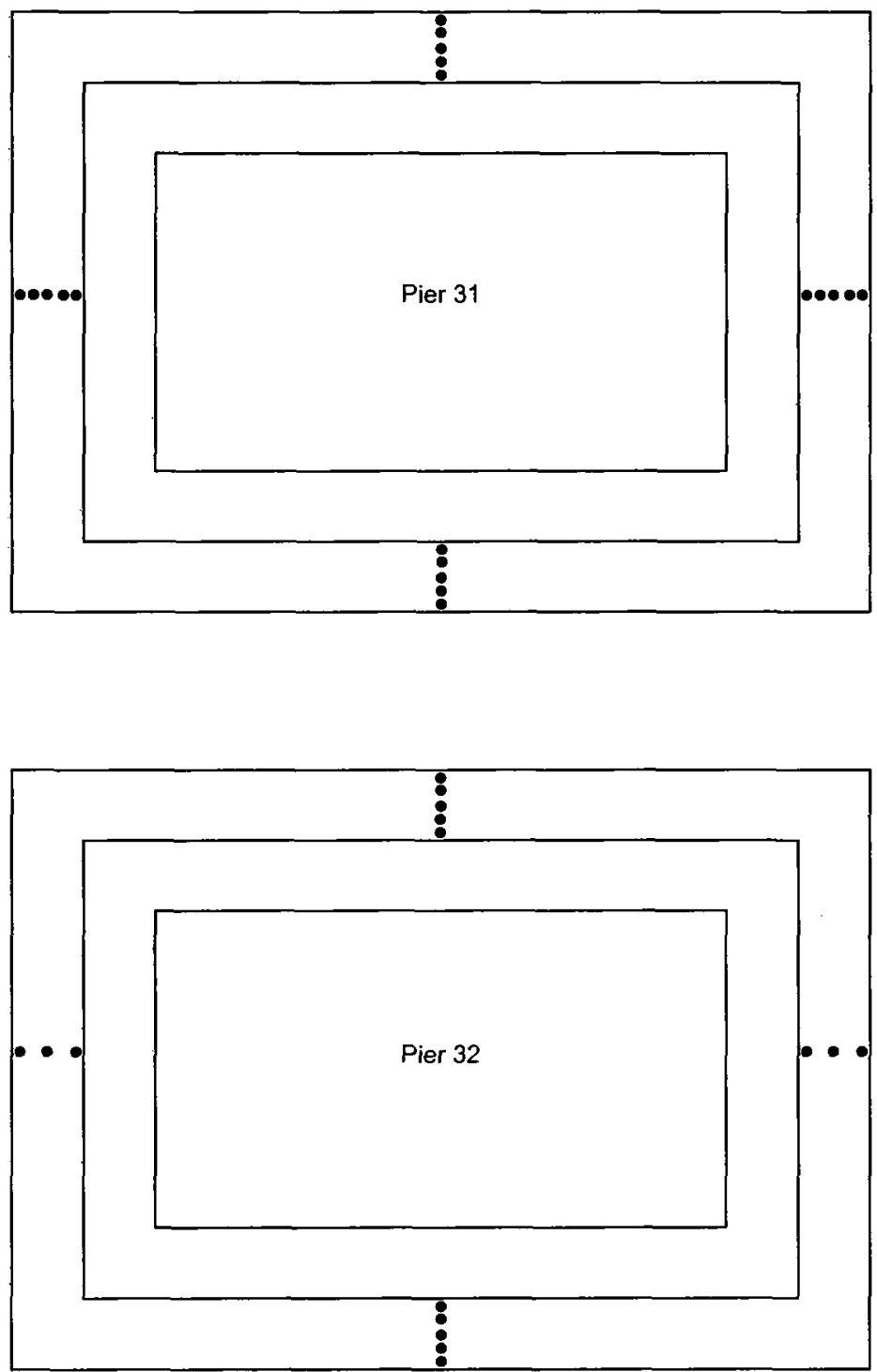

Figure 3-5 : Thermocouple layout of pier in Confederation Bridge monitoring system 


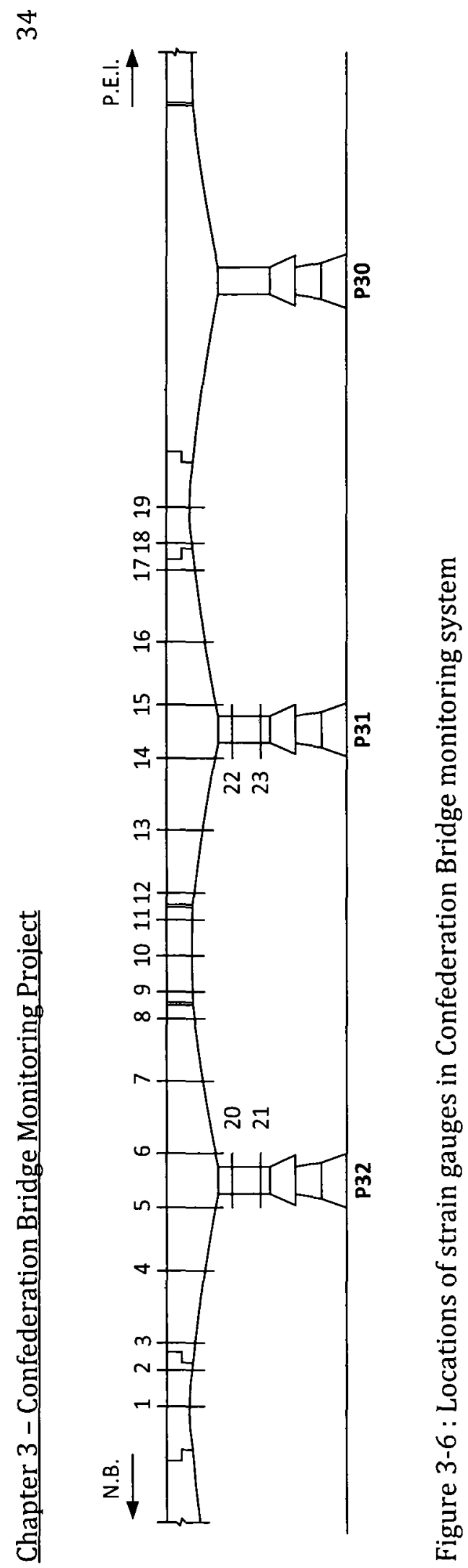




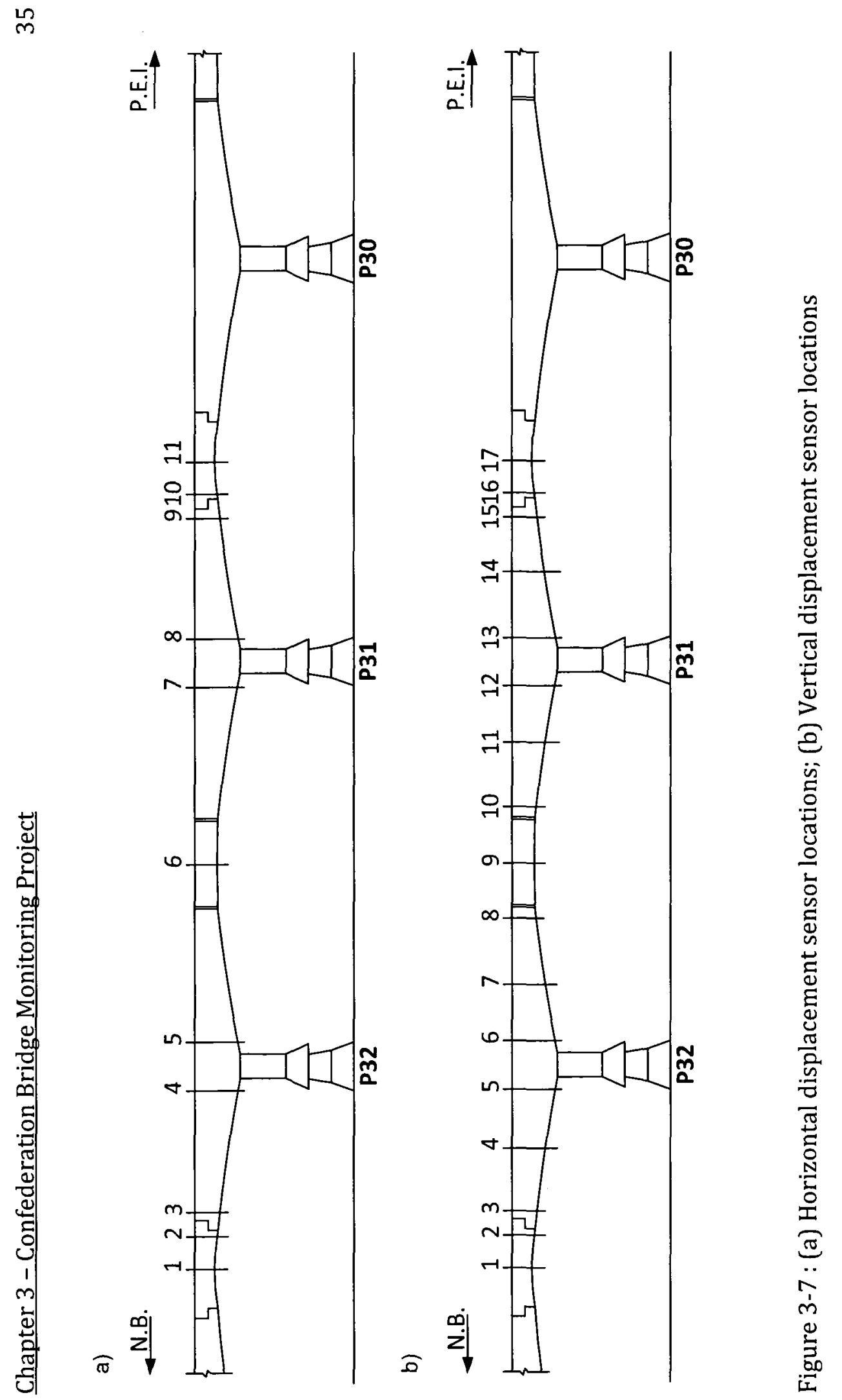




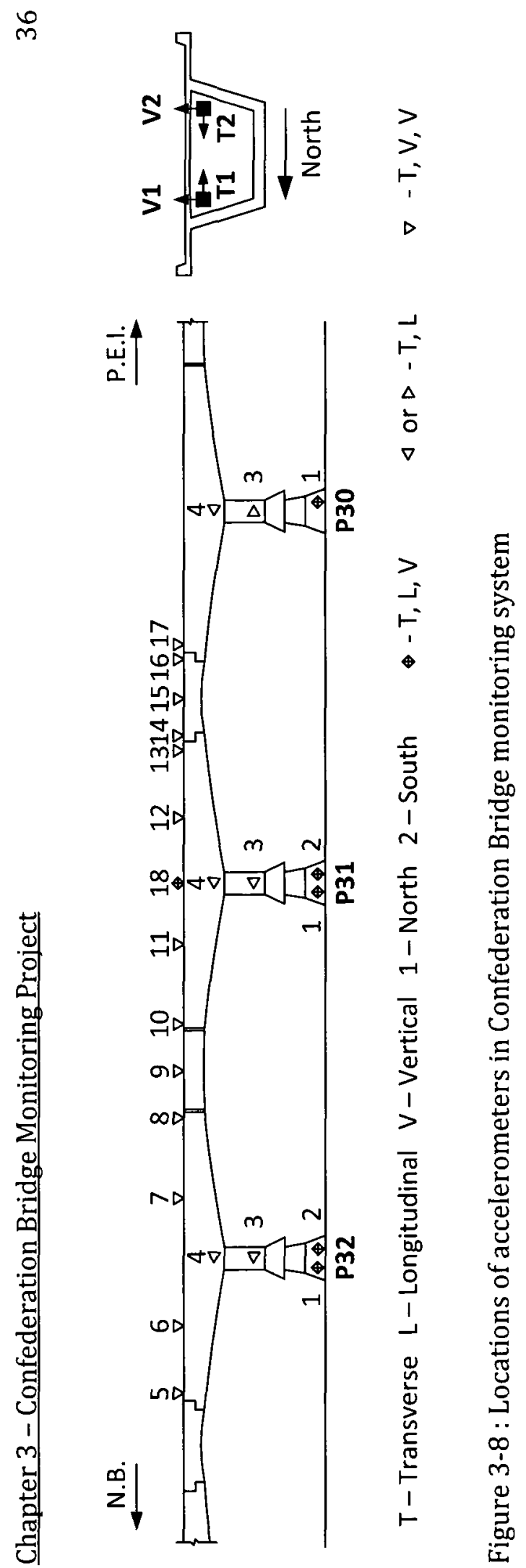




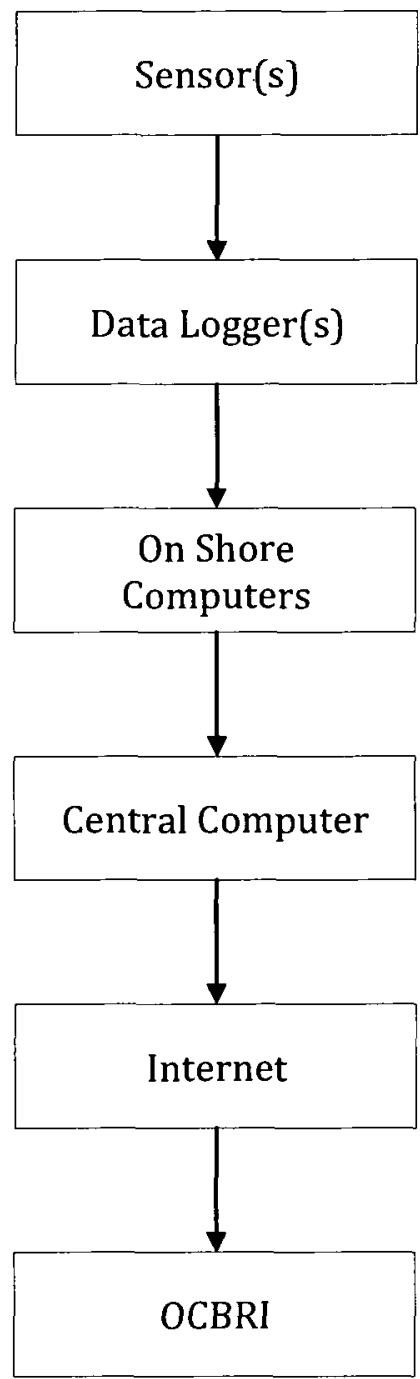

Figure 3-9 : Data flow through Confederation Bridge monitoring system 
Chapter 3 - Confederation Bridge Monitoring Project

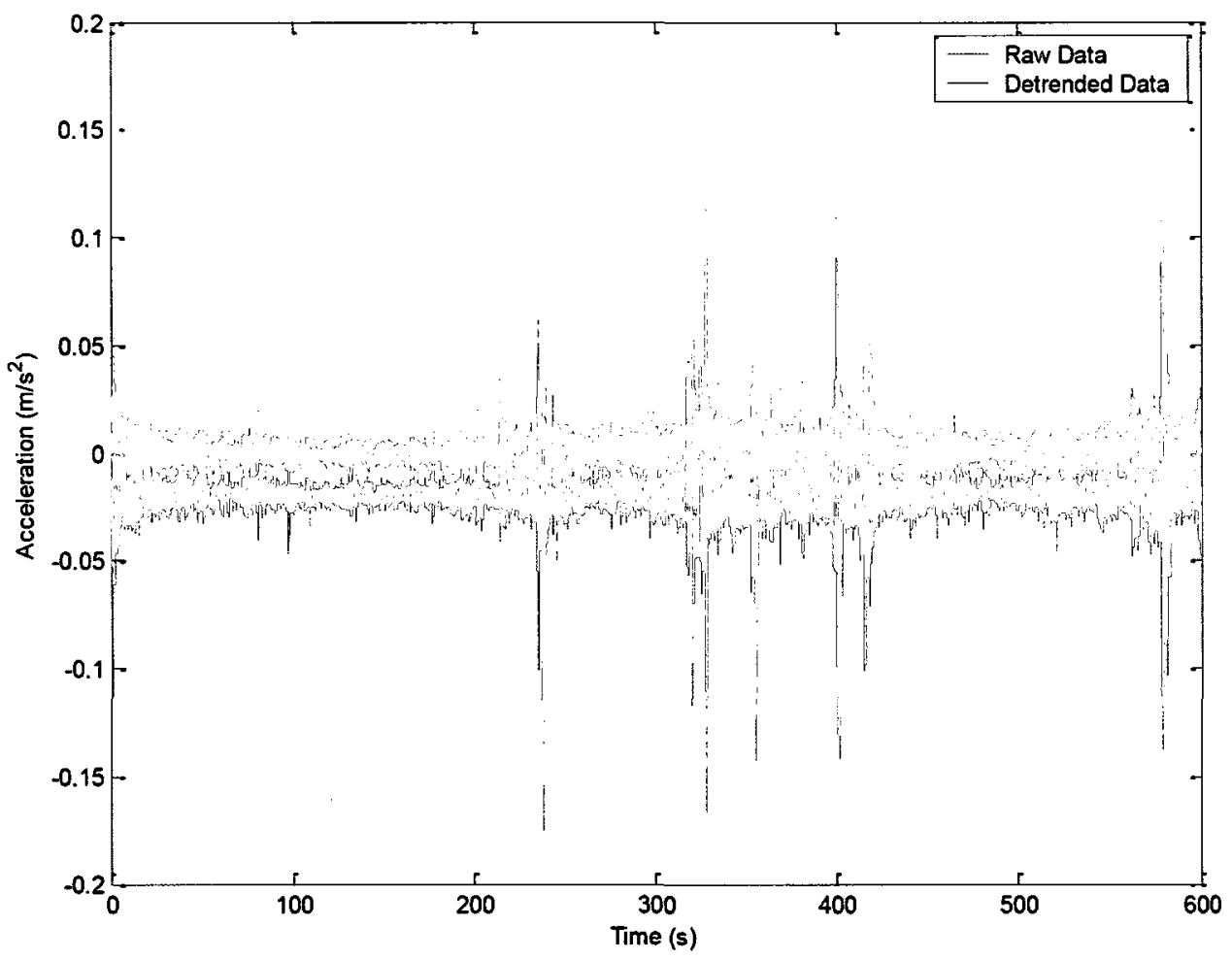

Figure 3-10: Removal of baseline offset from measured response data 


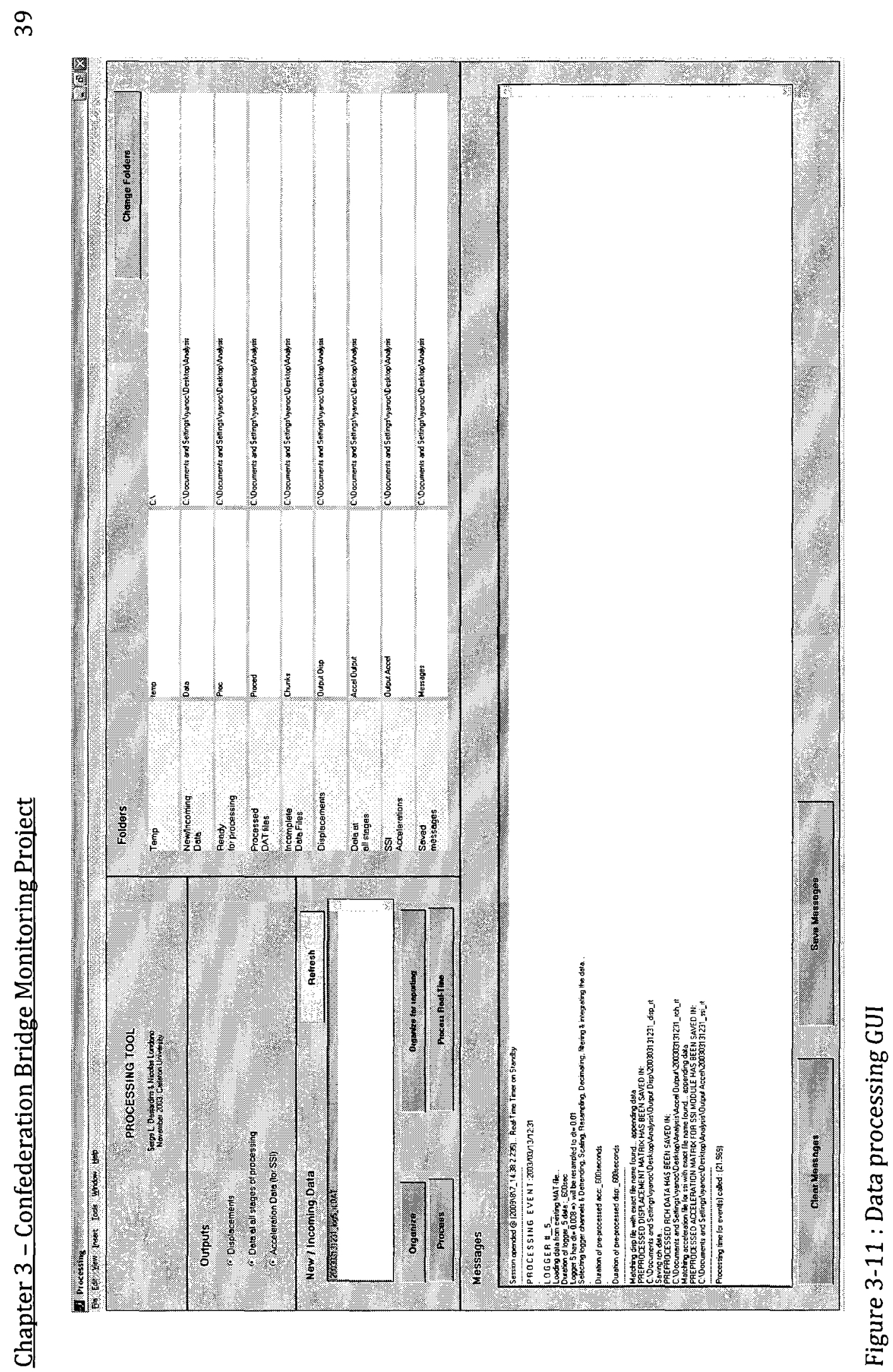




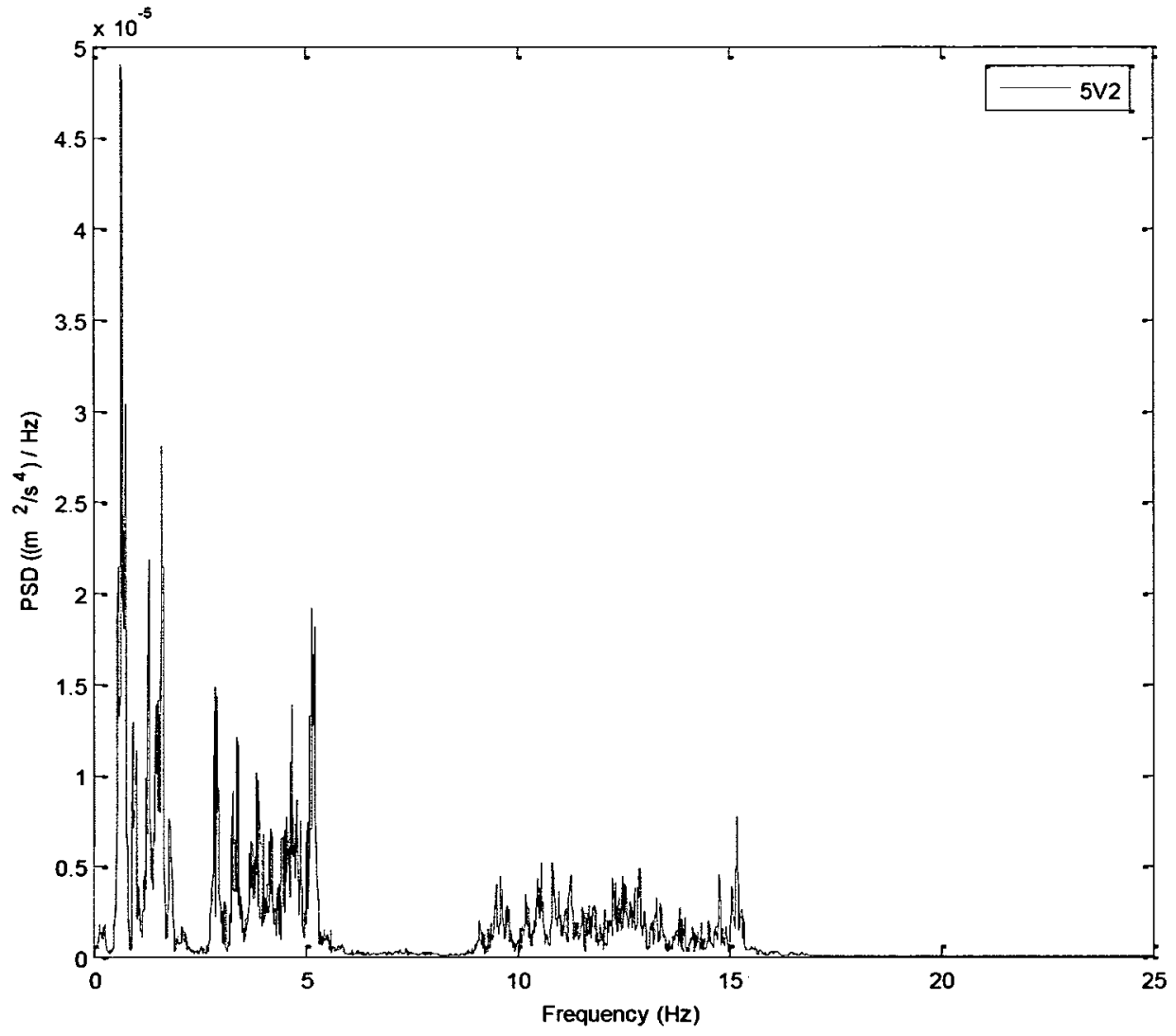

Figure 3-12 : Power spectral density of a single sensor 


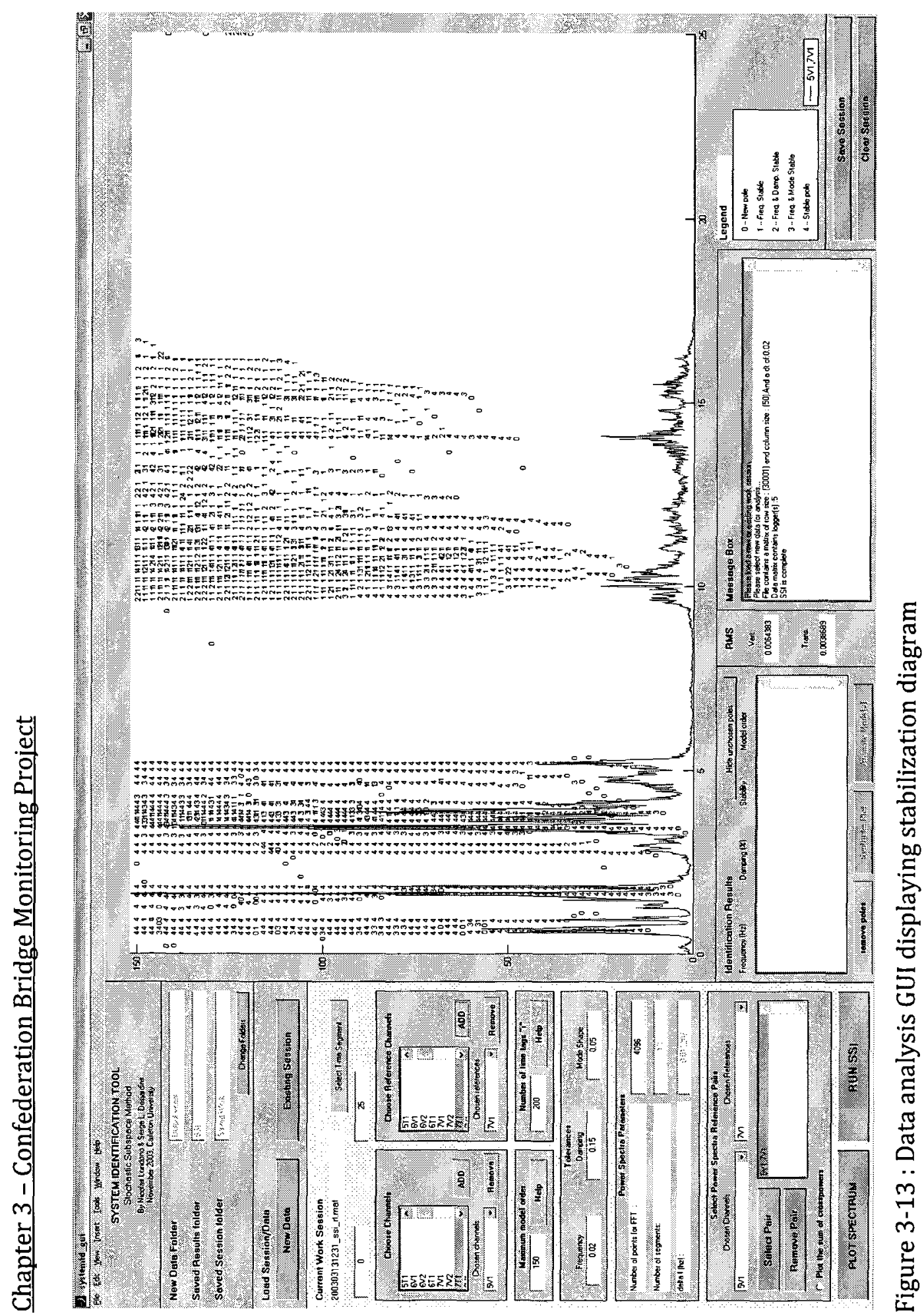


ร

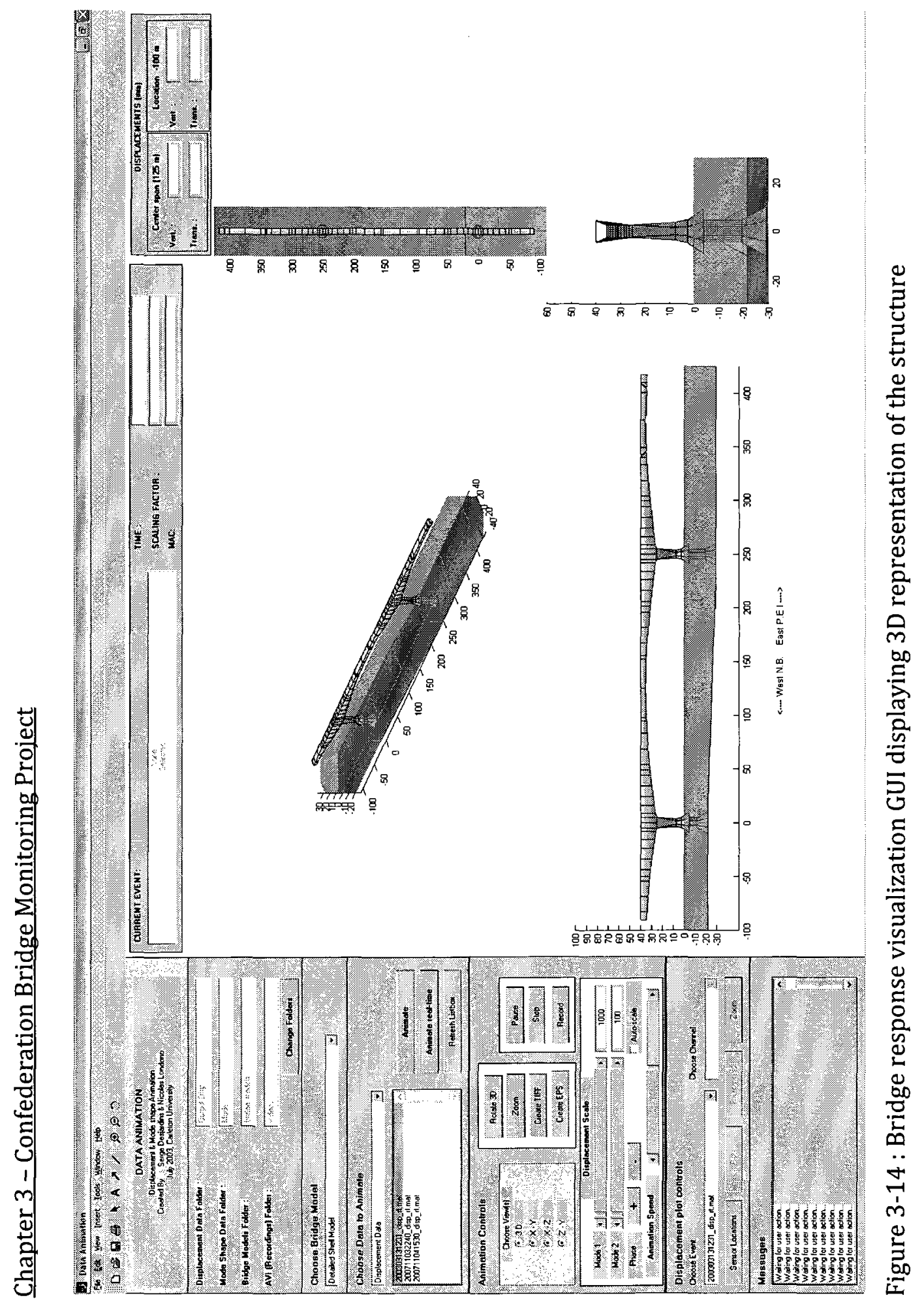




\section{Chapter 4 - Vibration Based}

\section{Structural Health Monitoring}

\subsection{Introduction}

Bridges are an integral part of today's infrastructure, which require regular maintenance and periodic repair to ensure high service level and performance in providing service to the public. To develop the most cost effective maintenance and repair strategies, information regarding the current condition and expected deterioration of a structure are needed. To determine the current condition of a structure one of several monitoring techniques can be employed. The predominant 
practice of obtaining information about the condition of a bridge is manual visual inspection, which may be aided by other non-destructive localized techniques such as acoustic emission, ultrasonic methods, or the tap test. However the ability of these methods to determine an accurate measure of a structure's condition is hindered by several limitations, such as inability to detect internal damage, inaccessibility to certain areas of the structure, the need of a priori knowledge of the area of interest. Another critical limitation associated with the aforementioned techniques is their periodic nature, which does not allow for the determination of a useful rate of change in the condition of a structure, unless they are performed at a high frequency, which is an unrealistic expectation due to cost restraint. Therefore it is desirable to develop new innovative techniques, which can provide more accurate and more detailed results, such as continuous monitoring of a structure.

Recently, the technology of vibration based structural health monitoring (VBSHM) has garnered significant attention as an effective alternative approach that can lead to consistent and reliable methods for continuous monitoring of structures. VBSHM is based on the basic premise that vibration properties of a structure are dependent on the physical characteristics (mass, damping, stiffness) of a structure. As a result, any change in the vibration properties of a structure can be linked to a variation in the physical characteristics or conditions of a structure. Under the assumption that these physical characteristics remain constant over time, any variation or detection of change in these physical characteristics can thus be attributed to deterioration or damage in the structure. In order to determine the 
actual field vibration properties of a structure, system identification techniques must be utilized on the measured vibration response data of the structure.

It is common practice to evaluate mechanical systems through the use of forced vibration measurements. Due to their size and complexity, it is impractical to obtain the vibration properties of large civil structures, such as the Confederation Bridge, using the same technique. Additionally, forced vibration tests can only be carried out at periodic times in the structure's service life. In order to continuously monitor a structure, methods which rely upon its responses to ambient excitations such as wind and traffic loadings are necessary. Collection of ambient vibration response data in a continuous monitoring system generates large volume of data. These captured ambient vibration response monitoring data are used in the calibration of numerical computer models of the real-life structure. The calibrated computer model of the structure thus contains the same structural condition information as the monitoring data. This process is known as system identification. An output-only system identification technique which requires no knowledge of the input forces is utilized to determine the actual modal vibration properties of the structure from the measured ambient vibration response data. There are several different output-only system identification techniques available for monitoring of civil engineering systems, such as peak-picking, rational fraction polynomial, lbrahim time-domain method, eigensystem realization algorithm, and stochastic subspace identification. In a comparison study, Peeters \& Ventura (2003) show that that stochastic subspace identification yields the most consistent and reliable 
results in the extracted modal vibration properties from ambient vibration response measurement data. Recently a new frequency-domain technique has been developed, the polyreference least squares complex frequency method (Peeters et al. 2004a). These two techniques are adapted and implemented in this study to analyze the vibration monitoring data obtained from the Confederation Bridge monitoring project.

\subsection{Peak Picking}

One of the basic methods for estimating the modal parameters of a dynamic system is the peak-picking method, which derives its name from its key component in the identification of modal frequencies as peaks in the power spectral density plot. The power spectral density (PSD) function is the power content of a signal in a frequency band. A modified version of the PSD has been developed by Welch (1967), which divides the discrete-time signal data into overlapping windowed segments of equal lengths. Windowing is used to reduce the effects of leakage when performing the Fourier transform to convert the data from the time domain to the frequency domain. A common window used for determination of the PSD is the Hamming window, as seen in Figure 4-1, which is defined as follows (Marple, 1987)

$$
w(n)=0.54+0.46 \cos (2 \pi t(n))
$$

where $\mathbf{t}(\mathbf{n})$ is defined as 


$$
\mathbf{t}(\mathbf{n})=\frac{\left(\mathbf{n}-\frac{\mathbf{N}-\mathbf{1}}{2}\right)}{\mathbf{n}-\mathbf{1}}
$$

where $\mathbf{n}$ is the current sample and $\mathbf{N}$ is the total number of samples. The effect of the hamming window on a segment of data is clearly evident as shown in Figure 4-2.

The periodogram of the windowed segment is then calculated as the Discrete Fourier Transform of the data:

$$
X_{h}(\omega)=\sum_{n=0}^{N-1} w(n) y(n) e^{-j \omega n T_{s}}
$$

where $\mathbf{y}(\mathbf{n})$ is the measured data and $\mathbf{T}_{\mathbf{s}}$ is the sampling period.

The PSD is calculated as the average of the periodograms:

$$
\operatorname{PSD}_{\mathrm{i}}(\omega)=\frac{1}{M} \sum_{\mathrm{h}=1}^{\mathrm{M}} \mathrm{X}_{\mathrm{h}}(\omega)
$$

where $\mathbf{M}$ is the number of periodograms.

It has been shown that the use of the PSD of sensor measurement data at a single location is not adequate to identify all of the natural frequencies that may be significant of the dynamic system. To overcome this problem, the average normalized power spectral density (ANPSD) is introduced (Felber, 1993). The normalized power spectral density is calculated as follows:

$$
\operatorname{NPSD}_{i}\left(\omega_{k}\right)=\frac{\operatorname{PSD}_{i}\left(\omega_{k}\right)}{\sum_{k=0}^{N_{f}} \operatorname{PSD}_{i}\left(\omega_{k}\right)}
$$

The ANPSD can be determined based on the number of channels, $\mathbf{l}$, present in the data. 


$$
\operatorname{ANPSD}\left(\omega_{k}\right)=\frac{1}{1} \sum_{i=1}^{1} \operatorname{NPSD}_{i}\left(\omega_{k}\right)
$$

There are several drawbacks to peak picking, such as the inability to determine closely spaced modal frequencies, lack of damping estimates, and subjectivity due to analyst identification of the modal frequencies. Despite these shortcomings, observations from an ANPSD plot still provide valuable insights on the behaviour and characteristics of a dynamic system.

\subsection{Stochastic Subspace Identification}

A reliable method of system identification for operational modal analysis of ambient vibration response data is stochastic subspace identification (Londono, 2006), (Peeters \& Ventura, 2003). This technique is outlined herein. A more indepth explanation of SSI can be found in the reference Peeters (2000).

The dynamic behaviour of a system can be modeled by the equation of motion as follows:

$$
\mathbf{m} \ddot{\mathbf{u}}(\mathrm{t})+\mathbf{c u}(\mathrm{t})+\mathbf{k u}(\mathrm{t})=\mathbf{f}(\mathrm{t})
$$

where $\mathbf{u}$ is an $n \times 1$ displacement vector, $\mathbf{m}, \mathbf{c}$, and $\mathbf{k}$ are $n \times n$ mass, damping, and stiffness matrices respectively, and $\mathbf{f}$ is the $n \times 1$ load vector of an $n$ degree-offreedom model. To model the dynamic behaviour of a real-life structure, with its 
response sampled at discrete intervals, a discrete-time state-space model is often used

$$
\begin{aligned}
& x_{k+1}=A x_{k}+B p_{k} \\
& y_{k}=C x_{k}+D p_{k}
\end{aligned}
$$

where $\mathbf{x}_{\mathbf{k}}$ is a $2 n \times 1$ state vector which describes the system displacement and velocity at the instant $k \Delta t$, where $\Delta t$ is the sampling interval, $\mathbf{A}$ is the discrete state matrix, $\mathbf{B}$ is the discrete input matrix, $\mathbf{p}_{\mathbf{k}}$ is an $m \times 1$ sampled excitation vector, where $m$ is the number of inputs of the system, $y_{k}$ is an $l \times 1$ measured output vector, where $l$ is the number of outputs of the system, $\mathbf{C}$ is the discrete output matrix, and D is the direct transmission matrix. The discrete-time state-space model in Equation (4-8) is a deterministic formulation, requiring complete knowledge of the input values. For an output-only system the sampled excitation vector $\mathbf{p}_{\mathbf{k}}$ is not measured due to practical reasons. Therefore, the model formulation is adapted to a stochastic model. To accomplish this the input terms $\mathbf{B} \mathbf{p}_{\mathbf{k}}$ and $\mathbf{D} \mathbf{p}_{\mathbf{k}}$ are replaced with stochastic white noise terms. The discrete-time state-space model can now be written as follows:

$$
\begin{aligned}
& x_{k+1}=A x_{k}+w_{k} \\
& y_{k}=C x_{k}+v_{k}
\end{aligned}
$$

where $\mathbf{w}_{\mathbf{k}}$ and $\mathbf{v}_{\mathbf{k}}$ are white, zero-mean stochastic processes, independent of the state vector. The measured inputs can be displacements, velocities, or accelerations, which are the measured inputs of the Confederation Bridge. 
Assuming stationarity of the state vector and whiteness of the input terms, it can be shown that the correlations of the outputs, $\mathbf{R}_{\mathrm{i}}$, can be factorized into a triplet containing the state matrices (Peeters, 2000).

$$
\mathbf{R}_{\mathbf{i}}=\mathrm{CA}^{\mathrm{i}-\mathbf{1}} \mathbf{G}
$$

where $\mathbf{R}_{\mathbf{i}}$ is defined as $\mathbf{R}=\mathbf{E}\left[\mathbf{y}_{\mathbf{k}+\mathbf{i}} \mathbf{y}_{\mathbf{k}}^{\mathrm{T}}\right]$, with $\mathbf{E}$ denoting the expectation operator, and $\mathbf{G}=\mathbf{E}\left[\mathbf{x}_{\mathbf{k}+\mathbf{1}} \mathbf{y}_{\mathbf{k}}^{\mathrm{T}}\right]$. The basis of SSI can be derived from Equation (4-10), which demonstrates that decomposition of the correlations yields the state-space matrices, which contain the structural parameters of the system.

The correlations can be estimated from the data as follows:

$$
R_{i}=\frac{1}{N} \sum_{k=0}^{N-1} y_{k+\mathrm{i}} \mathrm{y}_{\mathrm{k}}^{\mathrm{T}}
$$

where $\mathbf{y}_{\mathbf{k}}$ is a column vector of measured response at the instant $k \Delta t$ and $\mathbf{N}$ is the number of samples. These correlations significantly compress the data while maintaining the crucial modal information.

In operational modal analysis, data from multiple sensors are used to obtain accurate mode shapes. As all the sensors contain essentially the same information with respect to frequency and damping ratios, there is often redundancy in the data. Due to this, the computational requirements can be greatly reduced by calculating the data correlations with respect to a small set of reference sensors. A block Toeplitz matrix of data correlations at different time lags is then assembled as follows: 


$$
\mathbf{T}_{1 \mid \mathbf{i}}^{\mathrm{ref}}=\left[\begin{array}{cccc}
\mathbf{R}_{\mathbf{i}}^{\text {ref }} & \mathbf{R}_{\mathbf{i}-1}^{\text {ref }} & \cdots & \mathbf{R}_{1}^{\text {ref }} \\
\mathbf{R}_{i+1}^{\text {ref }} & \mathbf{R}_{\mathbf{i}}^{\text {ref }} & \cdots & \mathbf{R}_{2}^{\text {ref }} \\
\cdots & \cdots & \cdots & \cdots \\
\mathbf{R}_{2 i-1}^{\text {ref }} & \mathbf{R}_{2 i-2}^{\text {ref }} & \cdots & \mathbf{R}_{\mathbf{i}}^{\text {ref }}
\end{array}\right]
$$

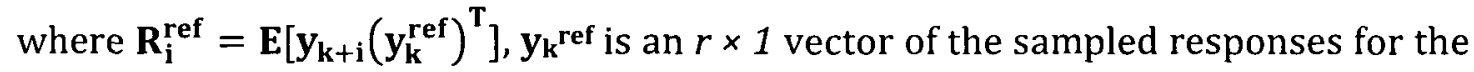
reference sensors at the time instant $k, r$ is the number of reference sensors, and $i$ is the parameter which defines the number of time lags of the correlations to be used. This parameter must satisfy the relation $r \times i>2 n$, where $2 n$ is the order of the statespace model. Based on Equation (4-10), the block Topelitz matrix can be expressed as follows:

$$
T_{1 \mid i}^{r e f}=\left[\begin{array}{c}
C \\
C A \\
\cdots \\
C A^{i-1}
\end{array}\right]\left[A^{i-1} G^{\text {ref }} \cdots A G^{\text {ref }} G^{\text {ref }}\right]=O_{i} \Gamma_{i}^{r e f}
$$

where $\mathbf{O}_{\mathbf{i}}$ is the extended observability matrix, $\Gamma_{\mathbf{i}} \mathbf{r e f}$ is the reversed extended stochastic controllability matrix, both of which are rank $2 n$. These two factors can be estimated through Singular Value Decomposition of the block Toeplitz matrix as follows:

$$
T_{1 \mid \mathrm{i}}^{\mathrm{ref}}=\mathrm{USV}^{\mathrm{T}}=\left[\begin{array}{ll}
U_{1} & U_{2}
\end{array}\right]\left[\begin{array}{cc}
S_{1} & 0 \\
0 & 0
\end{array}\right]\left[\begin{array}{l}
V_{1}^{\mathrm{T}} \\
V_{2}^{\mathrm{T}}
\end{array}\right]=U_{1} S_{1} V_{1}^{T}
$$

where $\mathbf{S}_{1}$ is a diagonal matrix containing singular values in descending order. Using Equations (4-13) and (4-14) the internally balanced realization may be obtained as follows

$$
o_{i}=U_{1} s_{1}^{1 / 2}
$$




$$
\Gamma_{i}^{r e f}=S_{1}^{1 / 2} V_{1}^{T}
$$

As the true model order of the system is unknown, solutions of increasing model orders, from 1 to $n_{\max }$, where $\mathrm{n}_{\max }$ is the maximum model order, are obtained by taking an increasing number of singular values from SVD to determine the estimates of $\mathbf{O}_{\mathbf{i}}$ and $\boldsymbol{\Gamma}_{\mathbf{i}}$ ref. For each model order, the state matrices $\mathbf{A}$ and $\mathbf{C}$ can be extracted from $\mathbf{O}_{\mathbf{i}}$ and $\boldsymbol{\Gamma}_{\mathbf{i}}$ ref. The eigenvalue decomposition of $\mathbf{A}$ yields the system's complex eigenvalues and vectors as follows:

$$
A=\boldsymbol{\Psi} \Lambda \Psi^{-1}
$$

where $\boldsymbol{\Psi}$ is the complex eigenvector matrix and $\Lambda$ is the diagonal matrix which contains the discrete-time eigenvalues $\mu_{j}$, which are directly related to the system poles $\lambda_{j}$. The poles contain the modal frequencies $\omega_{j}$ and damping ratios $\xi_{j}$ as follows:

$$
\lambda_{i}=\frac{\log _{e}\left(\mu_{i}\right)}{\Delta t}=-\xi_{i} \omega_{i}+j \sqrt{1-\xi_{i}^{2}} \omega_{i}
$$

The modal frequencies are then obtained as follows:

$$
\omega_{i}=\frac{\operatorname{imag}\left(\lambda_{i}\right)}{2 \pi}
$$

and the damping ratios are calculated as follows:

$$
\xi_{i}=\frac{-\operatorname{real}\left(\lambda_{i}\right)}{\left|\lambda_{i}\right|}
$$

The mode shapes are obtained as follows:

$$
\mathbf{V}=\mathbf{C} \Psi
$$

where $C$ can be obtained as the first $l$ rows of $\mathbf{O}_{i}$ as seen in Equation (4-13). 
Chapter 4 - Vibration Based Structural Health Monitoring

In theory the order of the system can be determined by counting the number of non-zero singular values in the matrix S. However, when analyzing real structures there is noise in the data which results in the matrix $\mathbf{S}$ having all non-zero singular values. This noise may be a result of:

- Modeling assumptions

- Measurement inaccuracies

- The use of finite data sets to compute estimates of the correlations

- Non-stationarity and non-linearity behaviour of the data

In order to overcome the uncertainty problem of an unknown system order, a stabilization diagram is constructed. For a stabilization diagram, a set of model orders are identified by including a different number of singular values and vectors from SVD in the calculation of $\mathbf{O}_{\mathbf{i}}$ and $\boldsymbol{\Gamma}_{\mathbf{i}}$. The solutions based on the different model orders are presented in the stabilization diagram, allowing for an experienced analyst to distinguish between true solutions and spurious solutions, which are a result of noise in the data. 


\subsection{Polyreference Least Squares Complex Frequency}

\section{Estimator}

While SSI has been shown to give consistent results, it remains subjective due to the influences of an analyst in the selection of poles from the stabilization diagram. In order to remove this subjectivity a technique that provides clean, welldefined stabilization diagrams is necessary. The polyreference least squares complex frequency method ( $\mathrm{p}$-LSCF) (Peeters et al. 2004a), meets this requirements. As a frequency domain technique, the p-LSCF estimator requires output spectra as primary data. It can be shown that with an input of white noise, output spectra can be modeled like frequency response functions (FRF). Heylen et al. (1997) have shown that the modal decomposition of an FRF matrix $\mathbf{H}(\omega)$ can be written as follows:

$$
H(\omega)=\sum_{k=1}^{N_{m}} \frac{v_{k} \times l_{k}^{T}}{j \omega-\lambda_{k}}+\frac{v_{k}^{*} \times l_{k}^{H}}{j \omega-\lambda_{k}^{*}}
$$

where $\mathbf{N}_{\mathrm{m}}$ is the number of modes. The notation ${ }^{*}$ denotes the complex conjugate of a matrix, ${ }^{H}$ is the complex conjugate transpose of a matrix, $\mathbf{v}_{\mathbf{k}}$ is an $l \times 1$ vector of the $\mathrm{k}^{\text {th }}$ mode shape, $\mathbf{I}_{\mathbf{k}} \mathrm{T}$ is the $1 \times m$ vector of the $\mathrm{k}^{\text {th }}$ modal participation factor and $\lambda_{\mathbf{k}}$ is the $\mathrm{k}^{\text {th }}$ system pole, which are related to the modal frequencies and damping ratios as shown in Equation (4-18). The FRF relates the input spectra $S_{u u}$ and output spectra $\mathbf{S}_{\mathrm{yy}}$ as follows: 


$$
S_{y y}(\omega)=H(\omega) S_{u u} H(\omega)^{H}
$$

As is the case of the state-space model described in Equation (4-8) a modification must be made to the deterministic formulation to account for the lack of knowledge of input forces. This is achieved by once again assuming the input is white noise. Since white noise has a constant power spectrum, it is independent of frequency (Marple, 1987). The modal decomposition can now be reformulated as follows:

$$
S_{y y}(\omega)=\sum_{k=1}^{N_{m}} \frac{v_{k} \times g_{k}}{j \omega-\lambda_{k}}+\frac{v_{k}^{*} \times g_{k}^{*}}{j \omega-\lambda_{k}^{*}}+\frac{g_{k} \times v_{k}}{-j \omega-\lambda_{k}}+\frac{g_{k}^{*} \times v_{k}^{*}}{-j \omega-\lambda_{k}^{*}}
$$

where $\mathbf{g}_{\mathrm{k}}$ are operational reference factors, which replace the modal participation factors which exist in the input-output case of experimental modal analysis.

As was the case with SSI, the correlations of the acceleration data must be estimated as per Equation (4-11). The power spectra are then defined as the Discrete Fourier Transform of the correlations:

$$
S_{y y}(\omega)=\sum_{k=-i}^{i} w_{k} R_{k} \exp (-j \omega k \Delta t)
$$

where $\mathbf{w}_{\mathbf{k}}$ is introduced as an exponential window as follows:

$$
\mathbf{w}_{\mathbf{k}}=\exp \left(-\beta \mathbf{k T}_{\mathrm{s}}\right)
$$

where $\mathbf{T}_{\mathbf{s}}$ is the sampling interval of the data and $\boldsymbol{\beta}$ is a decay factor. This window is utilized to reduce the effects of the higher variance of higher order estimates. A commonly used exponential window for p-LSCF is a $1 \%$ window, where the amplitude of the last value is reduced to $1 \%$ of its original value. Figure $4-3$ shows 
the characteristics of an exponential window. Figure 4-4 displays the effect that the exponential window has on the correlations of the data.

The use of a window adversely affects the poles of the system. More specifically artificial damping is introduced. However an exact correction can still be obtained for an exponential window (Parloo, 2003) as follows:

$$
\lambda_{r}^{\text {cor }}=\lambda_{r}^{e s t}+\beta
$$

It has been shown that in the context of modal analysis it is possible to simply calculate the half spectra of the system (Peeters et al, 2004b):

$$
S_{y y}^{+}(\omega)=\frac{w_{0} R_{0}}{2}+\sum_{k=1}^{i} w_{k} R_{k} \exp (-j \omega k \Delta t)
$$

It has been shown by Hermans et al. (1998) that the modal decomposition of the half spectra is as follows:

$$
S_{y y}^{+}(\omega)=\sum_{k=1}^{N_{m}} \frac{v_{k} \times g_{k}}{j \omega-\lambda_{k}}+\frac{v_{k}^{*} \times g_{k}^{*}}{j \omega-\lambda_{k}^{*}}
$$

The power spectra can then be modeled as a right matrix-fraction model as follows:

$$
S_{y y}^{+}(\omega)=B(\omega) \times A(\omega)^{-1}
$$

where $\mathbf{B}(\boldsymbol{\omega})$ is the complex $l \times r$ numerator matrix, and $\mathbf{A}(\boldsymbol{\omega})$ is the complex $r \times r$ denominator matrix, where $l$ is the number of outputs of the system, and $r$ is the number of selected reference sensors. Each row of the model can be formulated as:

$$
S_{y y}^{+}(\omega)_{0}=B(\omega)_{0} \times A(\omega)^{-1}
$$


where $\mathbf{B}(\boldsymbol{\omega})_{\mathrm{o}}$ and $\mathbf{A}(\boldsymbol{\omega})$ are formulated as follows:

$$
\begin{aligned}
& B(\omega)_{o}=\sum_{k=0}^{n_{\max }} \mathbf{z}_{k}(\omega) \beta_{o k} \\
& A(\omega)=\sum_{k=0}^{n_{\max }} z_{k}(\omega) \alpha_{k}
\end{aligned}
$$

where $\mathbf{z}_{\mathbf{k}}$ is the basis function:

$$
z_{k}(\omega)=\exp (j \omega k \Delta t)
$$

The coefficients can be written as follows:

$$
\begin{aligned}
& \beta_{\mathrm{o}}=\left(\begin{array}{c}
\beta_{\mathrm{o0}} \\
\beta_{\mathrm{o} 1} \\
\beta_{\mathrm{o} 2} \\
\vdots \\
\beta_{\mathrm{on}}
\end{array}\right) \\
& \alpha=\left(\begin{array}{c}
\alpha_{0} \\
\alpha_{1} \\
\alpha_{2} \\
\vdots \\
\alpha_{\mathrm{n}}
\end{array}\right) \\
& \theta=\left(\begin{array}{c}
\beta_{1} \\
\beta_{2} \\
\vdots \\
\beta_{1} \\
\alpha
\end{array}\right)
\end{aligned}
$$

where $\boldsymbol{\beta}_{\mathrm{o}}$ is an $\left(n_{\max }+1\right) \times r$ matrix which can be constructed for all outputs of the system from $0=1 \rightarrow l, \boldsymbol{\alpha}$ is an $r\left(n_{\max }+1\right) \times r$ matrix and $\boldsymbol{\theta}$ is an $(l+r)\left(n_{\max }+1\right) \times r$ matrix. 
Equation (4-30) must be solved in order to determine all the values of $\beta$ and $\boldsymbol{\alpha}$, or in this case $\boldsymbol{\theta}$. This can be achieved by formulating a non-linear least squares equation and minimizing the associated errors as follows:

$$
\begin{aligned}
& \varepsilon_{0}^{N L S}\left(\omega_{k}, \theta\right)=w_{0}\left(\omega_{k}\right)\left[S_{0}\left(\omega_{k}, \theta\right)-\widehat{S}_{0}\left(\omega_{k}\right)\right] \\
& \varepsilon_{0}^{N L S}\left(\omega_{k}, \theta\right)=w_{0}\left(\omega_{k}\right)\left[\beta_{0}\left(\omega_{k}, \theta\right) A^{-1}\left(\omega_{k}, \alpha\right)-\widehat{S}_{0}\left(\omega_{k}\right)\right]
\end{aligned}
$$

where $\mathbf{w}_{\mathbf{o}}\left(\omega_{\mathbf{k}}\right)$ is a weighting function incorporated to account for the location of the measurements defined as (Verboven, 2002):

$$
w_{0}\left(\omega_{k}\right)=\frac{\left|S_{y y}^{+}\left(\omega_{k}\right)\right|}{\operatorname{var}\left(S_{y y}^{+}\left(\omega_{k}\right)\right)}
$$

Equation (4-38) is combined for all outputs and frequencies in the following cost function:

$$
\mathrm{I}^{\mathrm{NLS}}(\theta)=\sum_{\mathbf{0}=1}^{\mathrm{l}} \sum_{\mathrm{k}=1}^{\mathrm{N}_{\mathrm{f}}} \operatorname{tr}\left[\left(\varepsilon_{\mathbf{o}}^{\mathrm{NLS}}\left(\omega_{\mathrm{k}}, \theta\right)\right)^{\mathrm{H}} \varepsilon_{\mathbf{o}}^{\mathrm{NLS}}\left(\omega_{\mathrm{k}}, \theta\right)\right]
$$

In order to approximate this non-linear problem, the non-linear least squares problem of Equation (4-38) is multiplied by the numerator matrix A, resulting in a linear least squares problem as follows:

$$
\begin{aligned}
& \varepsilon_{0}^{\mathbf{L S}}\left(\omega_{k}, \theta\right)=w_{0}\left(\omega_{k}\right)\left[B_{0}\left(\omega_{k}, \theta\right)-\hat{S}_{0}\left(\omega_{k}\right) A\left(\omega_{k}, \alpha\right)\right] \\
& \varepsilon_{0}^{\mathbf{L S}}\left(\omega_{k}, \theta\right)=w_{0}\left(\omega_{k}\right) \sum_{k=0}^{n_{\max }}\left[\mathbf{z}_{k}\left(\omega_{k}\right) \beta_{o k}-z_{k}\left(\omega_{k}\right) \hat{S}_{0}\left(\omega_{k}\right) \alpha_{k}\right]
\end{aligned}
$$

These equation errors can now be combined in a single matrix consisting of every value of $\omega$ to be taken into account: 


$$
E_{0}^{L S}(\theta)=\left[\begin{array}{c}
\varepsilon_{0}^{L S}\left(\omega_{1}, \theta\right) \\
\varepsilon_{0}^{L S}\left(\omega_{2}, \theta\right) \\
\vdots \\
\varepsilon_{0}^{L S}\left(\omega_{N_{f}}, \theta\right)
\end{array}\right]=\left[\begin{array}{ll}
X_{0} & Y_{0}
\end{array}\right]\left[\begin{array}{c}
\beta_{0} \\
\alpha
\end{array}\right]
$$

where $\mathbf{X}_{\mathbf{0}}$ and $\mathbf{Y}_{\mathbf{o}}$ are defined as follows:

$$
\begin{aligned}
& \mathbf{X}_{\mathbf{o}}=\left[\begin{array}{ccc}
\mathbf{w}_{\mathrm{o}}\left(\omega_{1}\right)\left(\mathbf{z}_{0}\left(\omega_{1}\right)\right. & \cdots & \left.\mathbf{z}_{\mathrm{n}}\left(\omega_{1}\right)\right) \\
\mathbf{w}_{\mathbf{0}}\left(\omega_{2}\right)\left(\mathbf{z}_{0}\left(\omega_{2}\right)\right. & \cdots & \left.\mathbf{z}_{\mathrm{n}}\left(\omega_{2}\right)\right) \\
\vdots & & \\
\mathbf{w}_{\mathbf{0}}\left(\boldsymbol{\omega}_{\mathrm{N}_{\mathrm{f}}}\right)\left(\mathbf{z}_{0}\left(\omega_{\mathrm{N}_{\mathrm{f}}}\right)\right. & \cdots & \left.\mathbf{z}_{\mathrm{n}}\left(\boldsymbol{\omega}_{\mathrm{N}_{\mathrm{f}}}\right)\right)
\end{array}\right]
\end{aligned}
$$

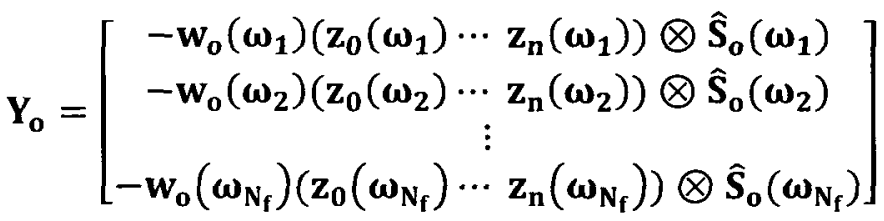

where $\otimes$ is defined as the Kronecker product, which is defined as

$$
A \otimes B=\left[\left[\begin{array}{ccc}
A_{11} B & \cdots & A_{1 n} B \\
\vdots & \ddots & \vdots \\
A_{m 1} B & \cdots & A_{m n} B
\end{array}\right]\right]
$$

where $\mathbf{A}$ is an $m \times n$ matrix and $\mathbf{B}$ is a $c \times d$ matrix, resulting in an $m c \times n d$ matrix.

The cost function of Equation (4-41) can now be formulated for the linear problem:

$$
\begin{aligned}
& l^{L S}(\theta)=\sum_{0=1}^{1} \sum_{k=1}^{N_{f}} \operatorname{tr}\left[\left(\varepsilon_{0}^{L S}\left(\omega_{k}, \theta\right)\right)^{H} \varepsilon_{0}^{L S}\left(\omega_{k}, \theta\right)\right] \\
& l^{L S}(\theta)=\sum_{0=1}^{l} \operatorname{tr}\left[\left(E_{o}^{L S}(\theta)\right)^{H} E_{o}^{L S}(\theta)\right] \\
& I^{L S}(\theta)=\sum_{0=1}^{l} \operatorname{tr}\left[\left(\beta_{0}^{T} \alpha^{T}\right)\left(\begin{array}{c}
X_{0}^{H} \\
Y_{0}^{H}
\end{array}\right)\left(X_{0} \quad Y_{0}\right)\left(\begin{array}{c}
\beta_{0} \\
\alpha
\end{array}\right)\right.
\end{aligned}
$$




$$
\mathbf{l}^{L S}(\theta)=\operatorname{tr}\left(\theta^{\mathrm{T}} J^{\mathrm{T}} J \theta\right)
$$

where $\mathbf{J}$ is the Jacobian matrix defined as:

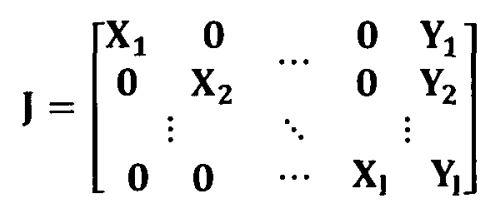

It has been shown that real part of $\mathrm{J}^{\mathrm{H} J}$ can be utilized in the cost function (Peeters et al. 2004b) to form:

$$
\mathbf{I}^{L S}(\theta)=\operatorname{tr}\left(\theta^{T} \operatorname{Re}\left(J^{H} J\right) \theta\right)
$$

where $\mathbf{R e}\left(\mathbf{J}^{\mathrm{H}}\right)$ is defined as:

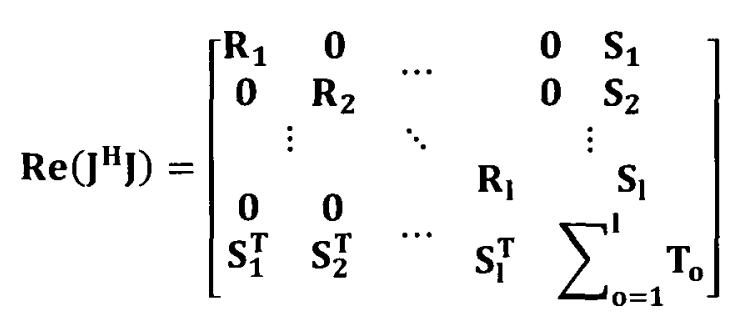

where $\mathbf{R}_{\mathbf{0}}, \boldsymbol{S}_{\mathbf{0}}$, and $\mathbf{T}_{\mathbf{0}}$ are defined as follows:

$$
\begin{aligned}
& R_{0}=\operatorname{Re}\left(X_{0}^{H} X_{0}\right) \\
& S_{0}=\operatorname{Re}\left(X_{0}^{H} Y_{0}\right) \\
& T_{0}=\operatorname{Re}\left(Y_{0}^{H} Y_{0}\right)
\end{aligned}
$$

Now the cost function of Equation (4-48) is minimized by setting the partial derivatives with respect to $\boldsymbol{\theta}$ equal to zero:

$$
\frac{\partial 1^{L S}(\theta)}{\partial \beta_{o}}=2\left(R_{0} \beta_{o}+S_{0} \alpha\right)=0
$$


Chapter 4 - Vibration Based Structural Health Monitoring

$$
\frac{\partial l^{\mathrm{LS}}(\theta)}{\partial \alpha}=2 \sum_{\mathrm{o}=1}^{\mathrm{l}}\left(\mathrm{S}_{\mathrm{o}}^{\mathrm{T}} \beta_{\mathrm{o}}+\mathrm{T}_{\mathrm{o}} \alpha\right)=0
$$

Equation (4-53) can be written synthetically using Equations (4-49), (4-35), (4-36), and (4-37).

$$
2 \operatorname{Re}\left(J^{\mathrm{H}} \mathrm{J}\right) \boldsymbol{\theta}=0
$$

As the denominator matrix $\boldsymbol{\alpha}$ contains the information for the poles and operational reference factors, $\boldsymbol{\beta}_{\mathbf{o}}$ can be eliminated from Equation (4-53), as follows:

$$
\beta_{\mathrm{o}}=-\mathbf{R}_{\mathrm{o}}^{-1} S_{\mathrm{o}} \alpha
$$

which results in:

$$
\begin{aligned}
& 2 \sum_{0=1}^{1}\left(S_{0}^{T}\left(-R_{0}^{-1}\right) S_{0}+T_{0} \alpha\right)=0 \\
& \left(2 \sum_{0=1}^{1} T_{0}-S_{0}^{T} R_{0}^{-1} S_{0}\right) \alpha=0 \\
& M \alpha=0
\end{aligned}
$$

In order to efficiently construct the necessary stabilization diagram needed to determine the stable physical poles of the system the least squares problem of Equation (4-56) is formulated for the maximum model order $\mathrm{n}_{\max }$. Following this formulation, submatrices of appropriate dimensions can be constructed for the model order $n$.

In order to solve Equation (4-56) a constraint must be placed on the system of equations to exclude the trivial solution of $\alpha=0$. The selection of this constraint 
Chapter 4 - Vibration Based Structural Health Monitoring

plays an important role in the clarity of the resulting stabilization diagrams. Figure 4-5 demonstrates the clean well-defined diagrams provided when the constraint $\alpha_{1}$ $=I_{r}$ is applied. This constraint results in negative damping values for the mathematical poles, allowing for their exclusion prior to plotting. A constraint of $\alpha_{n+1}=I_{r}$ does not provide a distinction between physical and mathematical poles with regards to damping ratios, thus all poles must be plotted, resulting in an indistinguishable diagram as shown in Figure 4-6.

Based on the constraint $\alpha_{1}=I_{r}$, the following problem can be formulated:

$$
\mathbf{A x}=\mathbf{B}
$$

where $A=M(r+1: r(n+1), r+1: r(n+1))$ and $B=-M(r+1: r(n+1), 1: r)$. Upon solving Equation (4-57) the least-squares estimate of Equation (4-56) can be written as follows:

$$
\widehat{\boldsymbol{\alpha}}_{\mathrm{LS}}=\left[\begin{array}{l}
\mathbf{I}_{\mathbf{r}} \\
\mathbf{X}
\end{array}\right]
$$

The discrete-time poles are then found as the eigenvalues of the following companion matrix:

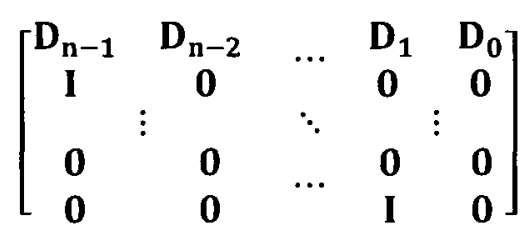

where $\mathbf{D}_{\mathbf{j}}=-\widehat{\boldsymbol{\alpha}}_{\mathbf{n}}^{-1} \boldsymbol{\alpha}_{\mathbf{j}}$.

The last $m$ rows of the eigenvector matrix correspond to the operational reference factors. 


\subsubsection{Validation}

In this section validation of the implementation of the polyreference least squares complex frequency estimator algorithm is presented. Nineteen separate sine waves of varying frequency and damping ratios have been used as input to the system. The modal frequencies and damping ratios were estimated using both the stochastic subspace identification method and the polyreference least squares complex frequency estimator. Table 4-1 shows excellent results are obtained by the p-LSCF estimator, with a maximum error of $0.0217 \%$ for the modal frequency, and $1.0500 \%$ for the damping ratio. As can be seen from Table 4-2 to Table $4-6$, the results obtained by SSI varied greatly depending upon the number of reference channels selected. Using a typical number of reference channels, three, only six poles are distinguishable, with a maximum error of $9.6361 \%$ for modal frequency and $341.8333 \%$ for damping ratio. An increase in the number of reference channels increases both the number of poles and the accuracy of the poles. However, even when using a large number of reference channels, fifteen, not all poles can be determined by the system. Using the p-LSCF estimator, all poles can be determined accurately with only three reference channels selected. These results by the p-LSCF estimator clearly are more robust and have higher accuracy than those by the stochastic subspace identification technique in identification of all physical poles from measured response data. The need of a large number of reference channels, 
such as fifteen in the SSI technique, requires an undesirable amount of processing memory and computing effort.

In the past, it was thought that a time-domain technique is required when closely spaced poles are potentially present in the data. This is due to the fact that frequency-domain techniques often use operational deflection shapes when distinguishing between poles. These operational deflection shapes can be the superposition of several mode shapes when there are closely spaced poles (Peeters et al. 2007). The p-LSCF estimator does not fall victim to such deficiencies however, as it uses operational reference factors, not operational deflection shapes, when distinguishing between poles. This allows for the accurate determination of closely spaced poles, as shown in Table 4-7 and Figure 4-7. As Figure 4-8 shows, SSI identifies two physical poles around $2 \mathrm{~Hz}$. However the results are not as accurate as those from the p-LSCF estimator, an $8.8109 \%$ error for the modal frequency and an $83.52 \%$ error for the damping ratio.

The use of the exponential window when determining the half power spectra induces artificial damping onto the system. This bias on the damping can be corrected by modifying the discrete-time poles of the system, as demonstrated by Equation (4-27). As the correction is performed by addition of a real number, the modal frequencies are not affected. The results of this correction are evident in Table $4-8$, which shows a maximum error of only $1.0500 \%$ when the correction is used, as compared to $495.3100 \%$ when no correction is used. 
Chapter 4 - Vibration Based Structural Health Monitoring

In an attempt to create a more realistic set of validation data, white Gaussian noise is added to the inputted sine wave data, as shown in Figure 4-9. Table 4-9 presents the results obtained by the p-LSCF estimator, which show an expected increase in the error of the determined frequencies. However the maximum error is only $0.16 \%$. It should be noted that the error associated with the determined damping ratios has grown to a maximum of $11.15 \%$.

\subsection{Automatic Pole Selection}

The need for advancement in vibration based structural health monitoring is evident due to the limitations of the current common practice of condition assessment of bridges, manual visual inspection. Since manual visual inspection of a bridge is typically carried out every two years it is not as effective as remote continuous monitoring by vibration based structural health monitoring techniques which can provide up to date condition assessment information of the monitored bridge and thus can allow for more rapid remedial actions. In addition, the accuracy and reliability of visual inspection results are subjective to the experiences and judgment of the inspectors. In order to improve the reliability and services provided by today's bridge infrastructure, advancement in current practice of bridge condition assessment is needed. The development of vibration based structural health monitoring techniques has garnered much attention in recent 
years. While the current system identification techniques utilized for the determination of the modal properties of a structure from measured vibration response data reduce the subjectivity and improve the consistency of the bridge condition information compared to manual visual inspection, the current system identification techniques still require the input of an analyst. In the monitoring software platform SPPLASH, the stochastic subspace identification algorithm requires the analyst to select the physical poles from a stabilization diagram. This requirement of direct intervention and input by an analyst in the data analysis process seriously limits the capability of the monitoring system to operate in a continuous automatic manner which is critical in an intelligent bridge monitoring early warning system. To improve the robustness, reliability, and accuracy of vibration based structural health monitoring techniques, and to advance the development of an intelligent bridge monitoring early warning system, there is the need to formulate an automatic procedure for the determination of the modal vibration properties of a structure through the analysis of measured vibration response data. Examples of previous automatic procedures are found in the references Lanslots et al. (2004) and Van der Auweraer and Peeters (2004).

The most crucial aspect in automating the selection of physical poles from a stabilization diagram is clarity of the diagram. Previous sections of Chapter 4 present the advantage of the p-LSCF estimator as compared to the stochastic subspace identification technique in the accuracy and reliability of the system identification results and robustness of the analysis process. The p-LSCF estimator 
procedure has the capability of generating well defined stabilization diagrams for more accurate and automatic pole selection.

In the stochastic subspace identification procedure, an experienced analyst examines the stabilization diagram derived from processing and analysis of the vibration response monitoring data to identify all the consistently stable physical poles in the data with increasing model orders. The lowest model order containing all the stable poles is then selected for the state-space model of the monitored structure. In this present study, an automatic pole selection algorithm is formulated and implemented in the monitoring software platform SPPLASH.

The initial step in the automated process is the determination of test frequencies against which all other frequencies of each model order are to be tested. With the improved clarity in the stabilization diagrams generated by the p-LSCF algorithm, as shown in Figure 4-10, the determination of these test frequencies is relatively simple. Four model orders are selected, from which each frequency is compared with each frequency in the remaining three model orders. Should a frequency be found within a specified small tolerance in all four model orders, then it is selected as an appropriate test frequency. The use of four model orders ensures that the test frequency is not a spurious or random pole due to noise or other uncertainty in the data.

Following the determination of test frequencies, the first test frequency $T_{1}$, from the set of all test frequencies $T_{n}$ is selected for validation. This frequency, $T_{1}$, is compared with each frequency of every model order from 85 to $n_{\max }=150$. The 
value of 85 was determined by the author following the analysis of a series of stabilization diagrams based on the p-LSCF estimator which displayed a need of at least this model order to ensure all physical poles would begin to stabilize. If the percentage difference of the test frequency and a pole at the selected model order falls within a specified tolerance, and the pole is determined to be stable in terms of frequency, damping, and operational reference factor, then the pole is stored as a possible physical pole. This process is then repeated for all model orders up to $n_{\max }$. An average of all stored possible poles is then calculated. The model orders of the selected stable poles are then examined to determine if a predetermined number of stable poles, $\mathrm{N}_{\mathrm{p}}$, occur for consecutive model orders. If this is the case the pole is selected as a true physical pole, otherwise it is discarded. Following the determination of a true physical pole, the stable pole which is closest to the average value of all poles is determined and selected as the best fit for the single pole value of that frequency. The above procedure is repeated for each test frequency determined in the initial step. Following a repetition of this process, a group of physical poles and the corresponding model orders are determined. The algorithm then determines the lowest model order of those selected at which all physical poles are stable. Figure 4-11 outlines the procedure performed by the automated pole selection algorithm. 


\subsubsection{Validation}

The validity of the automated pole selection procedure is verified through analysis of sine wave data. The same input used in the validation of the p-LSCF estimator presented section 4.4 .1 is used here for consistency. Initially the sine wave data is inputted as exact values, with no noise present. Table 4-10 shows the excellent results provided by the procedure. It can be seen that all poles are correctly selected, including the closely spaced poles, while no spurious poles are selected, and each physical pole is only selected once. In order to evaluate the accuracy and sensitivity of the automatic pole selection procedure, more realistic data are used by adding white Gaussian noise the sine wave data. Table 4-11 shows the excellent results provided by the automated pole selection procedure in the presence of white noise, where once again all physical poles are correctly selected, and no incorrect selections are made.

To further verify the robust nature of the automated procedure a comparison is made using measured vibration response data of the Confederation Bridge. For the comparison, three groups of users are considered:

1) Novice

2) Experienced

3) Automated

Ten datasets are analyzed to ensure a wide variety of data are tested. The experienced user is used as the benchmark against which the other two types of 
user are measured. The results are shown in Table 4-12 and Figure 4-12. The novice user selected 103 stable physical poles, while the automated procedure and experienced user selected the same 106 stable physical poles. The discrepancy between the experience user and the novice is small, demonstrating the clarity of the p-LSCF estimator based stabilization diagrams. A second comparison is performed based solely on the experienced user and the automated selection procedure. For this comparison twenty-two datasets are analyzed. The experienced user selected a total of 232 stable physical poles, while the automated procedure selected 234 stable physical poles. The two extra poles selected by the automated procedure are extra, incorrect poles. These results demonstrate the ability of the automated pole selection procedure to accurately and reliably determine all physical poles from measured vibration response data. 
Table 4-1 : $\mathrm{p}$-LSCF validation results using 3 reference sensors

\begin{tabular}{|c|c|c|c|c|c|}
\hline \multicolumn{3}{|c|}{ Frequency } & \multicolumn{3}{c|}{ Damping Ratio } \\
\hline \hline $\begin{array}{c}\text { Input } \\
\sin (\omega \mathrm{t})\end{array}$ & $\begin{array}{c}\text { Extracted } \\
\omega\end{array}$ & $\%$ Error & $\begin{array}{c}\text { Input } \\
\mathrm{e}^{-\xi \omega t}\end{array}$ & $\begin{array}{c}\text { Extracted } \\
\xi\end{array}$ & \% Error \\
\hline 0.7 & 0.69998 & 0.0029 & 5 & 4.9843 & 0.3140 \\
\hline 1.4 & 1.4001 & 0.0071 & 4 & 3.9894 & 0.2650 \\
\hline 2 & 2.0001 & 0.0050 & 2.5 & 2.4764 & 0.9440 \\
\hline 2.3 & 2.3005 & 0.0217 & 7 & 6.9265 & 1.0500 \\
\hline 3 & 3.0001 & 0.0033 & 4 & 3.9945 & 0.1375 \\
\hline 3.6 & 3.6 & 0 & 3 & 2.9987 & 0.0433 \\
\hline 4.5 & 4.5 & 0 & 7 & 6.9829 & 0.2443 \\
\hline 5.6 & 5.6 & 0 & 8 & 7.9745 & 0.3188 \\
\hline 6 & 6 & 0 & 2 & 1.9999 & 0.0050 \\
\hline 6.9 & 6.9 & 0 & 6 & 5.9891 & 0.1817 \\
\hline 7.8 & 7.8 & 0 & 4.4 & 4.3954 & 0.1045 \\
\hline 8 & 8 & 0 & 4 & 3.9959 & 0.1025 \\
\hline 9 & 9 & 0 & 3 & 2.9986 & 0.0467 \\
\hline 10.34 & 10.34 & 0 & 7.5 & 7.4784 & 0.2880 \\
\hline 11.3 & 11.3 & 0 & 5 & 4.9936 & 0.1280 \\
\hline 12.6 & 12.6 & 0 & 4 & 3.9967 & 0.0825 \\
\hline 13.85 & 13.85 & 0 & 1.5 & 1.4998 & 0.0133 \\
\hline 14 & 14 & 0 & 3.2 & 3.1981 & 0.0594 \\
\hline 18.1 & 18.1 & 0 & 4 & 3.9968 & 0.0800 \\
\hline
\end{tabular}


Table 4-2: SSI validation results using 3 references sensors

\begin{tabular}{|c|c|c|c|c|c|}
\hline \multicolumn{3}{|c|}{ Frequency } & \multicolumn{3}{c|}{ Damping Ratio } \\
\hline \hline $\begin{array}{c}\text { Input } \\
\sin (\omega \mathrm{t})\end{array}$ & $\begin{array}{c}\text { Extracted } \\
\omega\end{array}$ & $\%$ Error & $\begin{array}{c}\text { Input } \\
\mathrm{e}^{-\xi \omega t}\end{array}$ & $\begin{array}{c}\text { Extracted } \\
\xi\end{array}$ & $\%$ Error \\
\hline 0.7 & 0.70001 & 0.0014 & 5 & 4.9964 & 0.0720 \\
\hline 1.4 & 1.4003 & 0.0214 & 4 & 4.008 & 0.2000 \\
\hline 2 & 2.0003 & 0.0150 & 2.5 & 2.5045 & 0.1800 \\
\hline 2.3 & 2.0856 & 9.3217 & 7 & 10.5991 & 51.4157 \\
\hline 3 & & & 4 & & \\
\hline 3.6 & 3.9469 & 9.6361 & 3 & 13.255 & 341.8333 \\
\hline 4.5 & & & 7 & & \\
\hline 5.6 & 5.5318 & 1.2179 & 8 & 12.9691 & 62.1138 \\
\hline 6 & & & 2 & & \\
\hline 6.9 & & & 6 & & \\
\hline 7.8 & & & 4.4 & & \\
\hline 8 & & & 4 & & \\
\hline 9 & & & 3 & & \\
\hline 10.34 & & & 7.5 & & \\
\hline 11.3 & & & 5 & & \\
\hline 12.6 & & & 4 & & \\
\hline 13.85 & & & 1.5 & & \\
\hline 14 & & & 3.2 & & \\
\hline 18.1 & & & 4 & & \\
\hline
\end{tabular}


Table 4-3: SSI validation results using 6 reference sensors

\begin{tabular}{|c|c|c|c|c|c|}
\hline \multicolumn{3}{|c|}{ Frequency } & \multicolumn{3}{|c|}{ Damping Ratio } \\
\hline $\begin{array}{l}\text { Input } \\
\sin (\omega t)\end{array}$ & $\begin{array}{c}\text { Extracted } \\
\omega\end{array}$ & $\%$ Error & $\begin{array}{l}\text { Input } \\
e^{-\xi \omega t} \\
\end{array}$ & $\begin{array}{c}\text { Extracted } \\
\xi\end{array}$ & $\%$ Error \\
\hline 0.7 & 0.7 & 0.0000 & 5 & 4.9941 & 0.1180 \\
\hline 1.4 & 1.4 & 0.0000 & 4 & 3.998 & 0.0500 \\
\hline 2 & 2 & 0.0000 & 2.5 & 2.4999 & 0.0040 \\
\hline 2.3 & 2.5514 & 10.9304 & 7 & 11.6085 & 65.8357 \\
\hline 3 & 3.0185 & 0.6167 & 4 & 4.567 & 14.1750 \\
\hline 3.6 & 3.6093 & 0.2583 & 3 & 3.114 & 3.8000 \\
\hline 4.5 & & & 7 & & \\
\hline 5.6 & & & 8 & & \\
\hline 6 & & & 2 & & \\
\hline 6.9 & & & 6 & & \\
\hline 7.8 & & & 4.4 & & \\
\hline 8 & & & 4 & & \\
\hline 9 & & & 3 & & \\
\hline 10.34 & & & 7.5 & & \\
\hline 11.3 & & & 5 & & \\
\hline 12.6 & & & 4 & & \\
\hline 13.85 & & & 1.5 & & \\
\hline 14 & & & 3.2 & & \\
\hline 18.1 & & & 4 & & \\
\hline
\end{tabular}


Chapter 4 - Vibration Based Structural Health Monitoring

Table 4-4 : SSI validation results using 9 references sensors

\begin{tabular}{|c|c|c|c|c|c|}
\hline \multicolumn{3}{|c|}{ Frequency } & \multicolumn{3}{c|}{ Damping Ratio } \\
\hline \hline $\begin{array}{c}\text { Input } \\
\sin (\omega \mathrm{t})\end{array}$ & $\begin{array}{c}\text { Extracted } \\
\omega\end{array}$ & $\%$ Error & $\begin{array}{c}\text { Input } \\
\mathrm{e}^{-\xi \omega \mathrm{t}}\end{array}$ & $\begin{array}{c}\text { Extracted } \\
\xi\end{array}$ & $\%$ Error \\
\hline 0.7 & 0.7 & 0 & 5 & 4.9938 & 0.1240 \\
\hline 1.4 & 1.4 & 0 & 4 & 3.9968 & 0.0800 \\
\hline 2 & 2 & 0 & 2.5 & 2.4992 & 0.0320 \\
\hline 2.3 & 2.3 & 0 & 7 & 6.9833 & 0.2386 \\
\hline 3 & 3 & 0 & 4 & 3.9969 & 0.0775 \\
\hline 3.6 & 3.6 & 0 & 3 & 2.9987 & 0.0433 \\
\hline 4.5 & 4.5416 & 0.9244 & 7 & 7.594 & 8.4857 \\
\hline 5.6 & & & 8 & & \\
\hline 6 & 6 & 0 & 2 & 1.9996 & 0.0200 \\
\hline 6.9 & & & 6 & & \\
\hline 7.8 & & & 4.4 & & \\
\hline 8 & & & 4 & & \\
\hline 9 & 9.4648 & 5.1644 & 3 & 5.1499 & 71.6633 \\
\hline 10.34 & & & 7.5 & & \\
\hline 11.3 & 11.1252 & 1.5469 & 5 & 2.869 & 42.6200 \\
\hline 12.6 & & & 4 & & \\
\hline 13.85 & & & 1.5 & & \\
\hline 14 & & & 3.2 & & \\
\hline 18.1 & & & 4 & & \\
\hline
\end{tabular}


Chapter 4 - Vibration Based Structural Health Monitoring

Table 4-5 : SSI validation results using 12 reference sensors

\begin{tabular}{|c|c|c|c|c|c|}
\hline \multicolumn{3}{|c|}{ Frequency } & \multicolumn{3}{c|}{ Damping Ratio } \\
\hline \hline $\begin{array}{c}\text { Input } \\
\sin (\omega \mathrm{t})\end{array}$ & $\begin{array}{c}\text { Extracted } \\
\omega\end{array}$ & $\%$ Error & $\begin{array}{c}\text { Input } \\
e^{-\xi \omega t}\end{array}$ & $\begin{array}{c}\text { Extracted } \\
\xi\end{array}$ & $\%$ Error \\
\hline 0.7 & 0.7 & 0 & 5 & 4.9938 & 0.1240 \\
\hline 1.4 & 1.4 & 0 & 4 & 3.9968 & 0.0800 \\
\hline 2 & 2 & 0 & 2.5 & 2.4992 & 0.0320 \\
\hline 2.3 & 2.3 & 0 & 7 & 6.9828 & 0.2457 \\
\hline 3 & 3 & 0 & 4 & 3.9968 & 0.0800 \\
\hline 3.6 & 3.6 & 0 & 3 & 2.9987 & 0.0433 \\
\hline 4.5 & 4.5003 & 0.00667 & 7 & 6.9881 & 0.1700 \\
\hline 5.6 & & & 8 & & \\
\hline 6 & 6 & 0 & 2 & 1.9996 & 0.0200 \\
\hline 6.9 & 7.1671 & 3.8710 & 6 & 6.0658 & 1.0967 \\
\hline 7.8 & & & 4.4 & & \\
\hline 8 & 7.9477 & 0.6537 & 4 & 4.1377 & 3.4425 \\
\hline 9 & 8.9843 & 0.1744 & 3 & 3.0651 & 2.1700 \\
\hline 10.34 & & & 7.5 & & \\
\hline 11.3 & & & 5 & & \\
\hline 12.6 & & & 4 & & \\
\hline 13.85 & 13.8402 & 0.0708 & 1.5 & 1.5747 & 4.9800 \\
\hline 14 & & & 3.2 & & \\
\hline 18.1 & & & 4 & & \\
\hline
\end{tabular}


Table 4-6 : SSI validation results using 15 reference sensors

\begin{tabular}{|c|c|c|c|c|c|}
\hline \multicolumn{3}{|c|}{ Frequency } & \multicolumn{3}{c|}{ Damping Ratio } \\
\hline \hline $\begin{array}{c}\text { Input } \\
\sin (\omega \mathrm{t})\end{array}$ & $\begin{array}{c}\text { Extracted } \\
\omega\end{array}$ & $\%$ Error & $\begin{array}{c}\text { Input } \\
\mathrm{e}^{-\xi \omega t}\end{array}$ & $\begin{array}{c}\text { Extracted } \\
\xi\end{array}$ & $\%$ Error \\
\hline 0.7 & 0.7 & 0 & 5 & 4.9938 & 0.1240 \\
\hline 1.4 & 1.4 & 0 & 4 & 3.9968 & 0.0800 \\
\hline 2 & 2 & 0 & 2.5 & 2.4992 & 0.0320 \\
\hline 2.3 & 2.3 & 0 & 7 & 6.9829 & 0.2443 \\
\hline 3 & 3 & 0 & 4 & 3.9968 & 0.0800 \\
\hline 3.6 & 3.6 & 0 & 3 & 2.9987 & 0.0433 \\
\hline 4.5 & 4.5 & 0 & 7 & 6.983 & 0.2429 \\
\hline 5.6 & 5.6006 & 0.01071 & 8 & & \\
\hline 6 & 6 & 0 & 2 & 1.9996 & 0.0200 \\
\hline 6.9 & 6.9001 & 0.00145 & 6 & 5.9906 & 0.1567 \\
\hline 7.8 & 7.8 & 0 & 4.4 & 4.3959 & 0.0932 \\
\hline 8 & 8 & 0 & 4 & 3.9969 & 0.0775 \\
\hline 9 & 9 & 0 & 3 & 2.9987 & 0.0433 \\
\hline 10.34 & & & 7.5 & & \\
\hline 11.3 & & & 5 & & \\
\hline 12.6 & 12.1699 & 3.41349 & 4 & 4.7756 & 19.3900 \\
\hline 13.85 & 13.85 & 0 & 1.5 & 1.4998 & 0.0133 \\
\hline 14 & & & 3.2 & & \\
\hline 18.1 & & & 4 & & \\
\hline
\end{tabular}


$N$

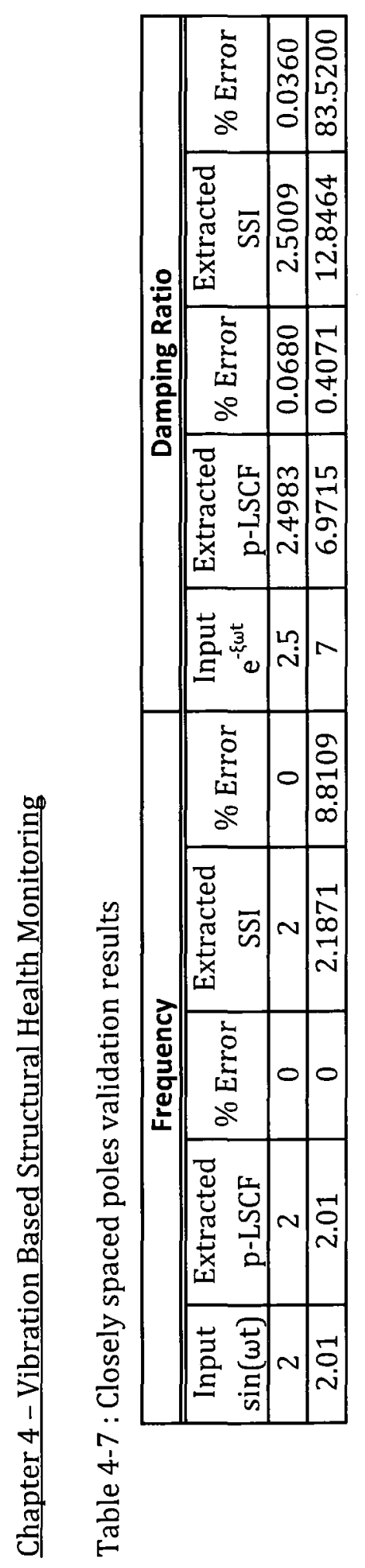


Table 4-8 : Effect of exponential window correction

\begin{tabular}{|c|c|c|c|c|}
\hline Input & \multicolumn{2}{|c|}{ No Beta } & \multicolumn{2}{c|}{ Beta } \\
\hline \hline $\mathrm{e}^{-\xi \omega t}$ & $\begin{array}{c}\text { Extracted } \\
\xi\end{array}$ & $\%$ Error & $\begin{array}{c}\text { Extracted } \\
\xi\end{array}$ & \% Error \\
\hline 5 & 29.7655 & 495.3100 & 4.9843 & 0.3140 \\
\hline 4 & 16.8409 & 321.0225 & 3.9894 & 0.2650 \\
\hline 2.5 & 11.5501 & 362.0040 & 2.4764 & 0.9440 \\
\hline 7 & 15.8180 & 125.9714 & 6.9265 & 1.0500 \\
\hline 4 & 10.0551 & 151.3775 & 3.9945 & 0.1375 \\
\hline 3 & 8.0633 & 168.7767 & 2.9987 & 0.0433 \\
\hline 7 & 11.0047 & 57.2100 & 6.9829 & 0.2443 \\
\hline 8 & 11.2011 & 40.0138 & 7.9745 & 0.3188 \\
\hline 2 & 5.0475 & 152.3750 & 1.9999 & 0.0050 \\
\hline 6 & 8.6233 & 43.7217 & 5.9891 & 0.1817 \\
\hline 4.4 & 6.7336 & 53.0364 & 4.3954 & 0.1045 \\
\hline 4 & 6.2779 & 56.9475 & 3.9959 & 0.1025 \\
\hline 3 & 5.0296 & 67.6533 & 2.9986 & 0.0467 \\
\hline 7.5 & 9.2319 & 23.0920 & 7.4784 & 0.2880 \\
\hline 5 & 6.6069 & 32.1380 & 4.9936 & 0.1280 \\
\hline 4 & 5.4461 & 36.1525 & 3.9967 & 0.0825 \\
\hline 1.5 & 2.8218 & 88.1200 & 1.4998 & 0.0133 \\
\hline 3.2 & 4.504 & 40.7500 & 3.1981 & 0.0594 \\
\hline 4 & 5.006 & 25.1500 & 3.9968 & 0.0800 \\
\hline
\end{tabular}


Table 4-9 : p-LSCF estimator results with added white Gaussian noise

\begin{tabular}{|c|c|c|c|c|c|}
\hline \multicolumn{3}{|c|}{ Frequency } & \multicolumn{3}{c|}{ Damping Ratio } \\
\hline \hline $\begin{array}{c}\text { Input } \\
\sin (\omega \mathrm{t})\end{array}$ & $\begin{array}{c}\text { Extracted } \\
\omega\end{array}$ & $\%$ Error & $\begin{array}{c}\text { Input } \\
\mathrm{e}^{-\xi \omega t}\end{array}$ & $\begin{array}{c}\text { Extracted } \\
\omega\end{array}$ & $\%$ Error \\
\hline 0.7 & 0.69965 & 0.05 & 5 & 4.8104 & 3.792 \\
\hline 1.4 & 1.3993 & 0.05 & 4 & 3.9604 & 0.99 \\
\hline 2 & 1.9955 & 0.225 & 2.5 & 2.4548 & 1.808 \\
\hline 2.01 & 2.0165 & 0.3234 & 7 & 6.4279 & 8.1729 \\
\hline 3 & 3.0003 & 0.01 & 4 & 3.9879 & 0.3025 \\
\hline 3.6 & 3.6004 & 0.01111 & 3 & 2.9825 & 0.5833 \\
\hline 4.5 & 4.5014 & 0.03111 & 7 & 6.7887 & 3.0186 \\
\hline 5.6 & 5.6043 & 0.07679 & 8 & 7.4949 & 6.3138 \\
\hline 6 & 5.9974 & 0.04333 & 2 & 2.008 & 0.4 \\
\hline 6.9 & 6.8956 & 0.06377 & 6 & 5.8489 & 2.5183 \\
\hline 7.8 & 7.7877 & 0.15769 & 4.4 & 3.9393 & 10.4705 \\
\hline 8 & 7.998 & 0.025 & 4 & 3.7718 & 5.705 \\
\hline 9 & 9.0034 & 0.03778 & 3 & 2.9701 & 0.9967 \\
\hline 10.34 & 10.341 & 0.00967 & 7.5 & 6.6641 & 11.1453 \\
\hline 11.3 & 11.315 & 0.13274 & 5 & 4.8233 & 3.534 \\
\hline 12.6 & 12.603 & 0.02381 & 4 & 3.9323 & 1.6925 \\
\hline 13.85 & 13.853 & 0.02166 & 1.5 & 1.4839 & 1.0733 \\
\hline 14 & 14.003 & 0.02143 & 3.2 & 3.0414 & 4.9563 \\
\hline 18.1 & 18.103 & 0.01657 & 4 & 3.8894 & 2.765 \\
\hline
\end{tabular}


Table 4-10: Selected poles based on automatic pole selection procedure

\begin{tabular}{|c|c|c|c|c|c|}
\hline \multicolumn{3}{|c|}{ Frequency } & \multicolumn{3}{c|}{ Damping Ratio } \\
\hline \hline $\begin{array}{c}\text { Input } \\
\sin (\omega \mathrm{t})\end{array}$ & $\begin{array}{c}\text { Extracted } \\
\omega\end{array}$ & \% Error & $\begin{array}{c}\text { Input } \\
\mathrm{e}^{-\xi \omega t}\end{array}$ & $\begin{array}{c}\text { Extracted } \\
\xi\end{array}$ & $\%$ Error \\
\hline 0.7 & 0.70001 & 0.0014 & 5 & 4.9905 & 0.1900 \\
\hline 1.4 & 1.4 & 0.0000 & 4 & 3.9935 & 0.1625 \\
\hline 2 & 2.0001 & 0.0050 & 2.5 & 2.4938 & 0.2480 \\
\hline 2.01 & 2.0101 & 0.0050 & 7 & 6.9748 & 0.3600 \\
\hline 3 & 3 & 0.0000 & 4 & 3.9971 & 0.0725 \\
\hline 3.6 & 3.6 & 0 & 3 & 2.9989 & 0.0367 \\
\hline 4.5 & 4.5 & 0 & 7 & 6.9829 & 0.2443 \\
\hline 5.6 & 5.6 & 0 & 8 & 7.9746 & 0.3175 \\
\hline 6 & 6 & 0 & 2 & 1.9997 & 0.0150 \\
\hline 6.9 & 6.9 & 0 & 6 & 5.9893 & 0.1783 \\
\hline 7.8 & 7.8 & 0 & 4.4 & 4.3958 & 0.0955 \\
\hline 8 & 8 & 0 & 4 & 3.9968 & 0.0800 \\
\hline 9 & 9 & 0 & 3 & 2.9987 & 0.0433 \\
\hline 10.34 & 10.34 & 0 & 7.5 & 7.479 & 0.2800 \\
\hline 11.3 & 11.3 & 0 & 5 & 4.9938 & 0.1240 \\
\hline 12.6 & 12.6 & 0 & 4 & 3.9968 & 0.0800 \\
\hline 13.85 & 13.85 & 0 & 1.5 & 1.4998 & 0.0133 \\
\hline 14 & 14 & 0 & 3.2 & 3.1984 & 0.0500 \\
\hline 18.1 & 18.1 & 0 & 4 & 3.9968 & 0.0800 \\
\hline
\end{tabular}


Table 4-11 : Selected poles from sine wave data including white Gaussian noise based on automatic pole selection procedure

\begin{tabular}{|c|c|c|c|c|c|}
\hline \multicolumn{3}{|c|}{ Frequency } & \multicolumn{3}{c|}{ Damping Ratio } \\
\hline \hline $\begin{array}{c}\text { Input } \\
\sin (\omega t)\end{array}$ & $\begin{array}{c}\text { Extracted } \\
\omega\end{array}$ & $\%$ Error & $\begin{array}{c}\text { Input } \\
\mathrm{e}^{-\xi \omega t}\end{array}$ & $\begin{array}{c}\text { Extracted } \\
\xi\end{array}$ & $\%$ Error \\
\hline 0.7 & 0.69965 & 0.0500 & 5 & 4.8104 & 3.7920 \\
\hline 1.4 & 1.3993 & 0.0500 & 4 & 3.9604 & 0.9900 \\
\hline 2 & 1.9955 & 0.2250 & 2.5 & 2.4548 & 1.8080 \\
\hline 2.01 & 2.0165 & 0.3234 & 7 & 6.4279 & 8.1729 \\
\hline 3 & 3.0003 & 0.0100 & 4 & 3.9879 & 0.3025 \\
\hline 3.6 & 3.6004 & 0.01111 & 3 & 2.9825 & 0.5833 \\
\hline 4.5 & 4.5014 & 0.03111 & 7 & 6.7887 & 3.0186 \\
\hline 5.6 & 5.6043 & 0.07679 & 8 & 7.4949 & 6.3138 \\
\hline 6 & 5.9974 & 0.04333 & 2 & 2.008 & 0.4000 \\
\hline 6.9 & 6.8956 & 0.06377 & 6 & 5.8489 & 2.5183 \\
\hline 7.8 & 7.7877 & 0.15769 & 4.4 & 3.9393 & 10.4705 \\
\hline 8 & 7.998 & 0.025 & 4 & 3.7718 & 5.7050 \\
\hline 9 & 9.0034 & 0.03778 & 3 & 2.9701 & 0.9967 \\
\hline 10.34 & 10.341 & 0.00967 & 7.5 & 6.6641 & 11.1453 \\
\hline 11.3 & 11.315 & 0.13274 & 5 & 4.8233 & 3.5340 \\
\hline 12.6 & 12.603 & 0.02381 & 4 & 3.9323 & 1.6925 \\
\hline 13.85 & 13.853 & 0.02166 & 1.5 & 1.4839 & 1.0733 \\
\hline 14 & 14.003 & 0.02143 & 3.2 & 3.0414 & 4.9563 \\
\hline 18.1 & 18.103 & 0.01657 & 4 & 3.8894 & 2.7650 \\
\hline
\end{tabular}


Chapter 4 - Vibration Based Structural Health Monitoring

Table 4-12: Comparison of user types for selection of poles

\begin{tabular}{|c|c|c|c|c|}
\hline \multirow{2}{*}{ User } & \multicolumn{2}{|c|}{ Comparison \#1 } & \multicolumn{2}{c|}{ Comparison \#2 } \\
\cline { 2 - 5 } & Selected Poles & Percentage Error & Selected Poles & Percentage Error \\
\hline \hline 1 & 103 & 2.83 & - & - \\
2 & 106 & 0.00 & 232 & 0 \\
3 & 106 & 0.00 & 234 & 0.86 \\
\hline
\end{tabular}




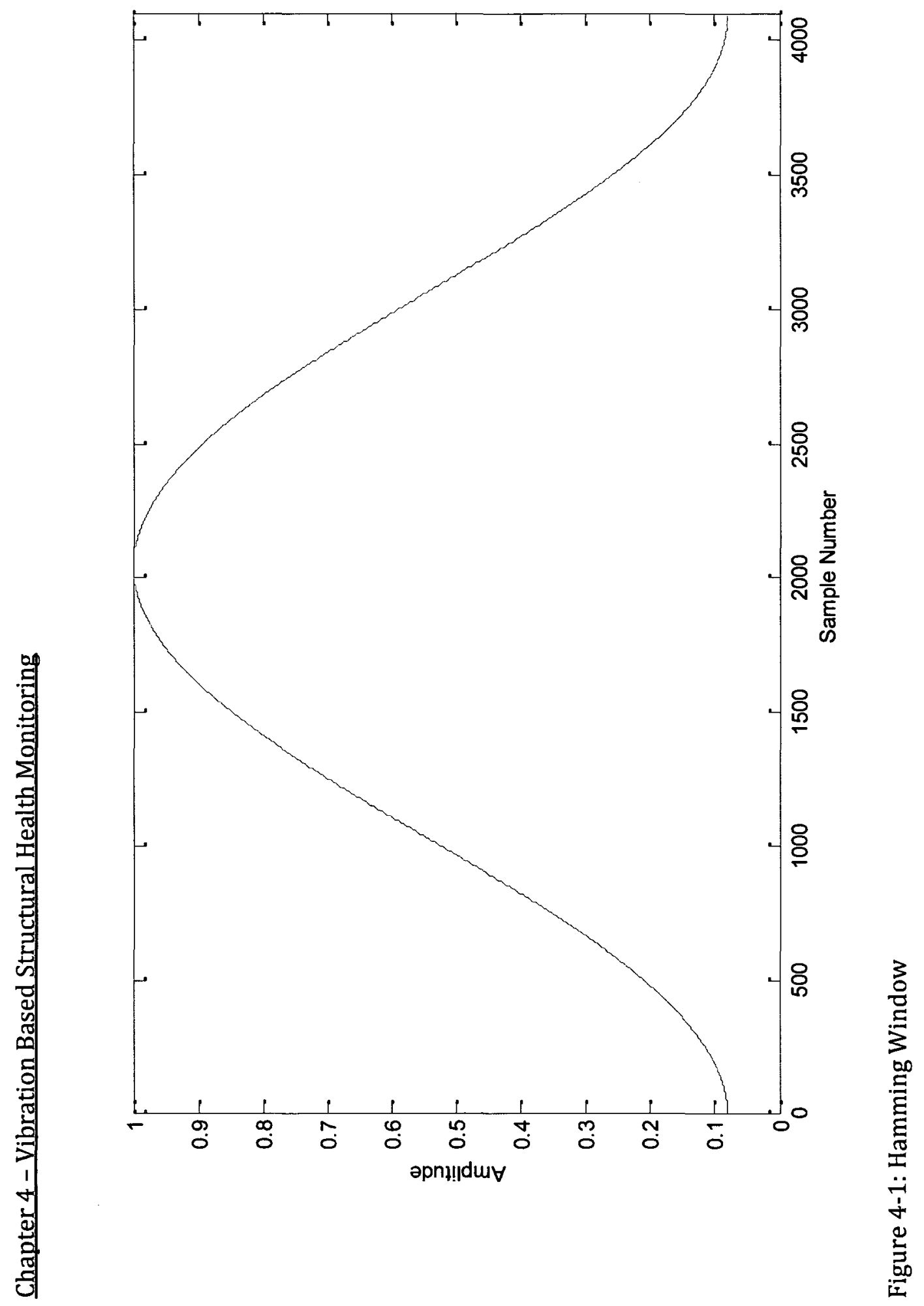




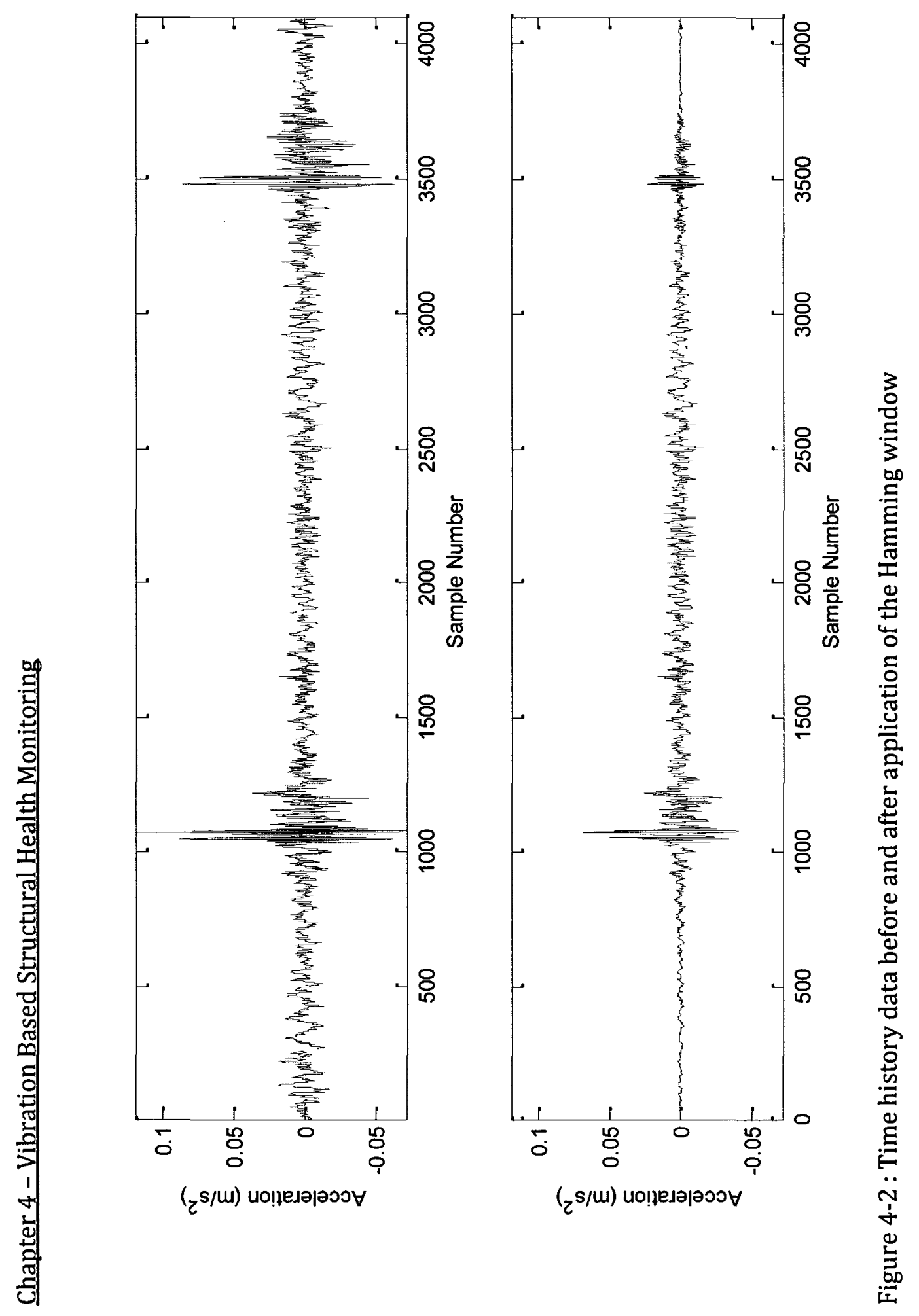




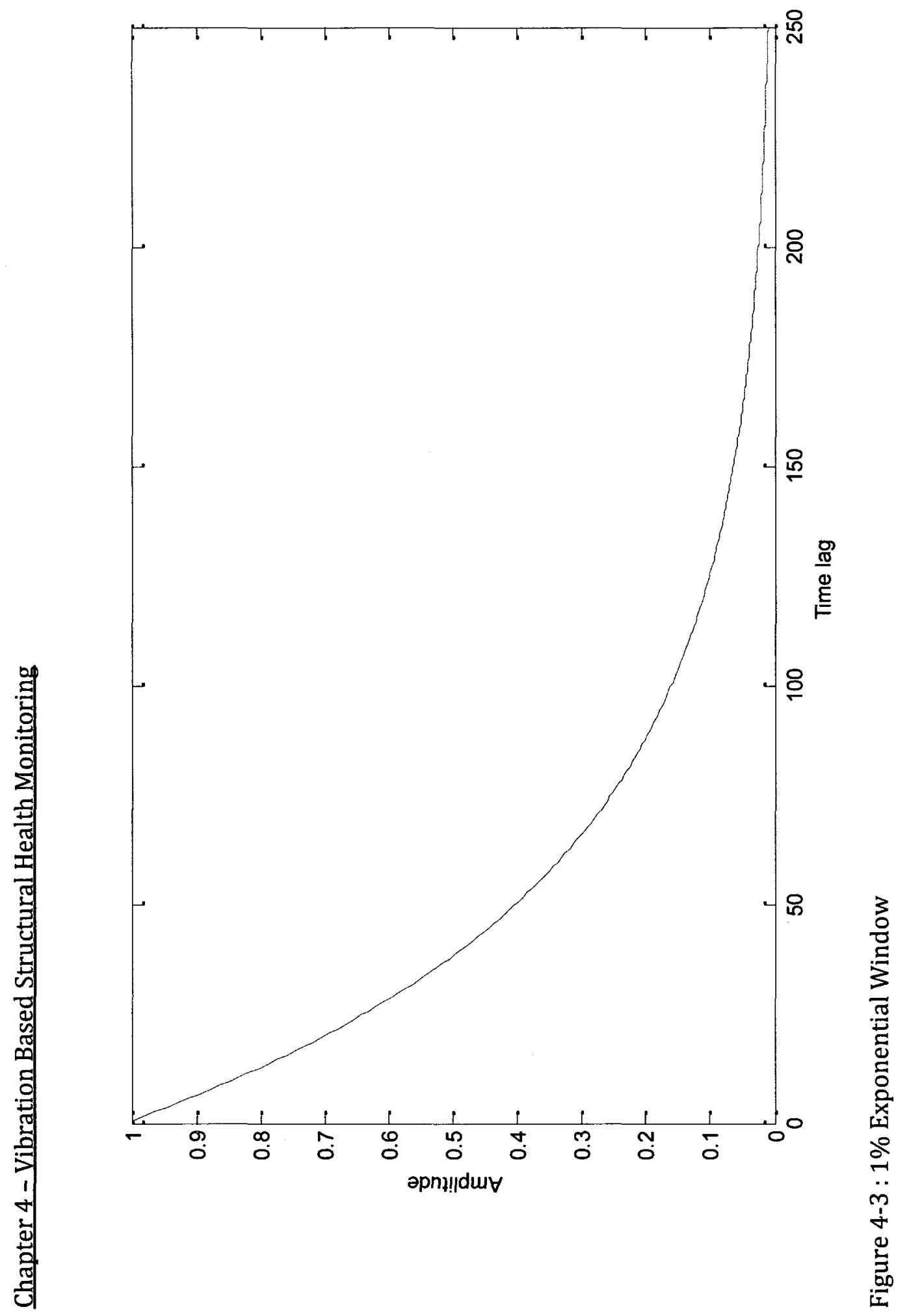


$\bowtie$

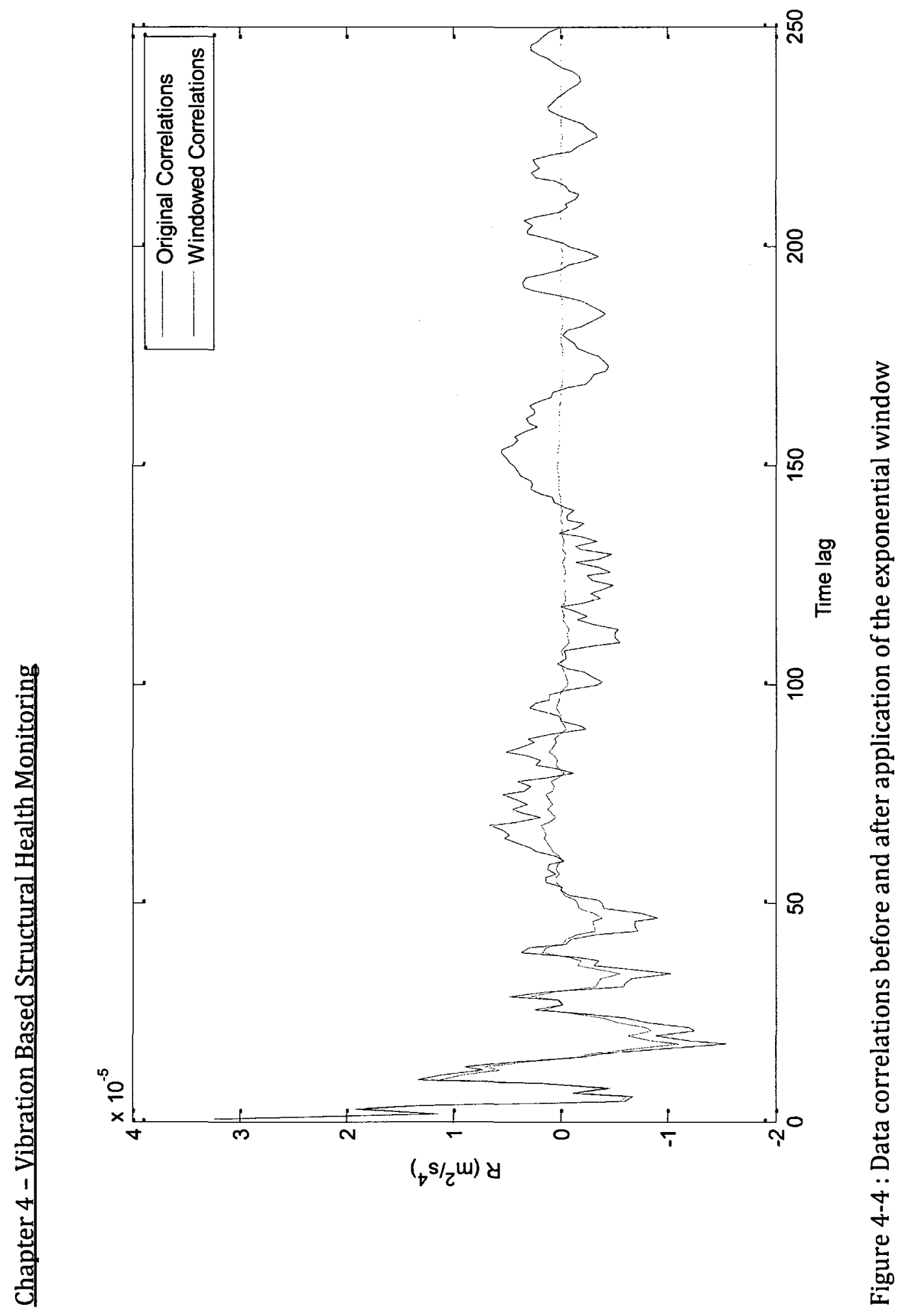




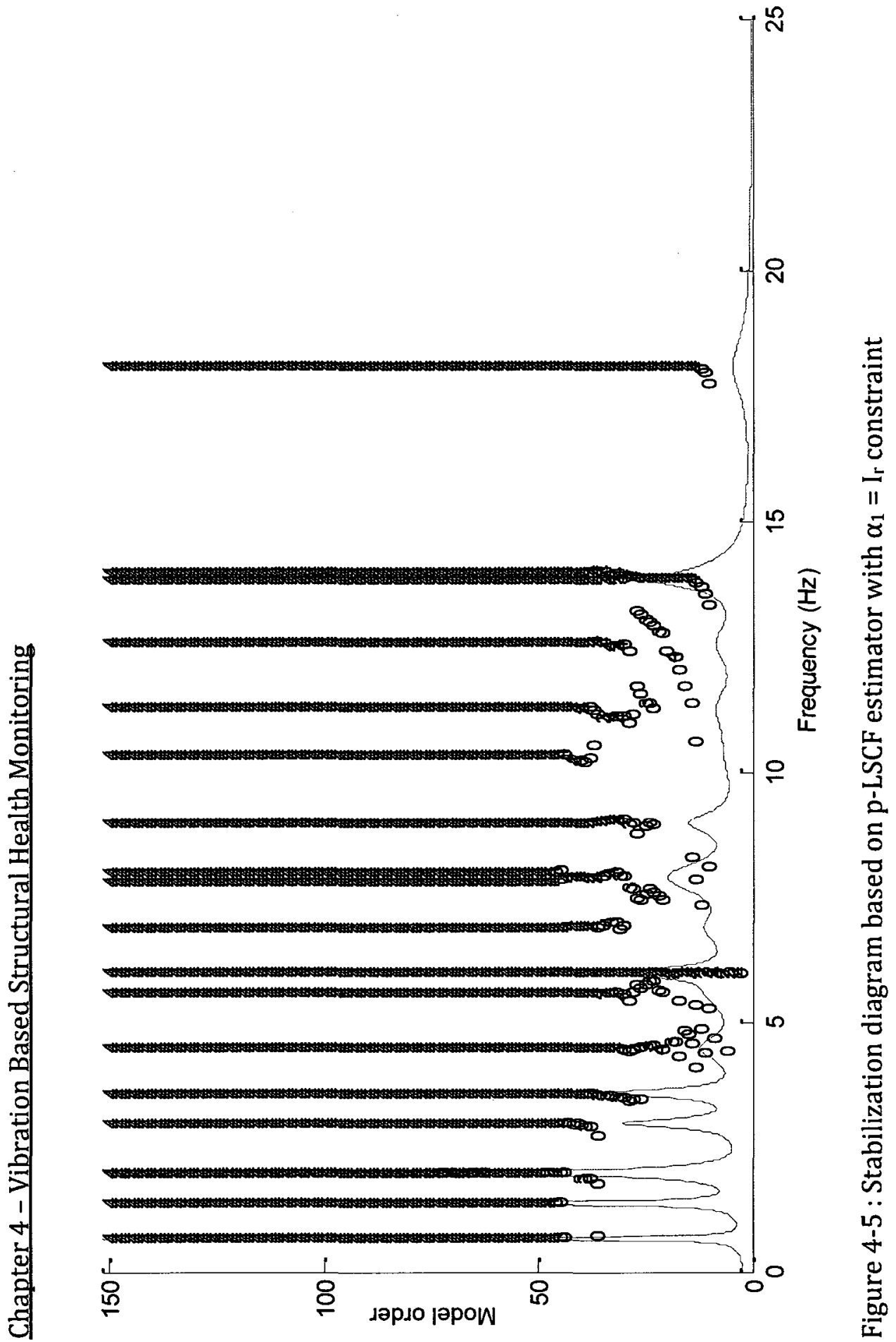




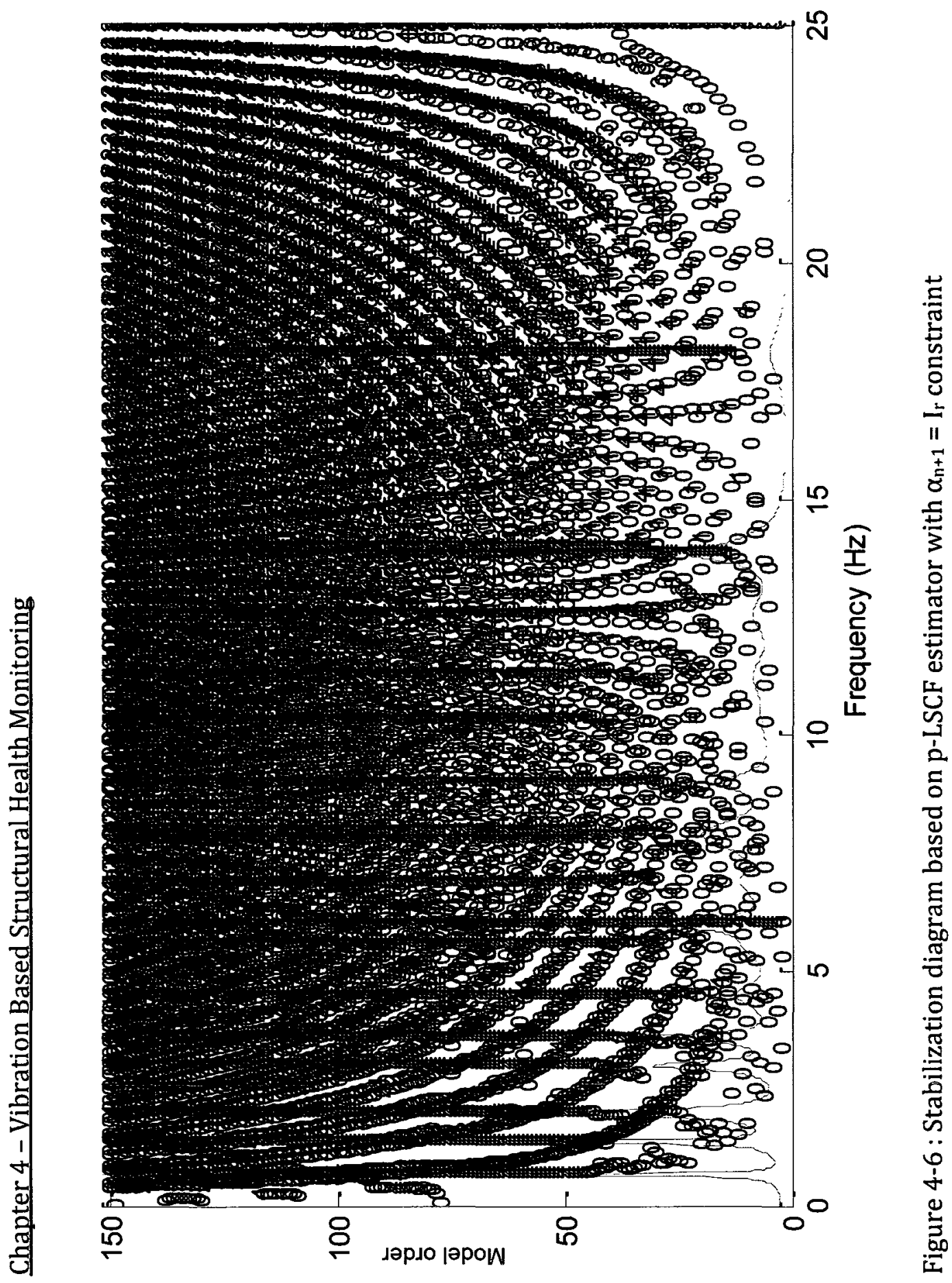




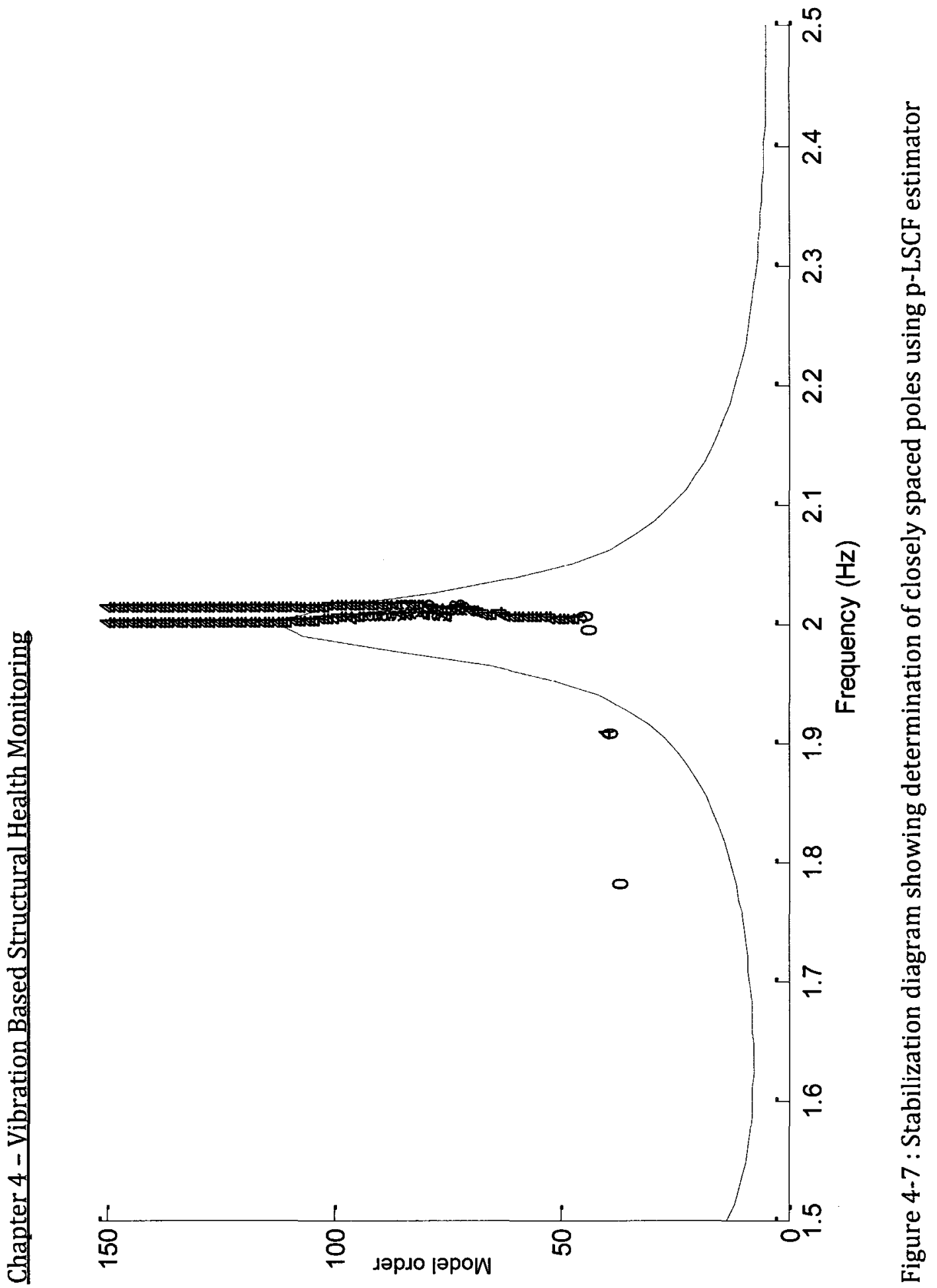




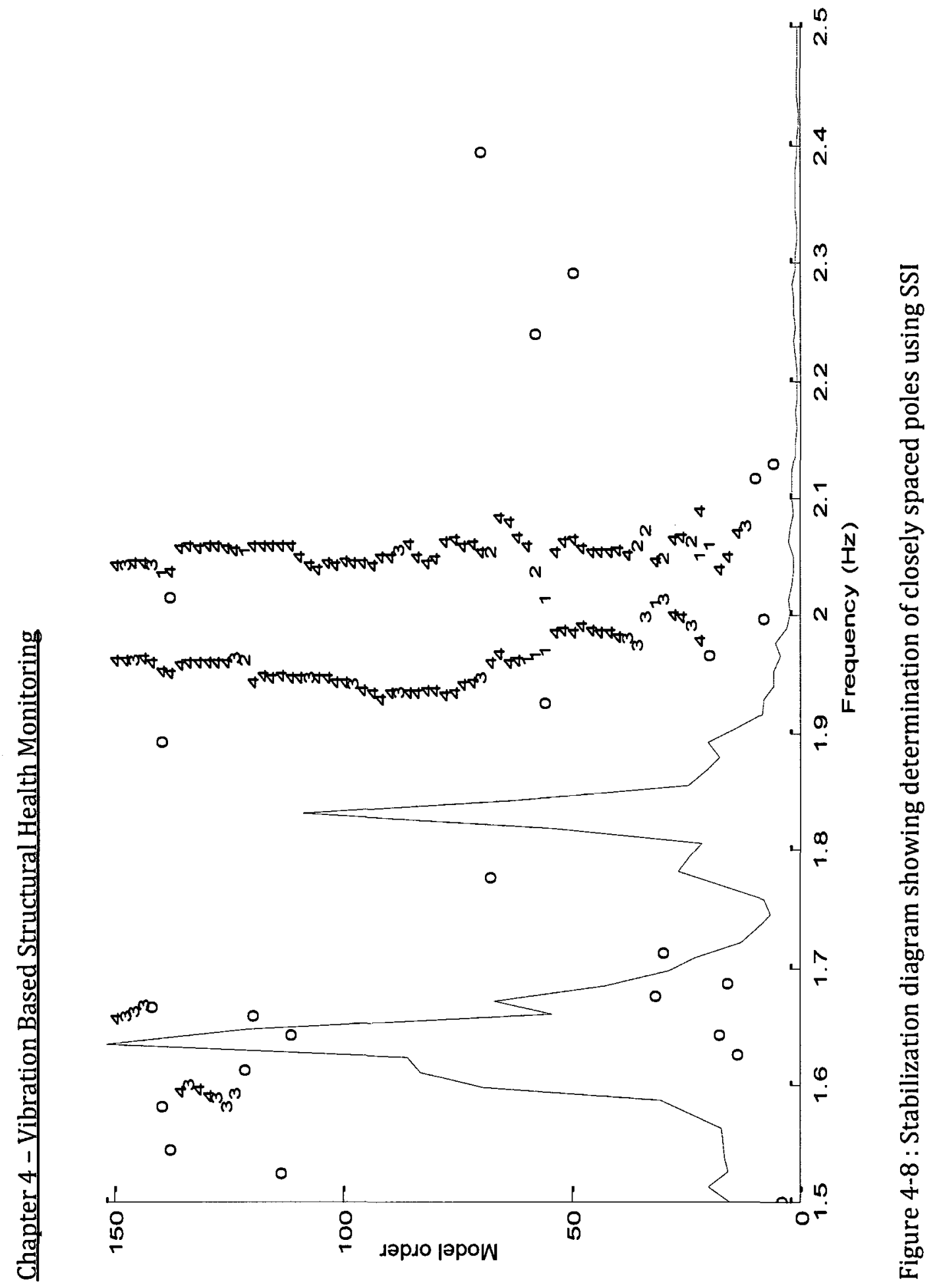




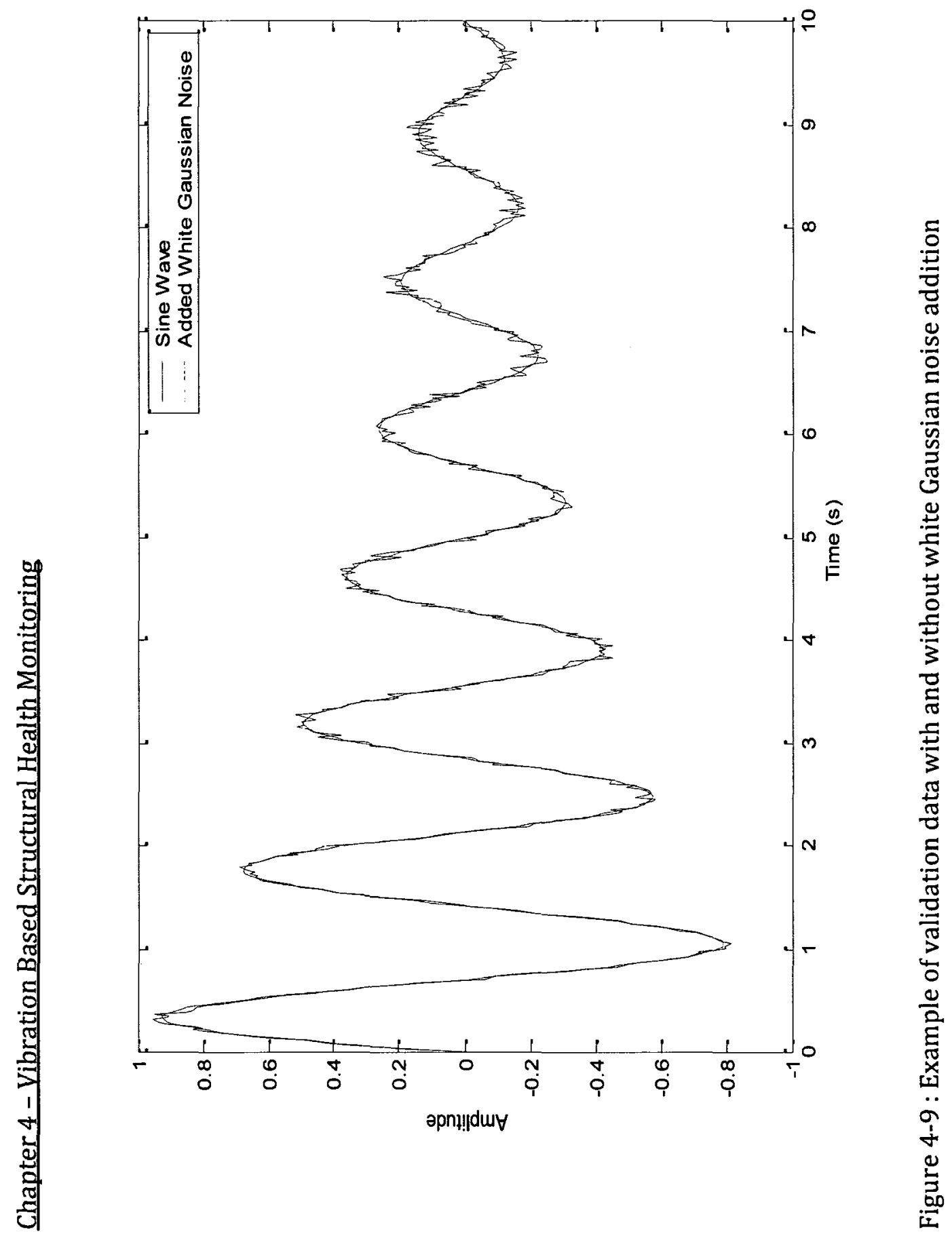




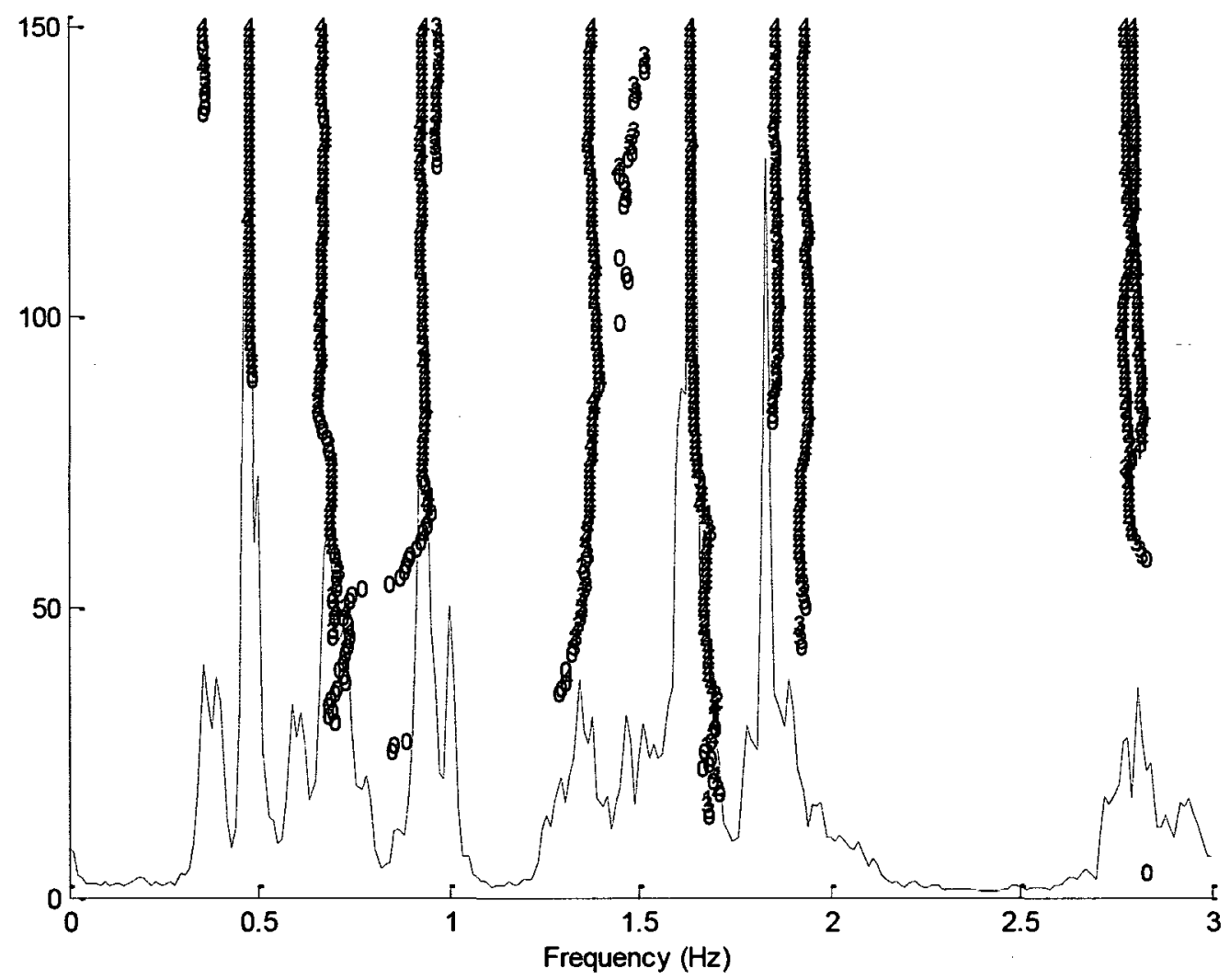

Figure 4-10 : Stabilization diagram generated by p-LSCF estimator 


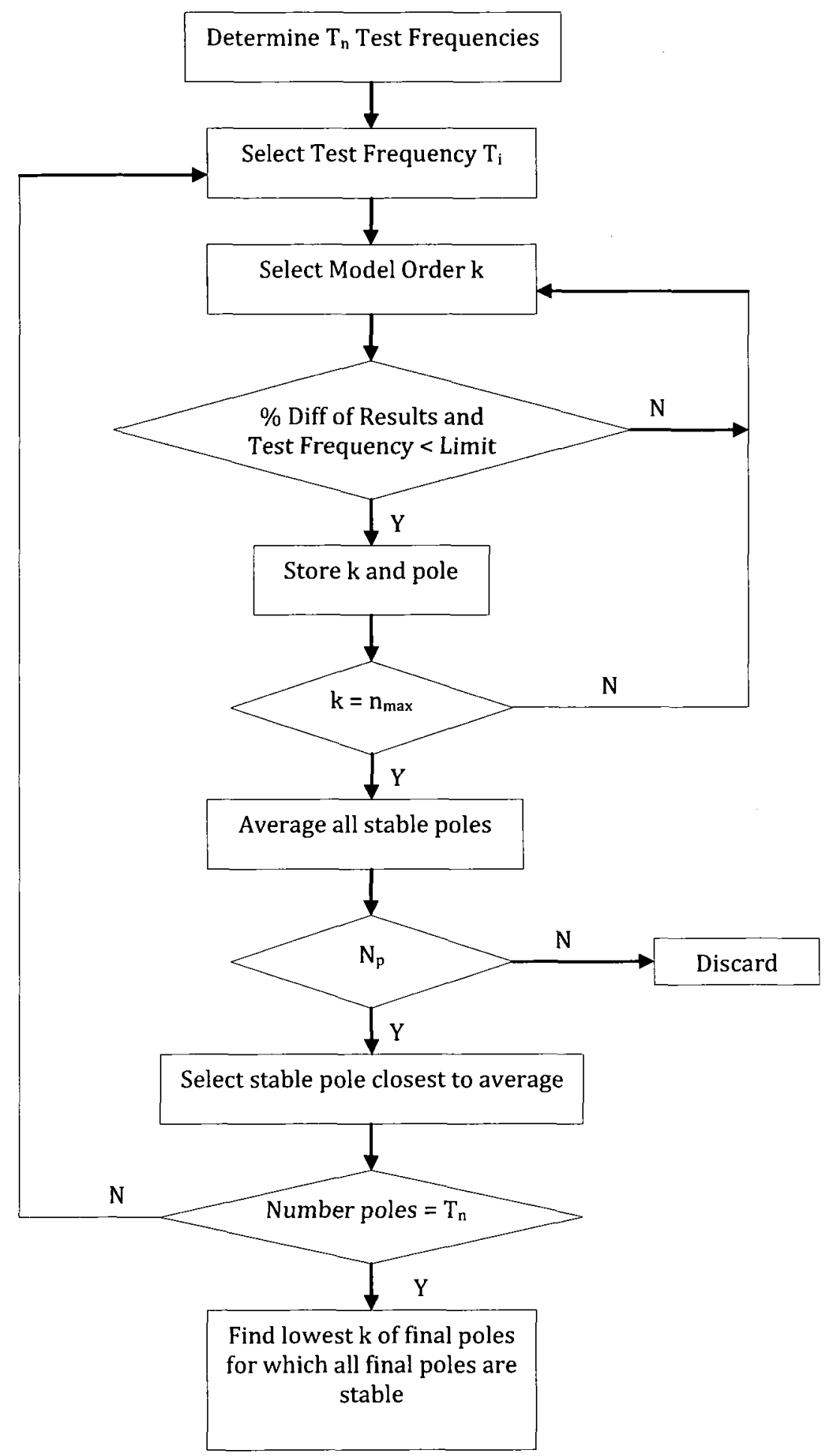

Figure 4-11: Automated pole selection procedure 

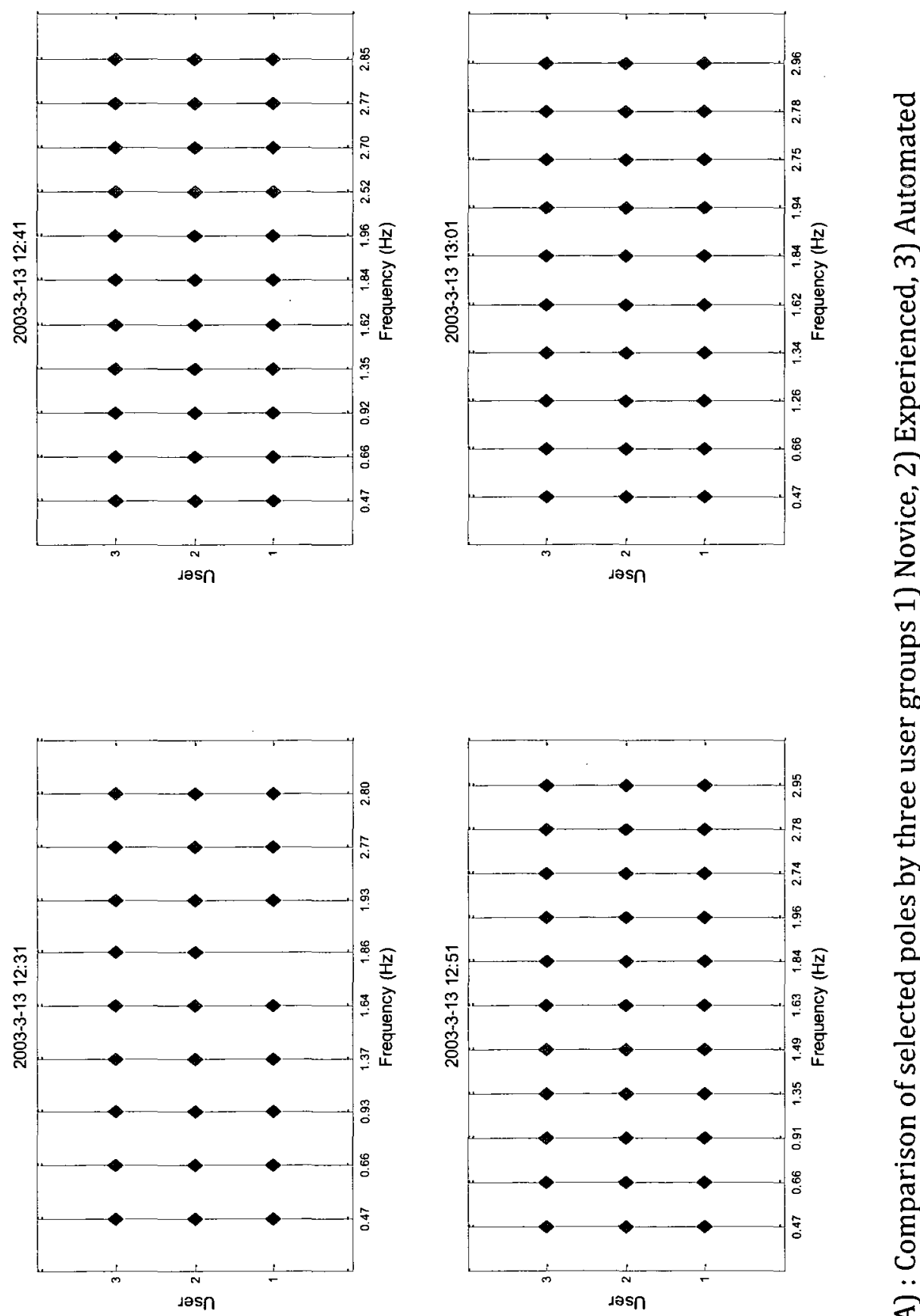

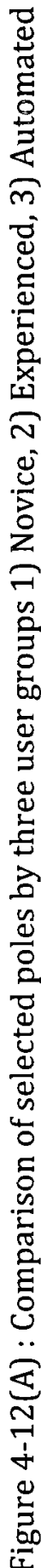



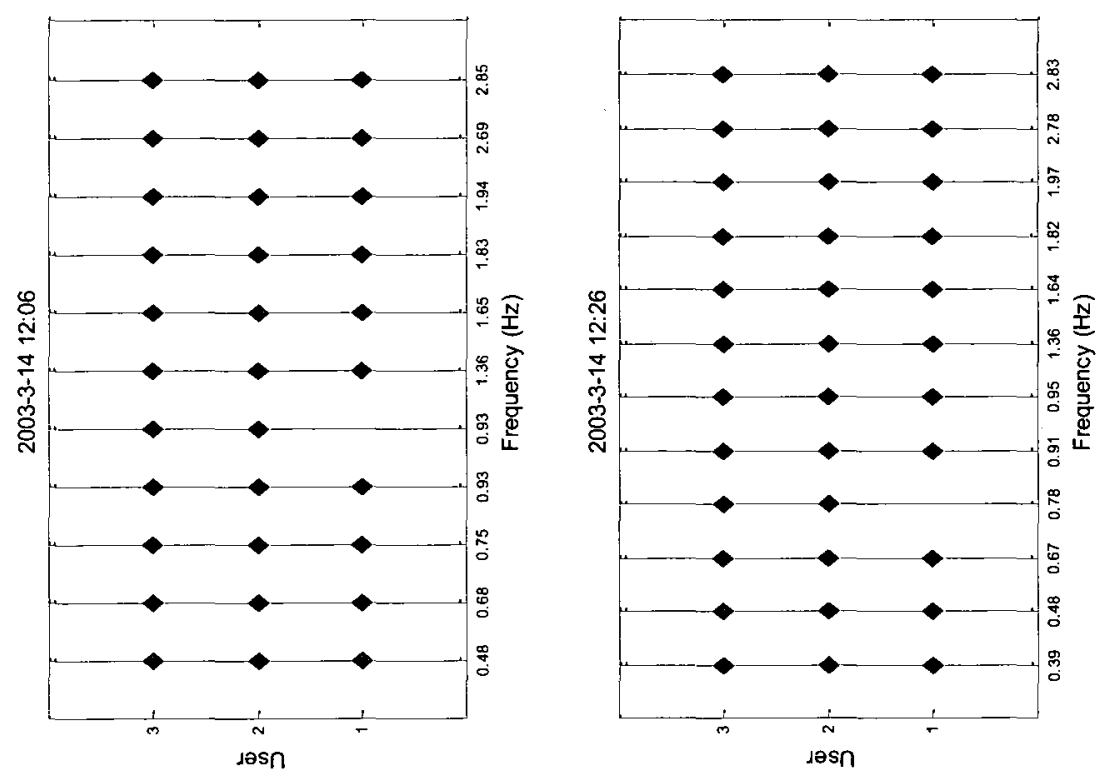

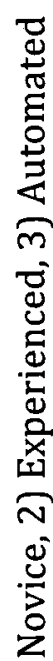
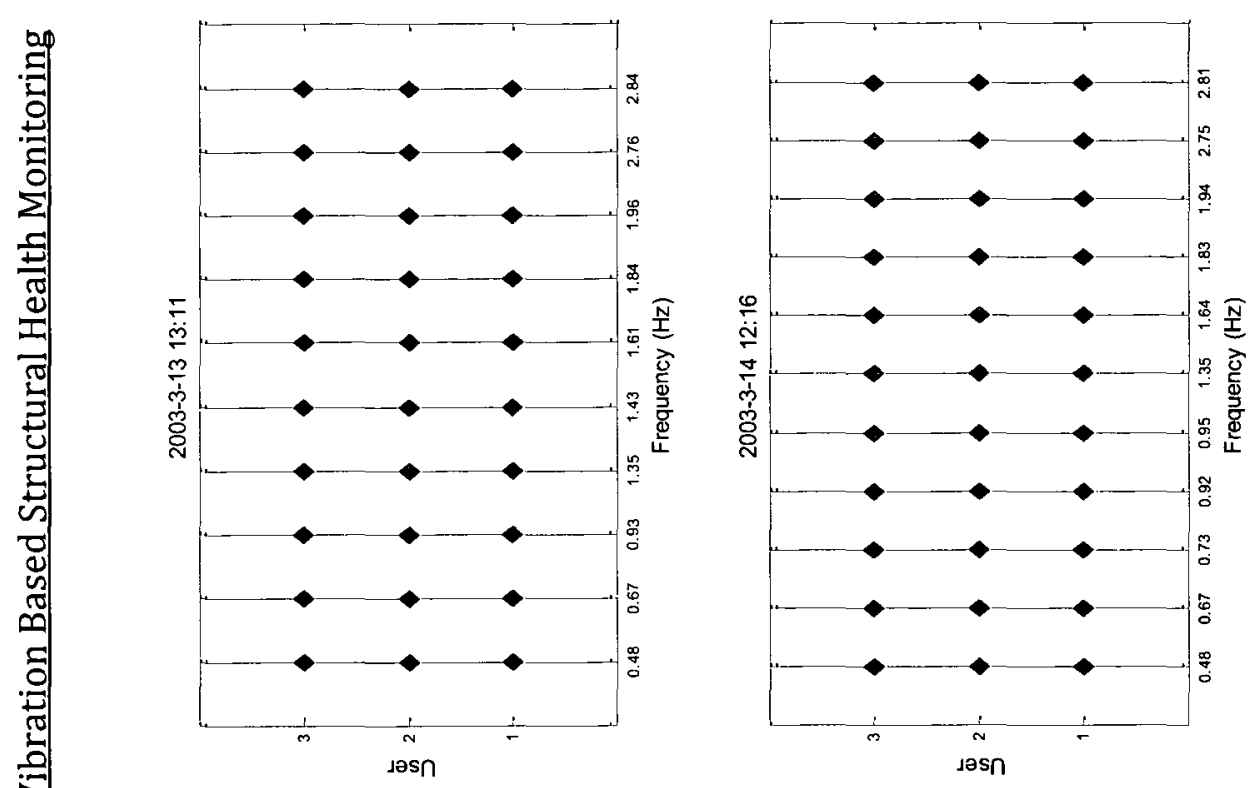


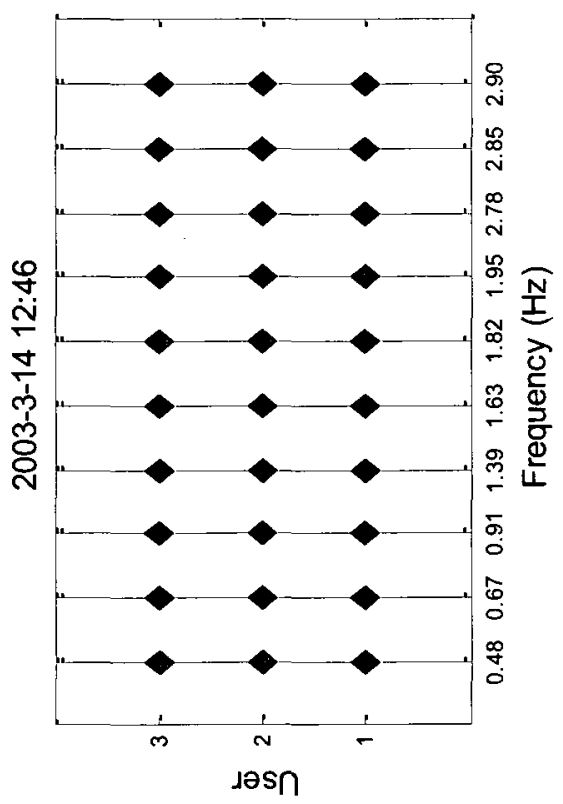

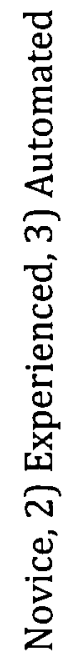

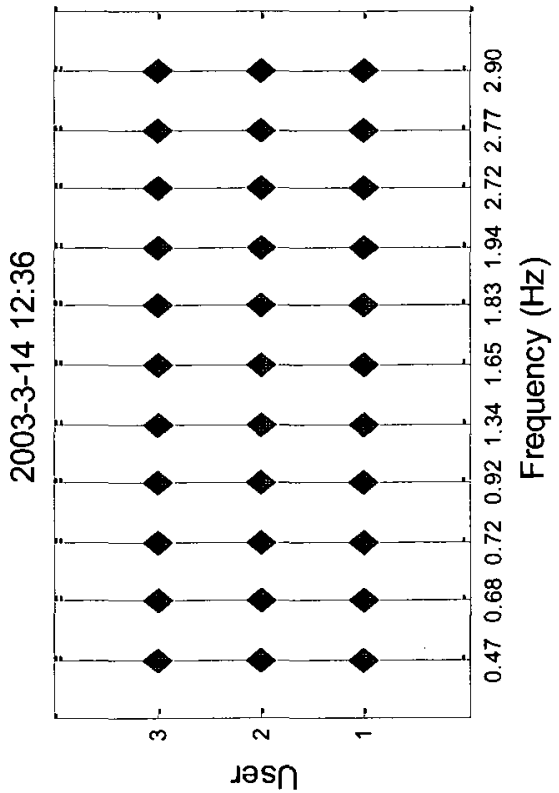

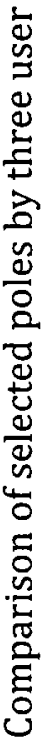

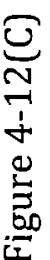




\section{Chapter 5 - Variability of}

\section{Monitoring Data}

\subsection{Introduction}

The basic premise of vibration based structural health monitoring (VBSHM) is that any changes in the vibration properties of a structure are due to some change in the physical conditions of the structure which may be manifested as deterioration or damage of the structure. This premise is valid in theory as evident from basic structural dynamic properties. In practice however, there are several factors which may affect the reliability of applying the vibration based structural health 
Chapter 5 - Variability of Monitoring Data

monitoring principle in practical monitoring applications in the field. Because of the influence of noise and uncertainties in the recorded data, there can be significant variations in the extracted vibration properties from the system identification results. To determine the viability of the VBSHM technique as an alternative approach to manual visual inspection for structural condition assessment, the variability characteristics of the extracted vibration properties from measured vibration response data must be quantified. It is important to properly attribute any identifiable variations in vibration properties of a monitored structure to the correct sources, as related to variation in the operational conditions of the structure or to deterioration or damage. In early phases of this study, Londono (2006) has classified the major sources of variability into two main categories:

1) Modeling, measurement and computational

2) Environmental and loading fluctuations

Variability associated with Category 1 does not affect the vibration properties of the real structure, simply the extracted values from the measured vibration data. On the other hand, Category 2 variability may affect the actual vibration properties of the structure, along with the extracted values.

Londono (2006) further divides the sources of variability in Category 1 into the following sections:

a) Deviations from modeling assumptions.

Measured response data most likely deviate from the model assumptions in the system identification process. Examples of the assumptions which 
may exhibit deviations are linearity of the model, stationarity of the data, or whiteness of the input signal.

b) Measurement uncertainties.

These uncertainties are found in every electronic monitoring system and can be attributed to the equipment utilized in recording the measurement data, such as sensors, wiring, and data-loggers. While a properly configured and maintained system can minimize these uncertainties, they cannot be eliminated completely.

c) Computational uncertainties.

This source of variability in the monitoring data can be attributed to several factors, including the use of finite datasets which may affect the accuracy in the calculation of data correlations due to the varying lengths of the recorded data. As well, noise may be induced in the data by such processes as filtering and re-sampling. Also, it has been shown that significant variations in extracted values can arise depending upon the selected system identification technique (Peeters \& Ventura, 2003).

Similar to Category 1, Londono (2006) divides Category 2 sources of variability into the following:

a) Environmental fluctuations.

Variations in temperature have been shown to greatly affect the extracted modal frequencies of a system (Londono, 2006), (Peeters et al. 2001). This effect may be attributed to the changes in material properties due to 
thermal variations. For example a variation in the elastic module of concrete due to temperature affects the stiffness of a structure. Of importance for bridges over ice-covered waters is the effect that the presence of ice surrounding piers may contribute to the alteration of boundary conditions due to the restraining action of the ice floes on the pier movement.

b) Different loading scenarios.

Data collected under the same environmental conditions may display some variations in extracted vibration properties due to fluctuations in the loading scenarios, which may differentiate due to multiple sources.

- The excited frequencies of a structure may vary based upon the excitation source of the system (Peeters \& Ventura, 2003). This is of particular significance in an output-only system where significantly different excitation sources such as wind, traffic, and ice loading are present.

- The amplitude of the excitation source has been found to affect the extracted vibration properties of a structure (Ndambi, et al., 2000).

- The presence of heavy traffic loads can affect the mass of the structure (Farrar et al. 1997). 


\subsection{Baseline Variability}

Baseline variability can be attributed to the variability associated with category one sources of variability, i.e. modeling assumptions, measurement uncertainties, and computational uncertainties. If data could be collected under the same environmental conditions and loading scenarios then the only variability present would be a result of baseline variability, assuming that the structure's condition remains constant. In the study to understand the general variability of monitoring data it is best to first determine the baseline variability. This can be achieved by analyzing data sets which are recorded during a short period of time, during which the environmental conditions and loading scenarios can be considered constant, along with the condition of the structure. For this study, ten datasets from the Confederation Bridge have been analyzed using the p-LSCF estimator and the automated pole selection algorithm. The datasets consist of ten minute vibration response measurements from fifteen accelerometers located at locations 5 to 9 as shown in Figure 5-1. Figure 5-2 shows that the average temperature of the concrete at the instrumented section of the bridge and the wind speed at the bridge site are relatively consistent for the datasets. The range of average concrete temperature is $-2.7^{\circ} \mathrm{C}$ to $-1.4^{\circ} \mathrm{C}$, while the average wind speed range is $7.2 \mathrm{~m} / \mathrm{s}$ to $14.8 \mathrm{~m} / \mathrm{s}$. The range of these values, $1.3^{\circ} \mathrm{C}$ and $7.6 \mathrm{~m} / \mathrm{s}$ are reasonable when compared to the yearly variations of $45^{\circ} \mathrm{C}$ and $30 \mathrm{~m} / \mathrm{s}$ for the conditions. Figure $5-3$ shows the time 
history of the ten datasets utilized in the study, while Figure 5-4 displays the average normalized power spectral density of the datasets, both of which show the relative consistency of the environmental and loading scenarios with respect to the datasets.

\subsection{Baseline Variability Results}

The results of the baseline variability study are shown in Table 5-1 and Figure 5-5 and Figure 5-6. In Figure 5-5 and Figure 5-6 the frequency and damping ratios are normalized by their mean values respectively. It can be seen from Figure 5-5 that the modal frequencies are typically within $\pm 1.5 \%$, showing excellent consistency. The average standard deviation is $0.84 \%$ of the mean, which is increased due to large values attributed to the last two frequencies, $2.7479 \mathrm{~Hz}$ and $2.8073 \mathrm{~Hz}$. These larger than normal standard deviations can be attributed to the closeness of the poles, which can result in the incorrect placement of a selected pole into a predetermined frequency bin. This difficulty can be explained by the inability of the p-LSCF estimator to determine the mode shape of a selected pole. These results show an increase in the baseline variability when compared to the results provided by the stochastic subspace identification technique. The average standard deviation is $0.51 \%$ of the mean based on the stochastic subspace identification method (Londono, 2006). 
Unlike the modal frequencies, the modal damping ratios are quite scattered as seen in Figure 5-6, where the normalized values typically range from 0.4 to 1.5 , a stark contrast to the relatively consistent values of the modal frequencies. The average standard deviation is $36 \%$ of the mean, showing substantial variance in the results. These results demonstrate the inability of modal damping ratios to be used for VBSHM due to the significant variability associated with extracted values. This variability can be attributed to the complex nature of the damping phenomenon.

\subsection{Environmental and Loading Variability}

The uncontrolled field conditions associated with civil engineering structures results in uncertainties arising from environmental conditions and loading scenarios. These uncertainties are unavoidable and must be quantified in order to obtain a measure of the general variability of a monitoring system. For this study, twenty-two datasets, from a six month period, are collected and analyzed using the p-LSCF estimator and automated pole selection procedure outline in Chapter 4. These datasets are selected based on the average temperature of the concrete, which were determined using 156 thermocouples installed as part of the Confederation Bridge monitoring project, with one dataset present for each two degree increment of the typical yearly range of $-20^{\circ} \mathrm{C}$ to $25^{\circ} \mathrm{C}$. Figure $5-7$ shows the 
time histories of the selected datasets, while Figure 5-8 displays the average normalized power spectral density of the datasets. These two sets of figures demonstrate the variations in the measured response data due to the different environmental conditions and loading scenarios.

\subsection{Environmental and Loading Variability Results}

The results of the environmental variability study are shown in Table 5-2, Figure 5-9, and Figure 5-10. These results show an obvious increase in the variability of the extracted modal frequencies. The average standard deviation of the mean frequencies has increased to $1.50 \%$ of the mean, an increase of roughly 1.8 times over the baseline variability data, showing that the environmental and loading scenarios fluctuations contribute nearly half of the general variability. Once again there is an increase in the variability when compared to the stochastic subspace identification technique. Londono (2006) determined the average standard deviation of the vibration frequencies to be $1.11 \%$ of the mean for environmental and loading scenario variability. However the increase in variability of roughly 1.8 times the baseline variability is similar to that determined by Londono (2006).

Contrary to the modal frequencies, the damping ratios remain relatively consistent compared to those extracted from the baseline variability data, with a standard deviation of $39 \%$ of the mean and $36 \%$ of the mean respectively. These 
results show no correlation between the damping ratios and the environmental conditions and loading scenarios, which leads us to believe that variability of the damping ratios is a result of the complex energy dissipation of the system.

Figure 5-9 shows a linear trend in the data when one considers that the datasets have been arranged in order of increasing average concrete temperature. This trend can be explained by the increase in temperature resulting in a decrease in the elastic modulus of concrete. This decrease results in an increase in flexibility of the structure, resulting in an increase in the period of a structure. Linear regression has been employed for the determination of possible correlation between average concrete temperature and modal frequency. The results of this regression are shown in Table 5-3. The two lowest modes, $0.4735 \mathrm{~Hz}$ and $0.6694 \mathrm{~Hz}$ show a weak correlation between frequency and average concrete temperature, while the remaining modes show a relatively good correlation, with the $2.7912 \mathrm{~Hz}$ mode showing a relatively strong correlation with a coefficient of determination of 0.78. As a result of this linear regression, the standard error of the frequency has been determined to be $1.03 \%$ of the mean of the frequency. This is a significant reduction from the average standard deviation of $1.50 \%$ of the mean prior to regression. As a result it can be concluded that the elimination of temperature effects on monitoring data can result in almost complete elimination of physical variability, leading to a general variability which approaches the value of baseline variability. 


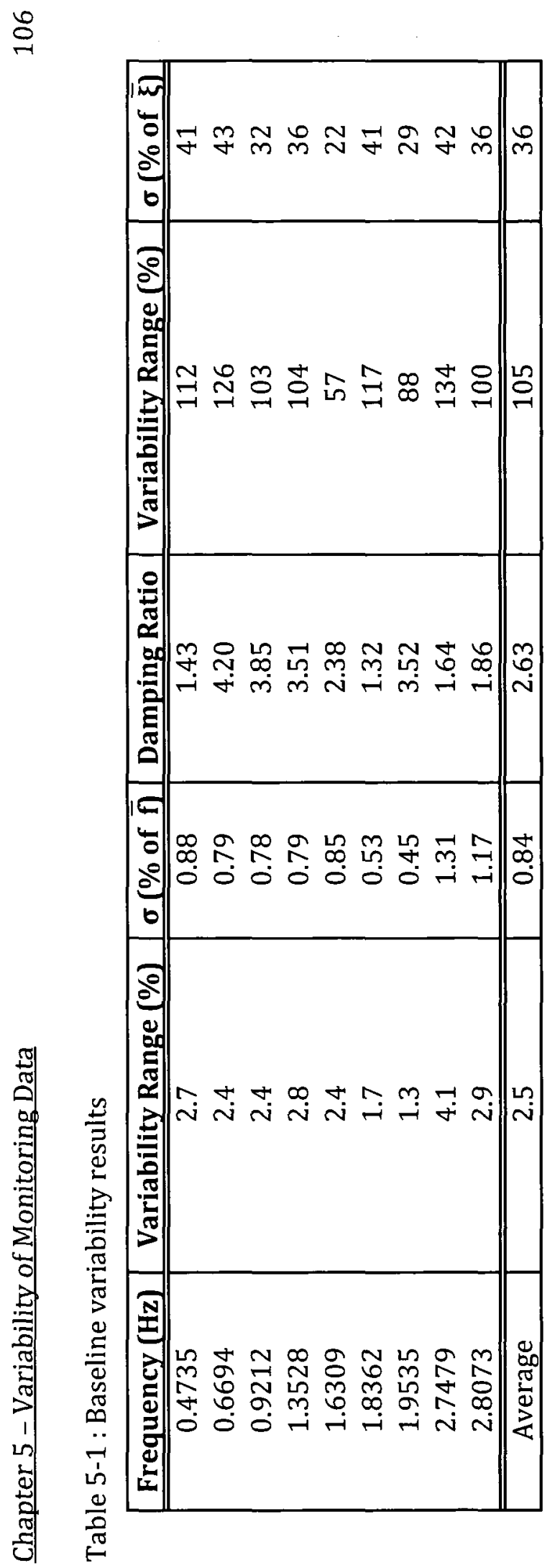




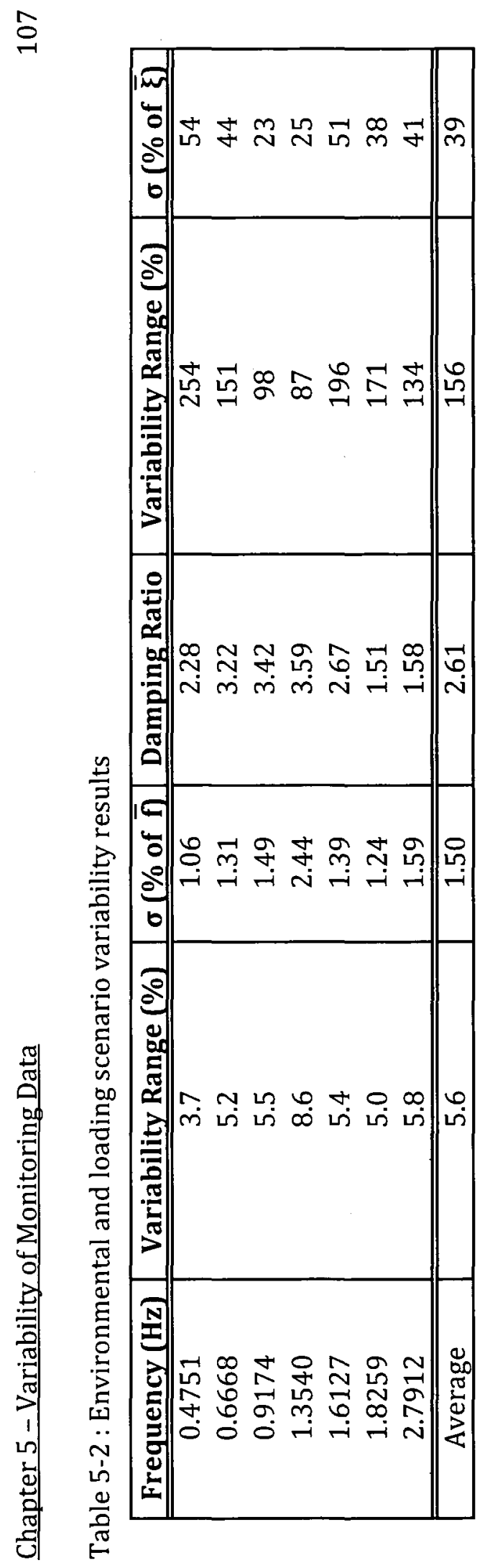




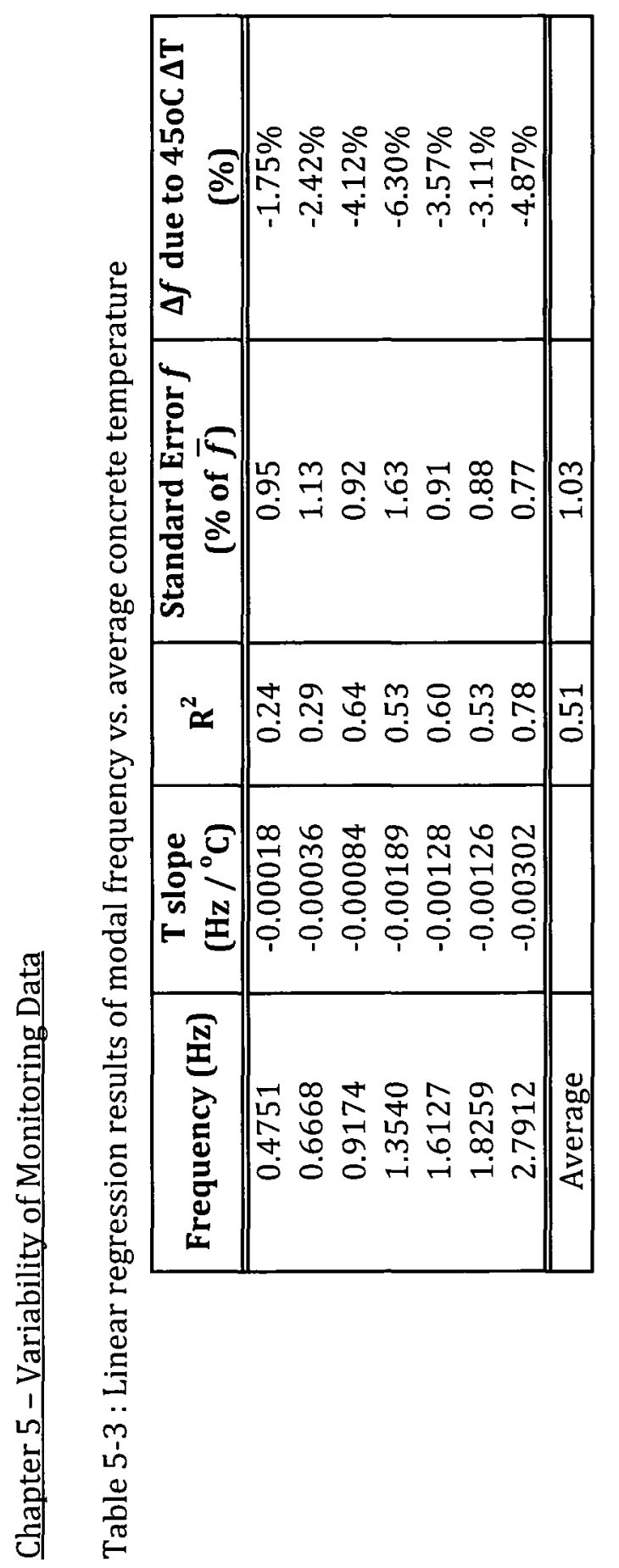




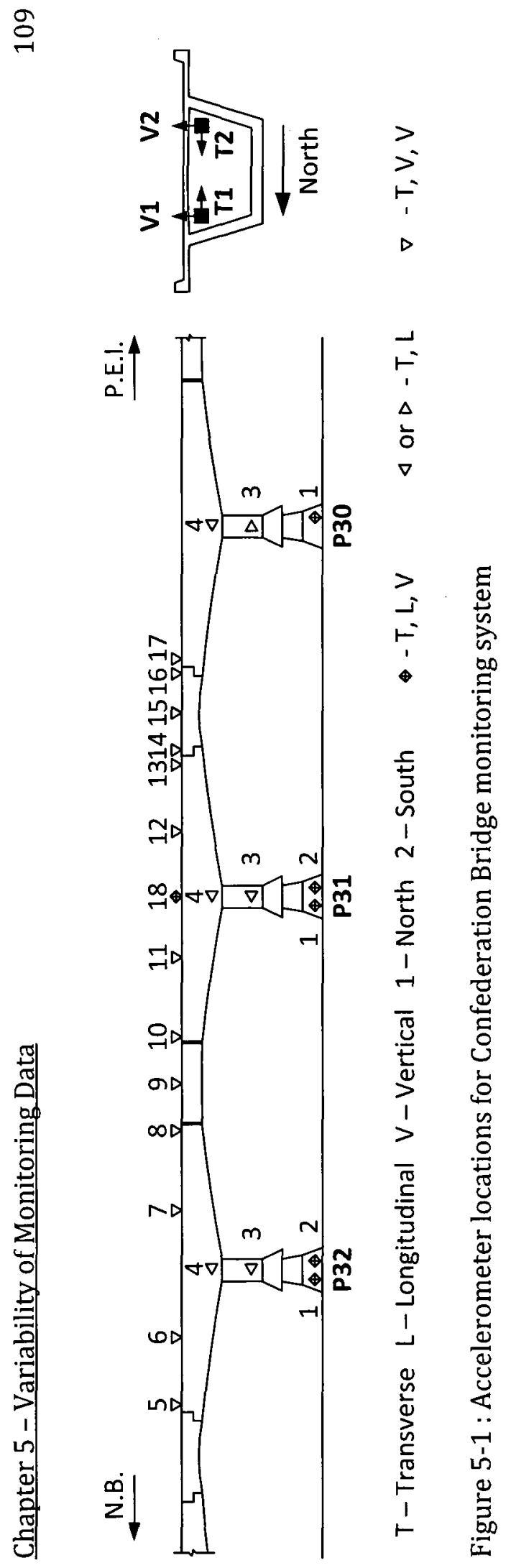




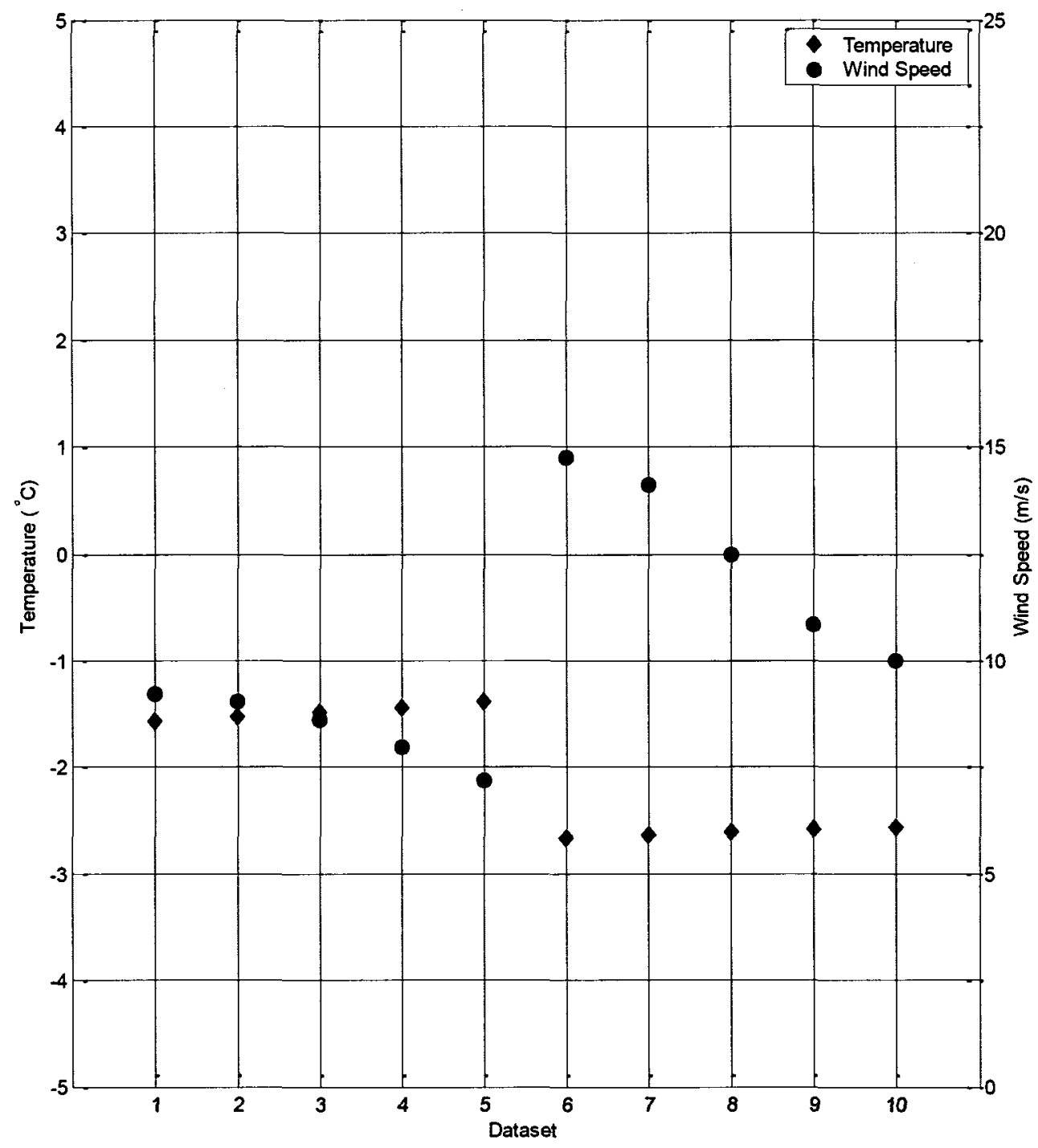

Figure 5-2: Average concrete temperature and average wind speed of baseline variability datasets 
Chapter 5 - Variability of Monitoring Data
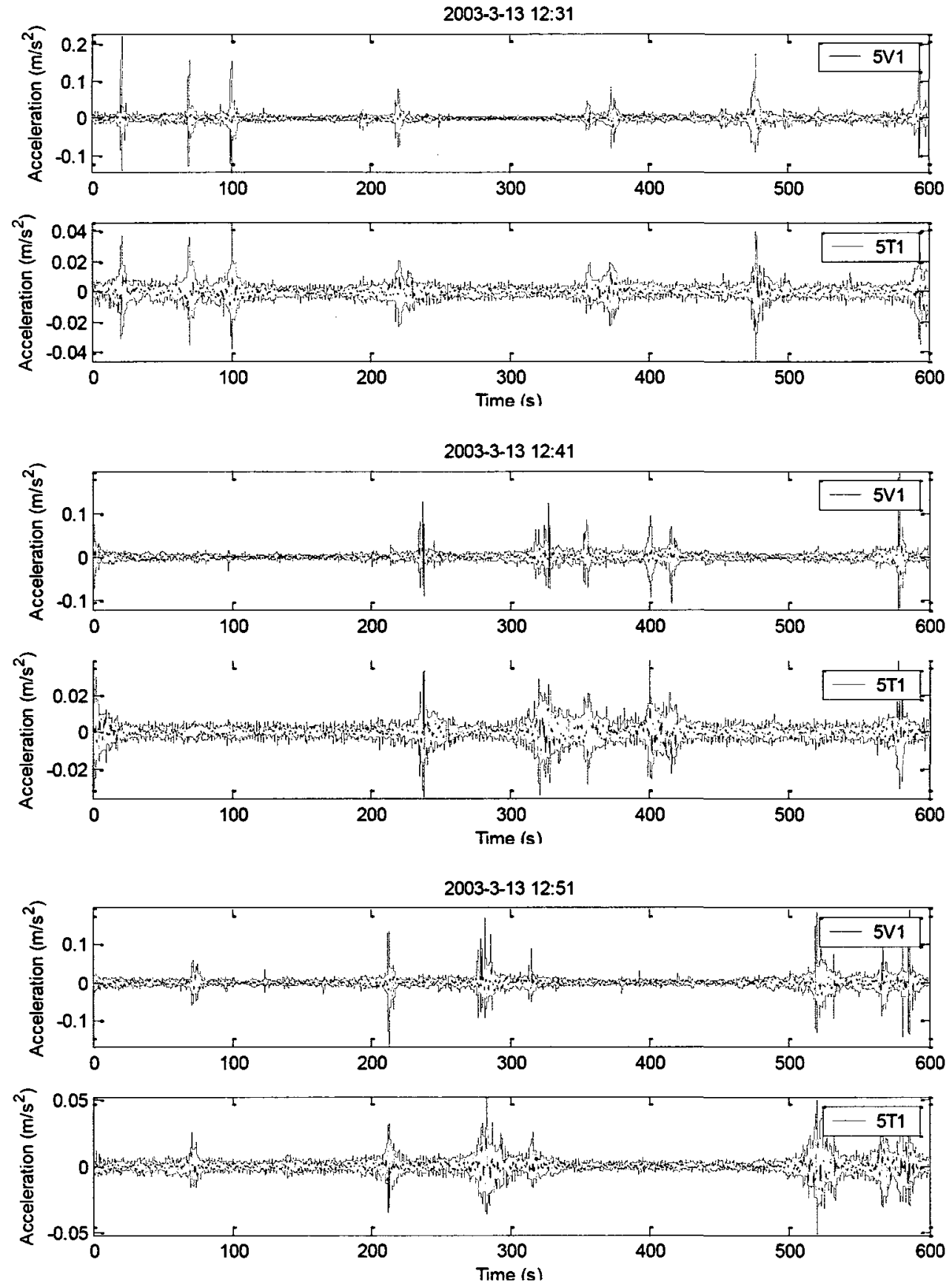

Figure 5-3(A) : Time histories of baseline variability datasets 
Chapter 5 -Variability of Monitoring Data
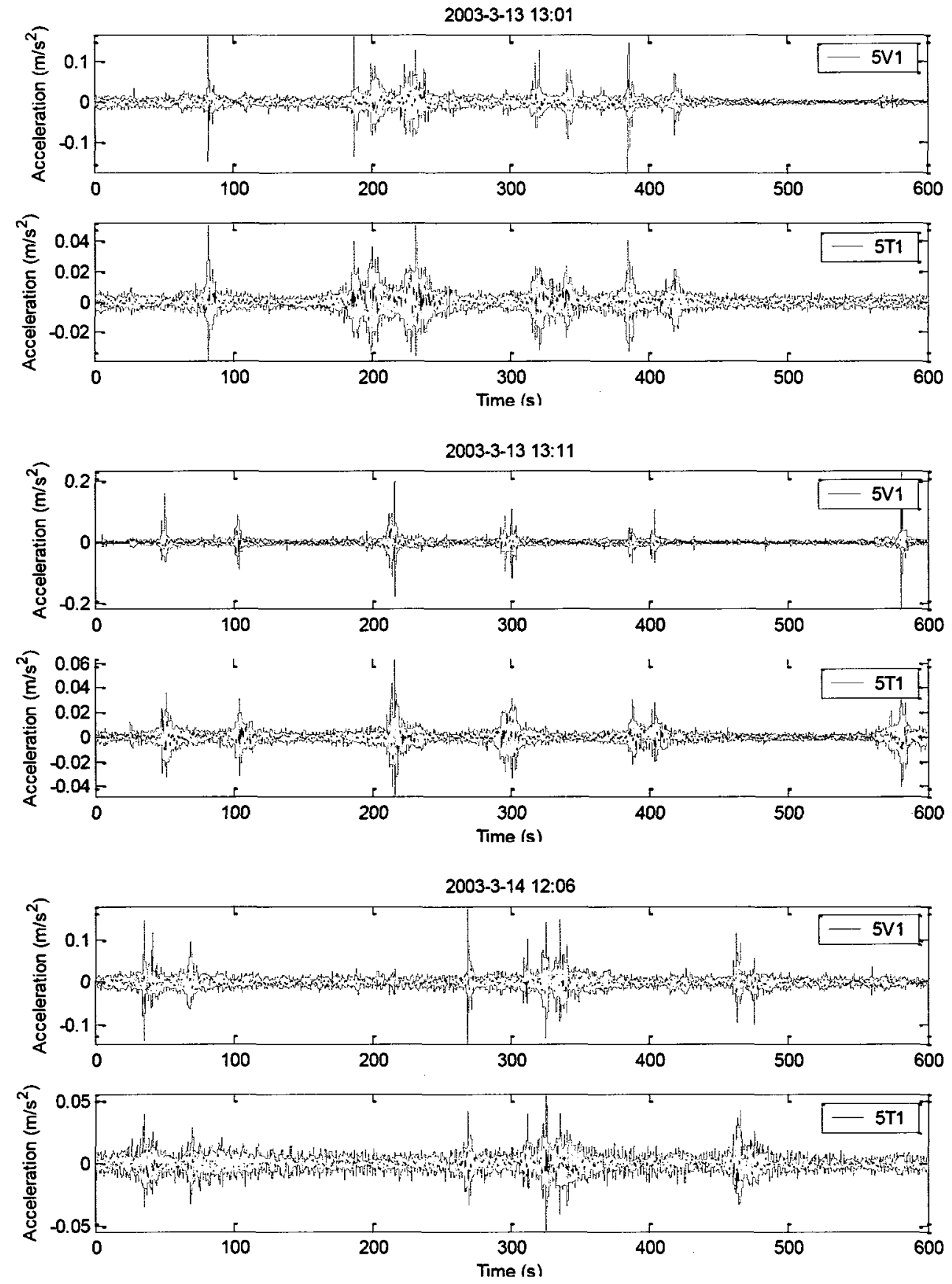

Figure 5-3(B) : Time histories of baseline variability datasets 

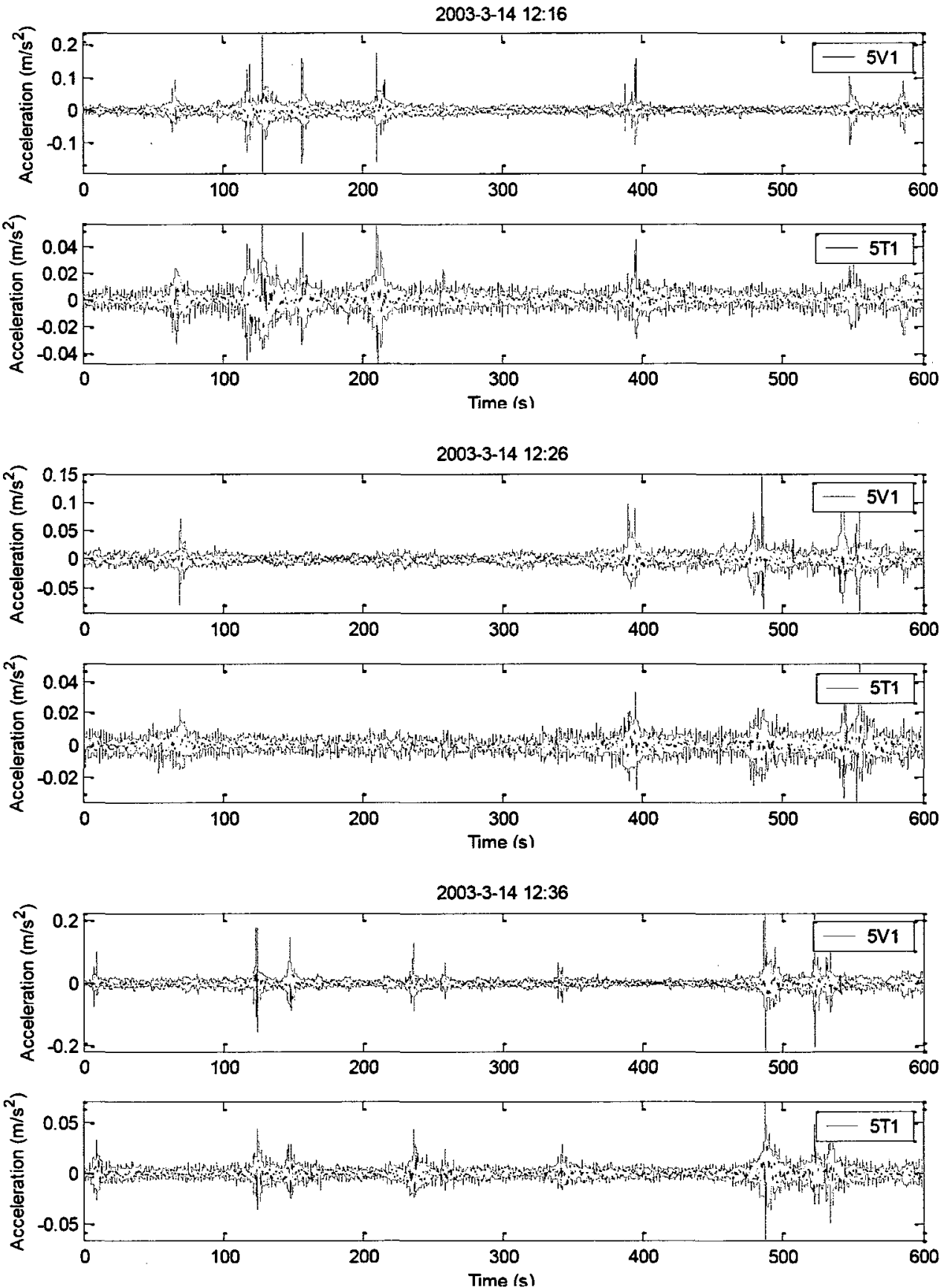

Figure 5-3(C) : Time histories of baseline variability datasets 

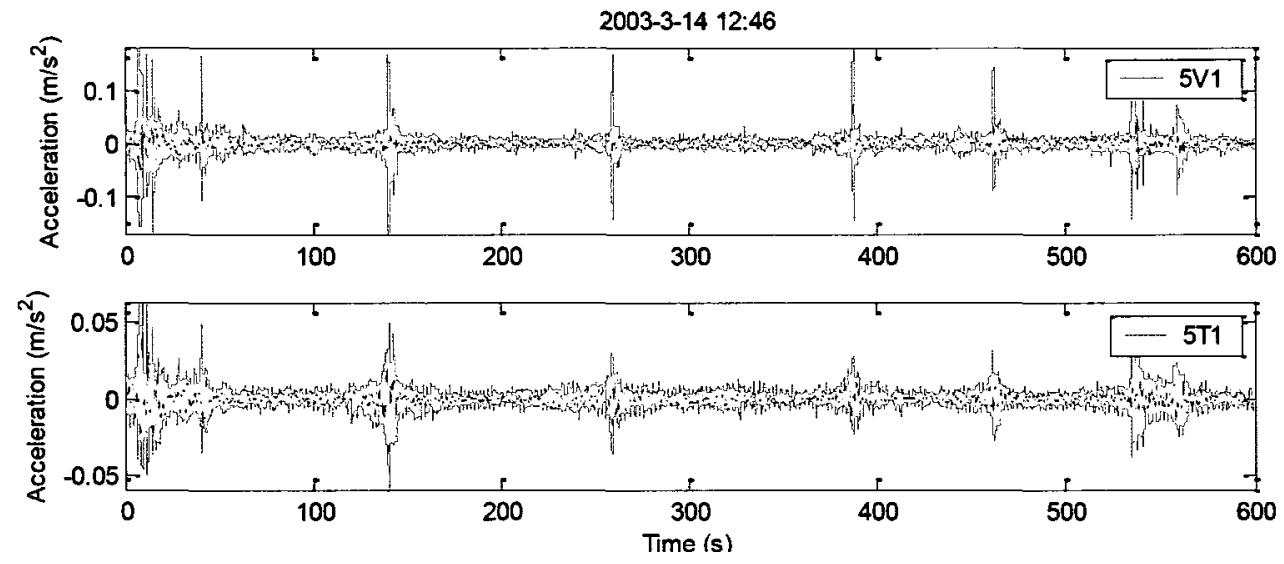

Figure 5-3(D) : Time histories of baseline variability datasets 
록
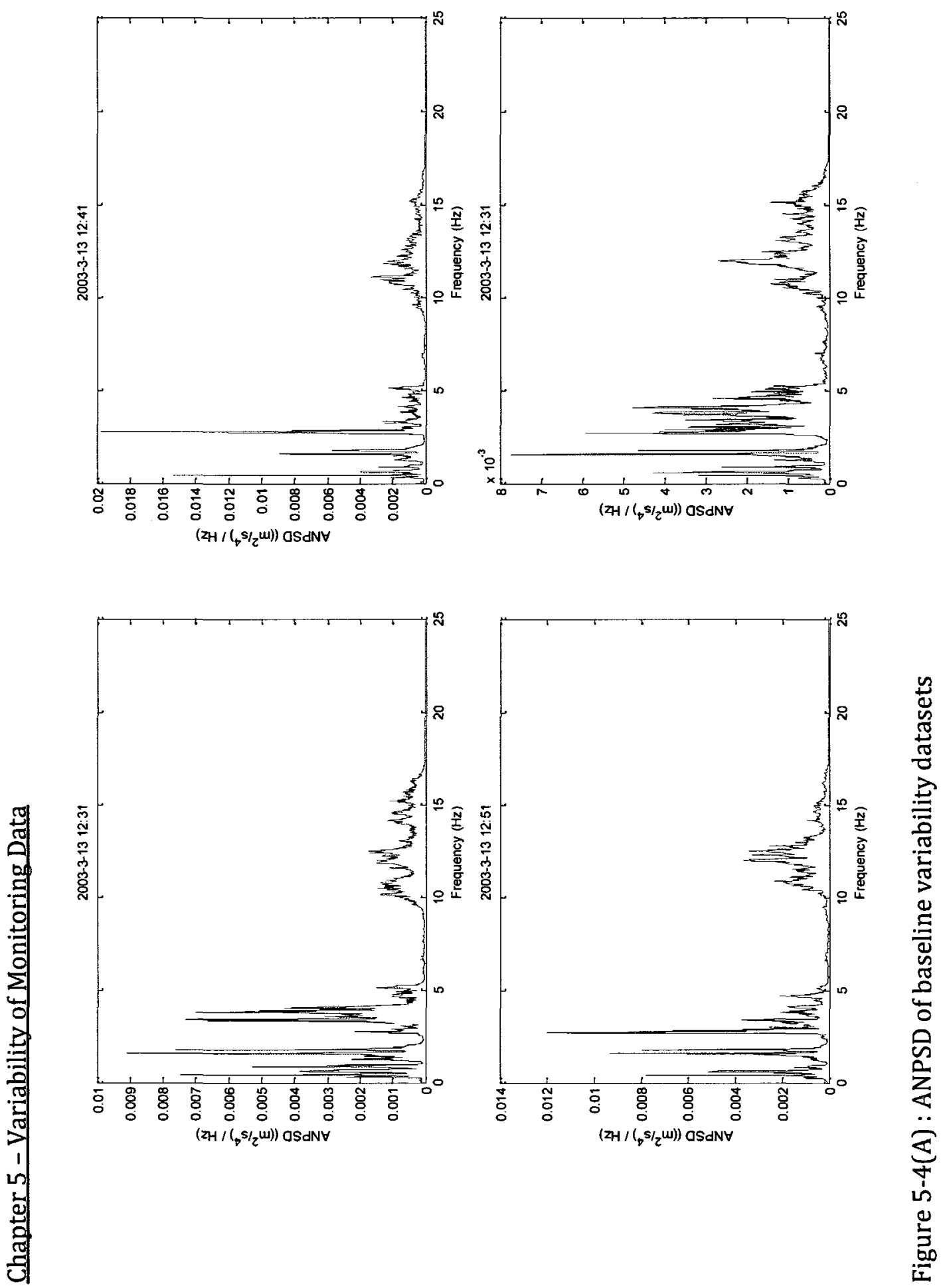
I
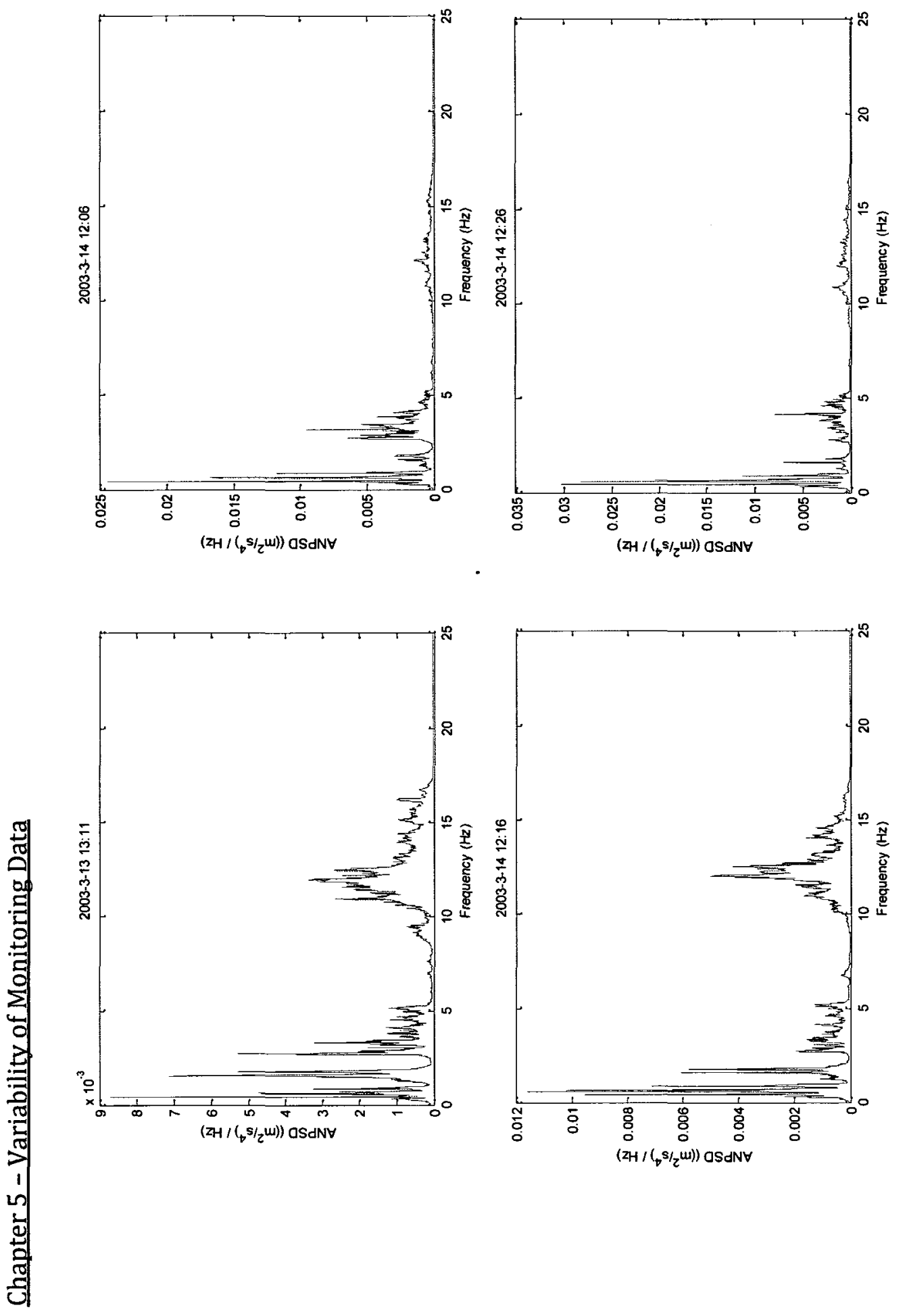

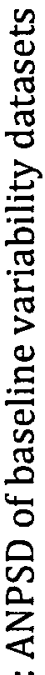

હ્ષ્

苛 
$\exists$
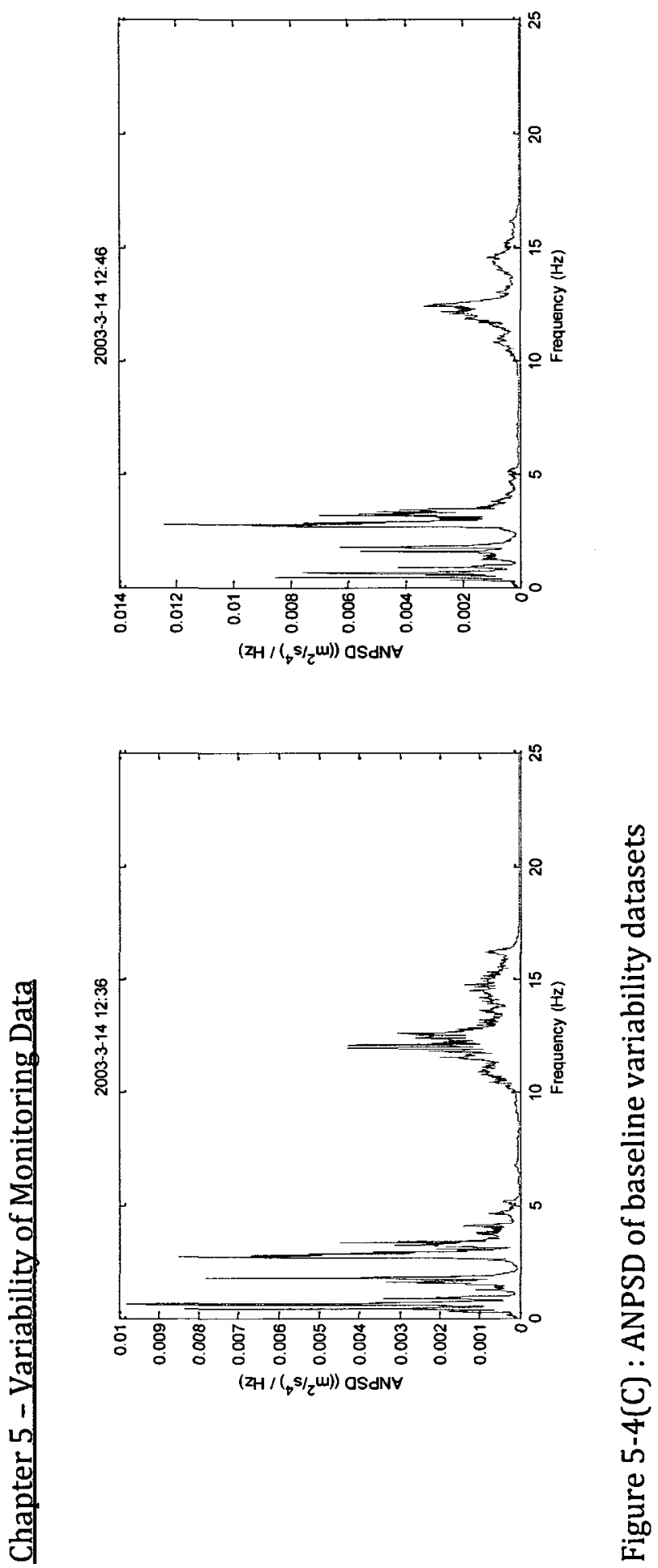

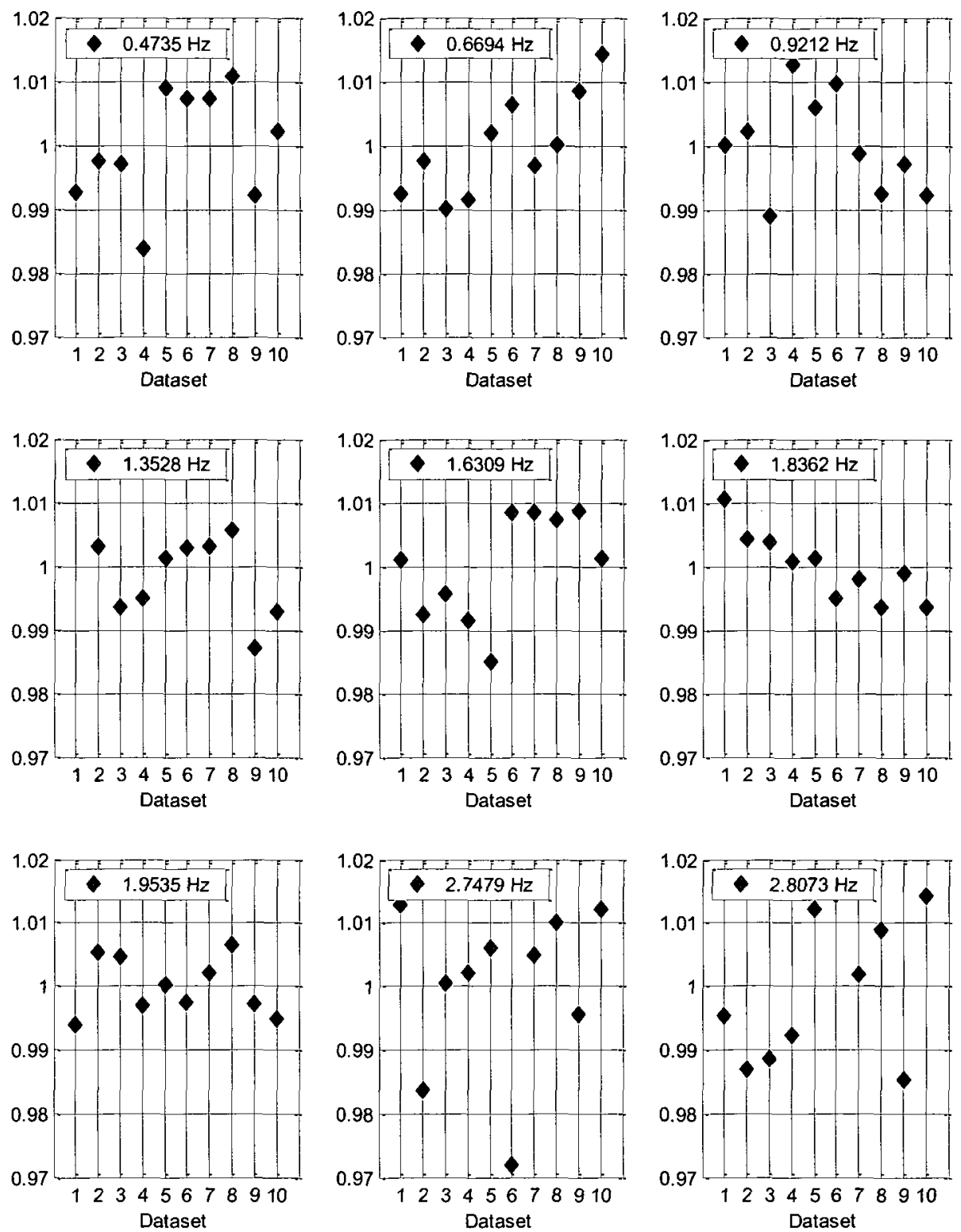

Figure 5-5 : Normalized modal frequencies of baseline variability datasets 

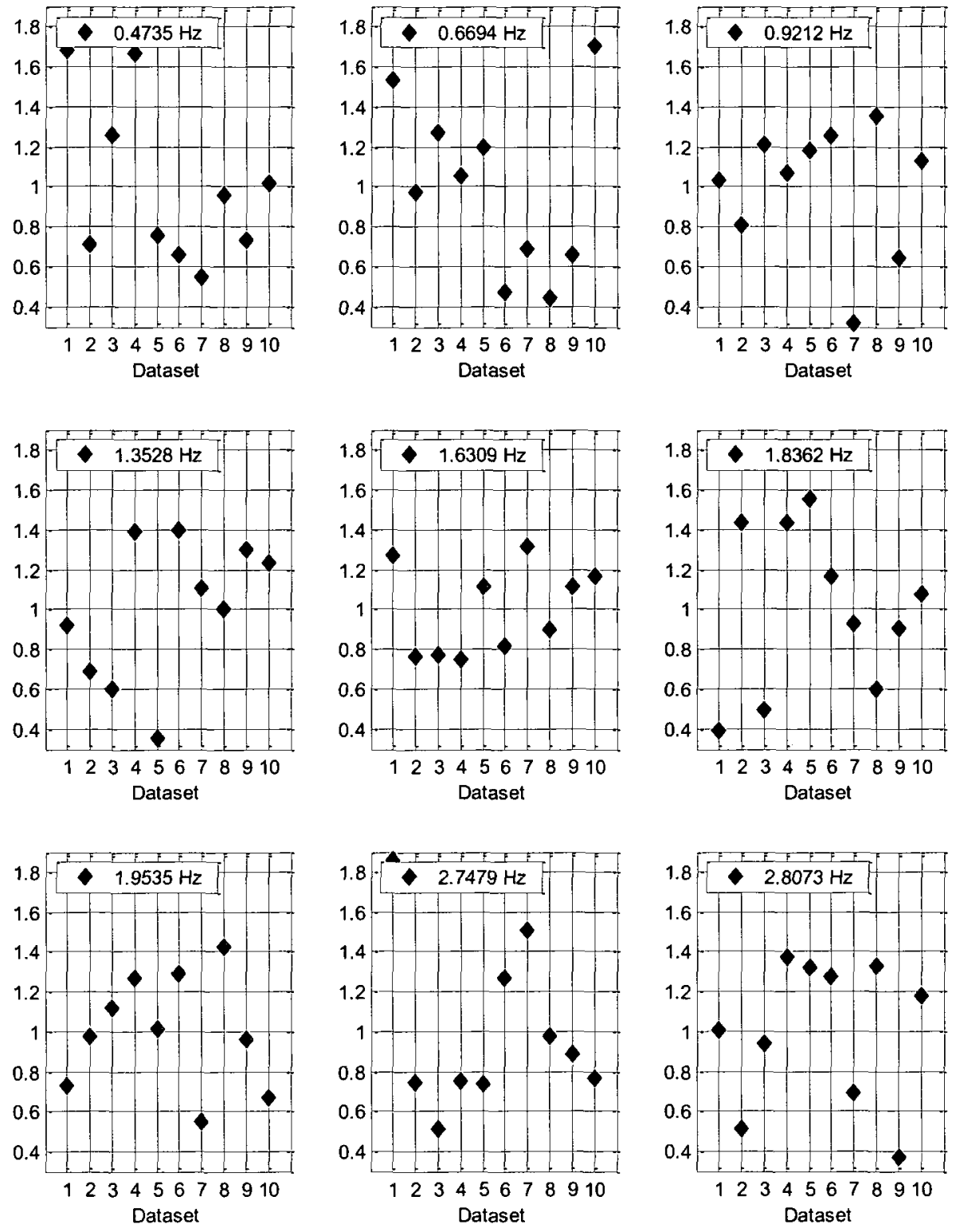

Figure 5-6 : Normalized damping ratios of baseline variability datasets 

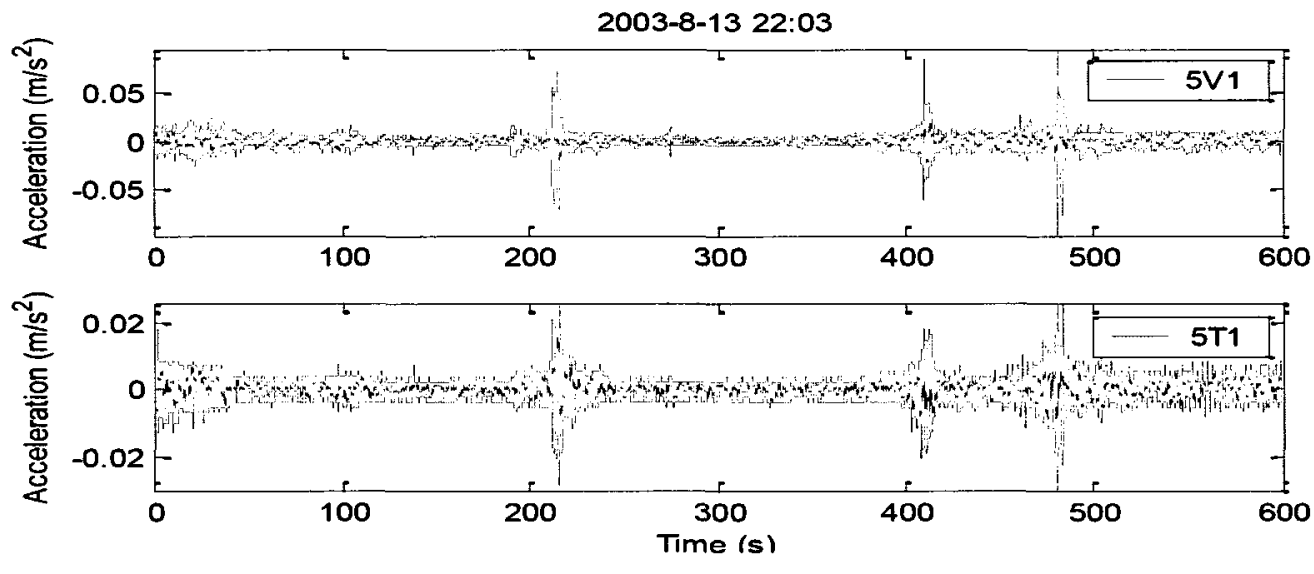

2003-8-25 09:54
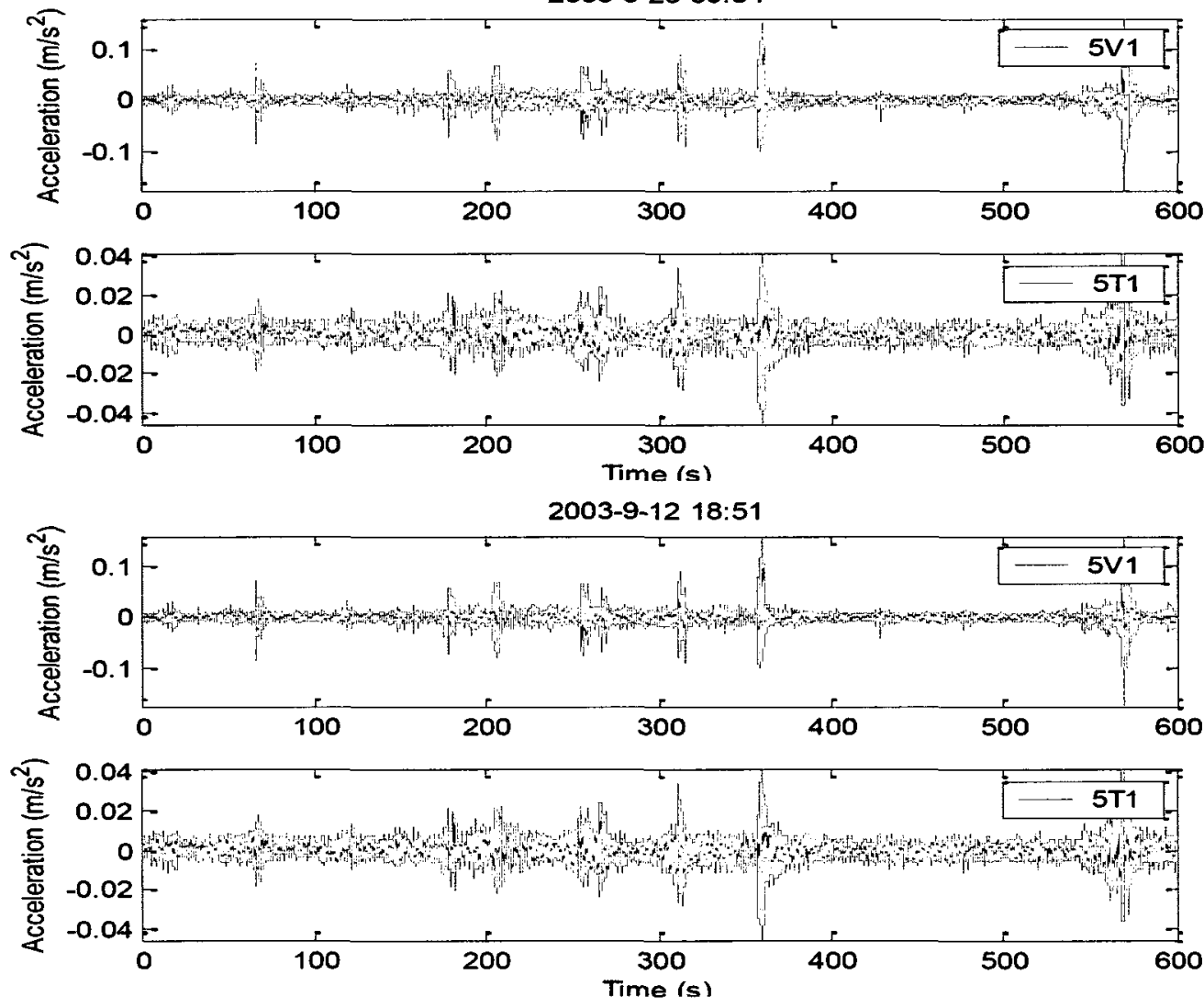

Figure 5-7(A) : Time histories of environmental and loading scenario variability datasets 

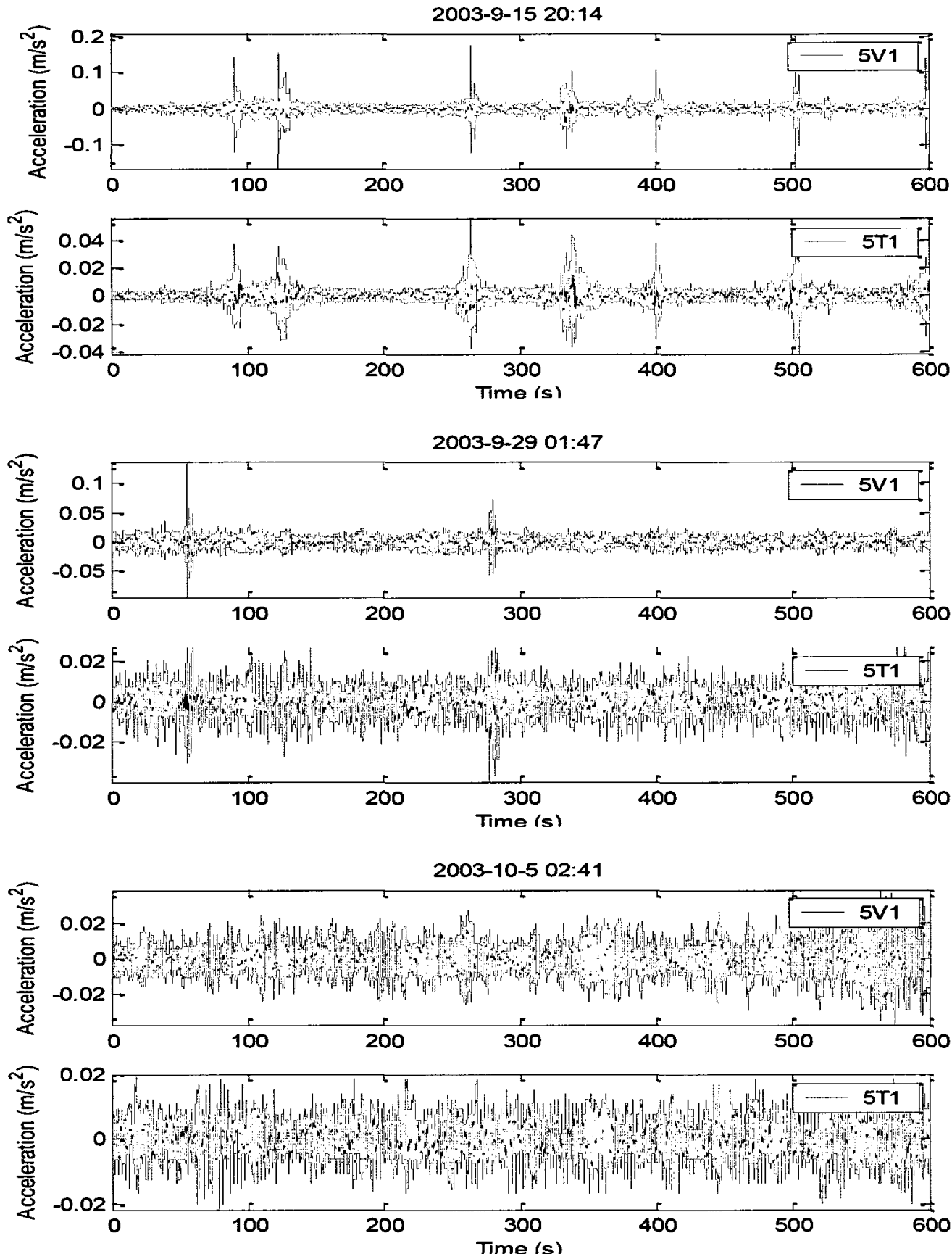

Figure 5-7 (B) : Time histories of environmental and loading scenario variability datasets 
Chapter 5 - Variability of Monitoring Data
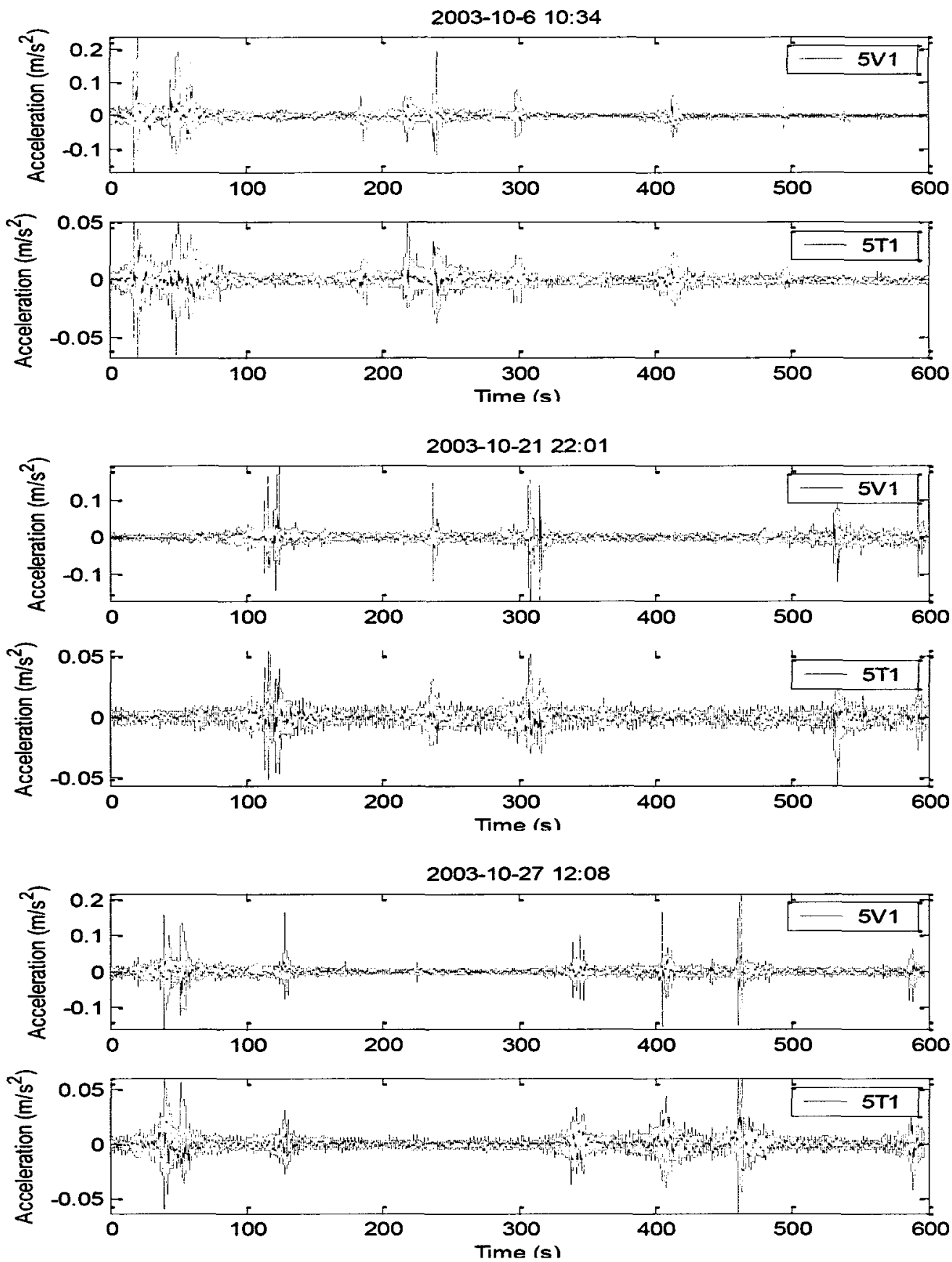

Figure 5-7 (C) : Time histories of environmental and loading scenario variability datasets 
Chapter 5 - Variability of Monitoring Data
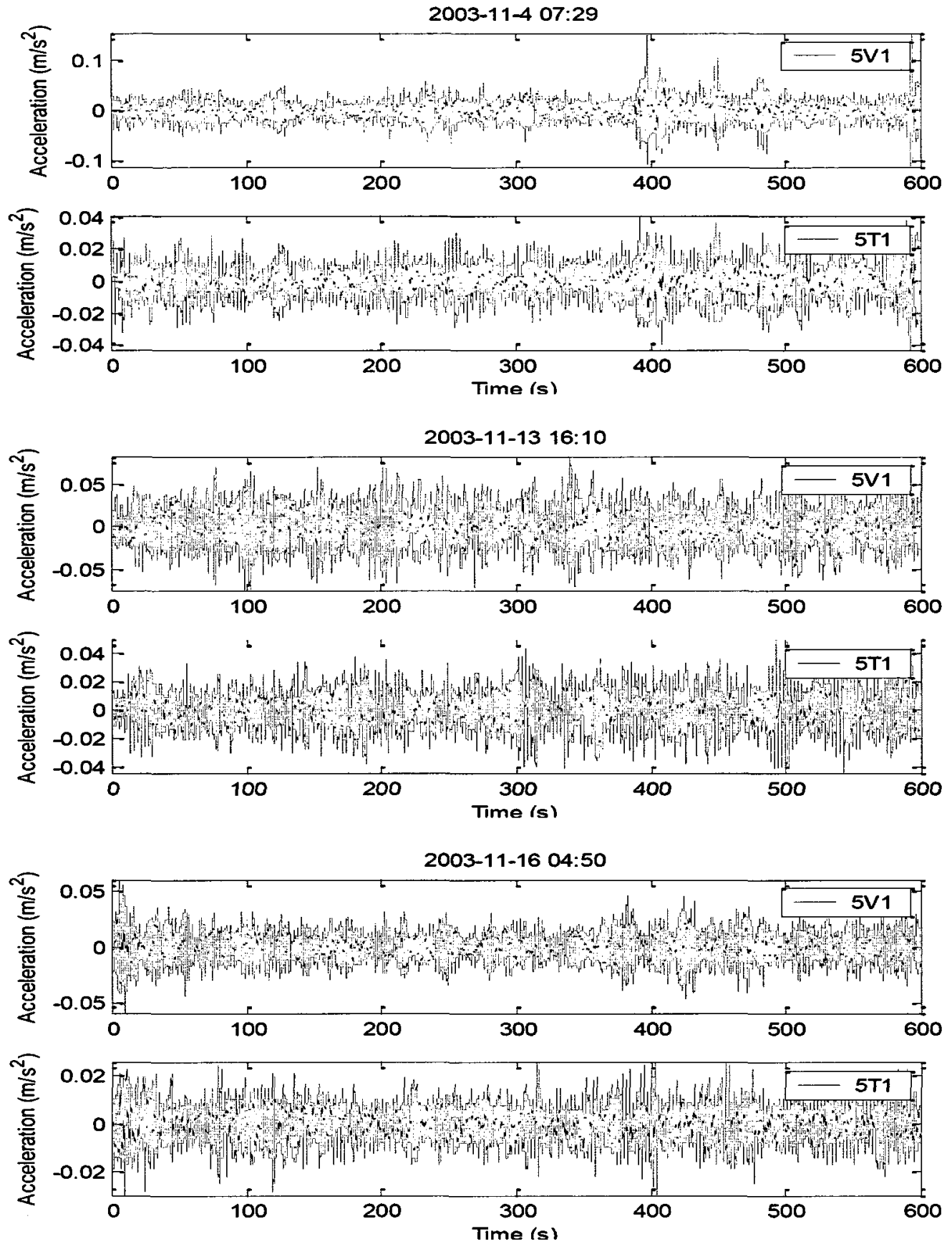

Figure 5-7 (D) : Time histories of environmental and loading scenario variability datasets 

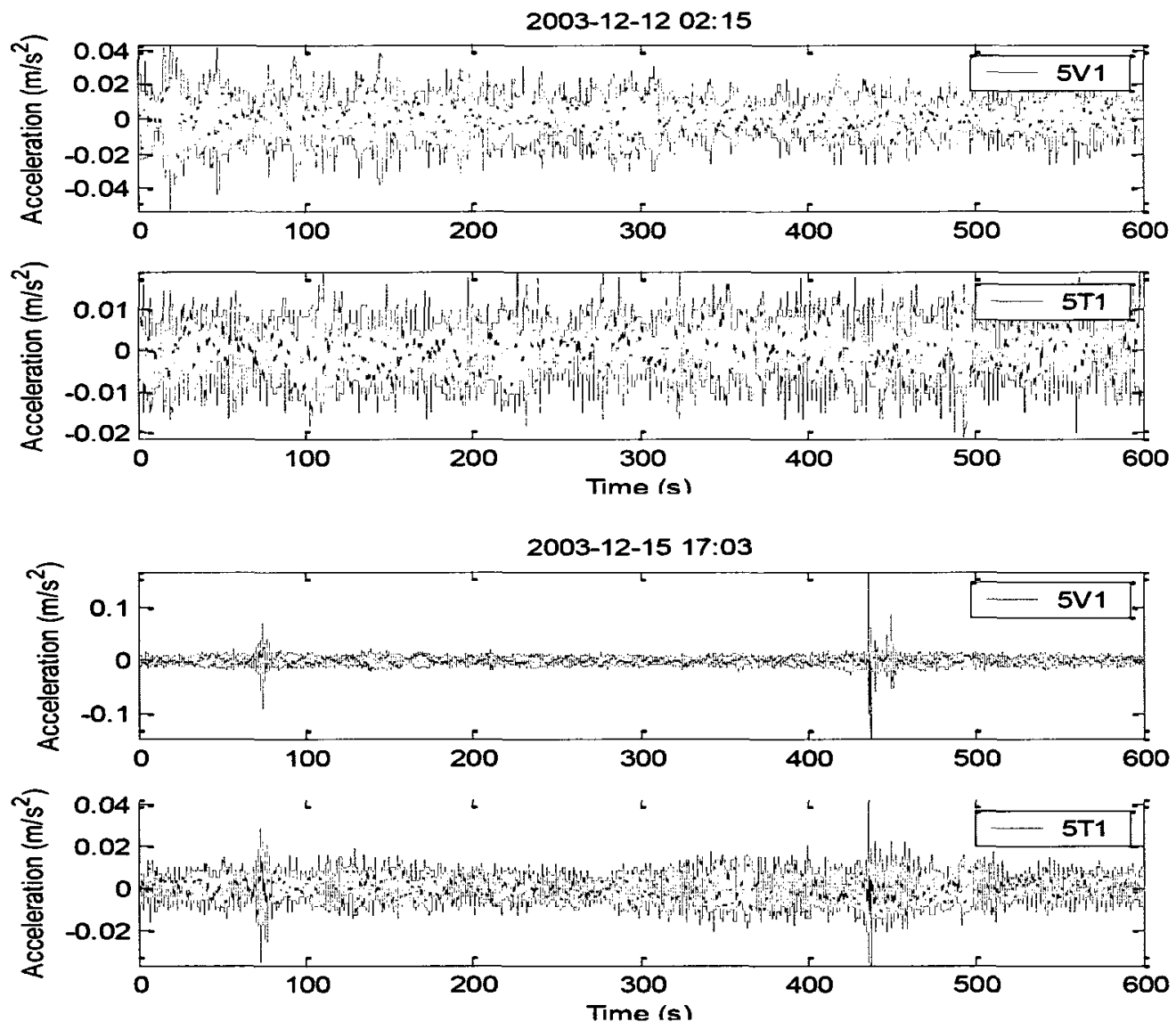

2003-12-16 08:54
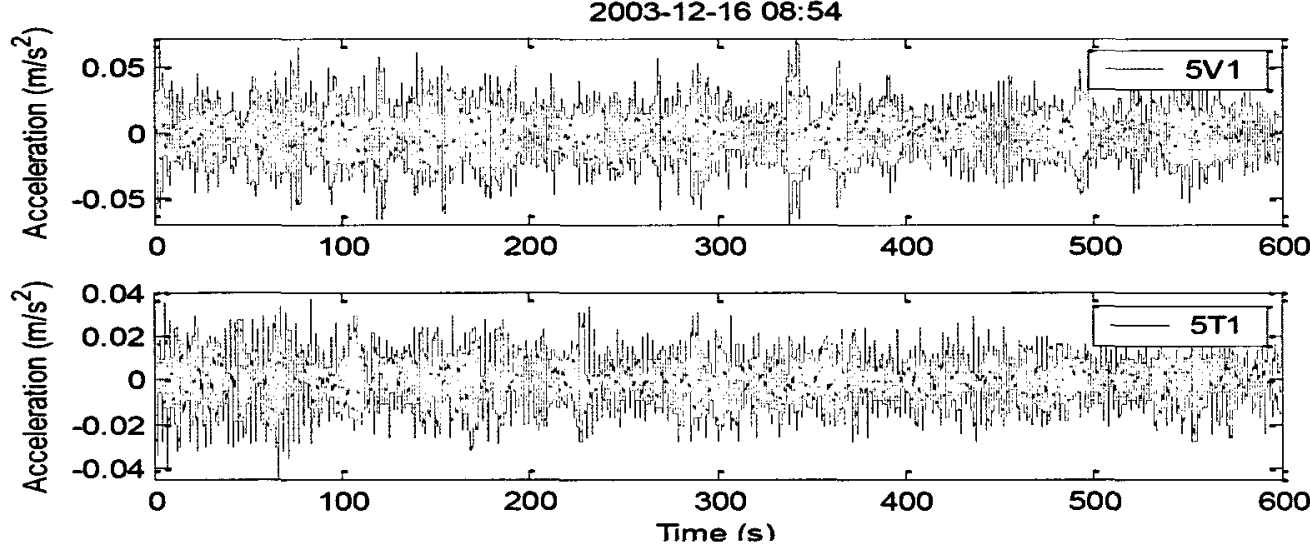

Figure 5-7 (E) : Time histories of environmental and loading scenario variability datasets 

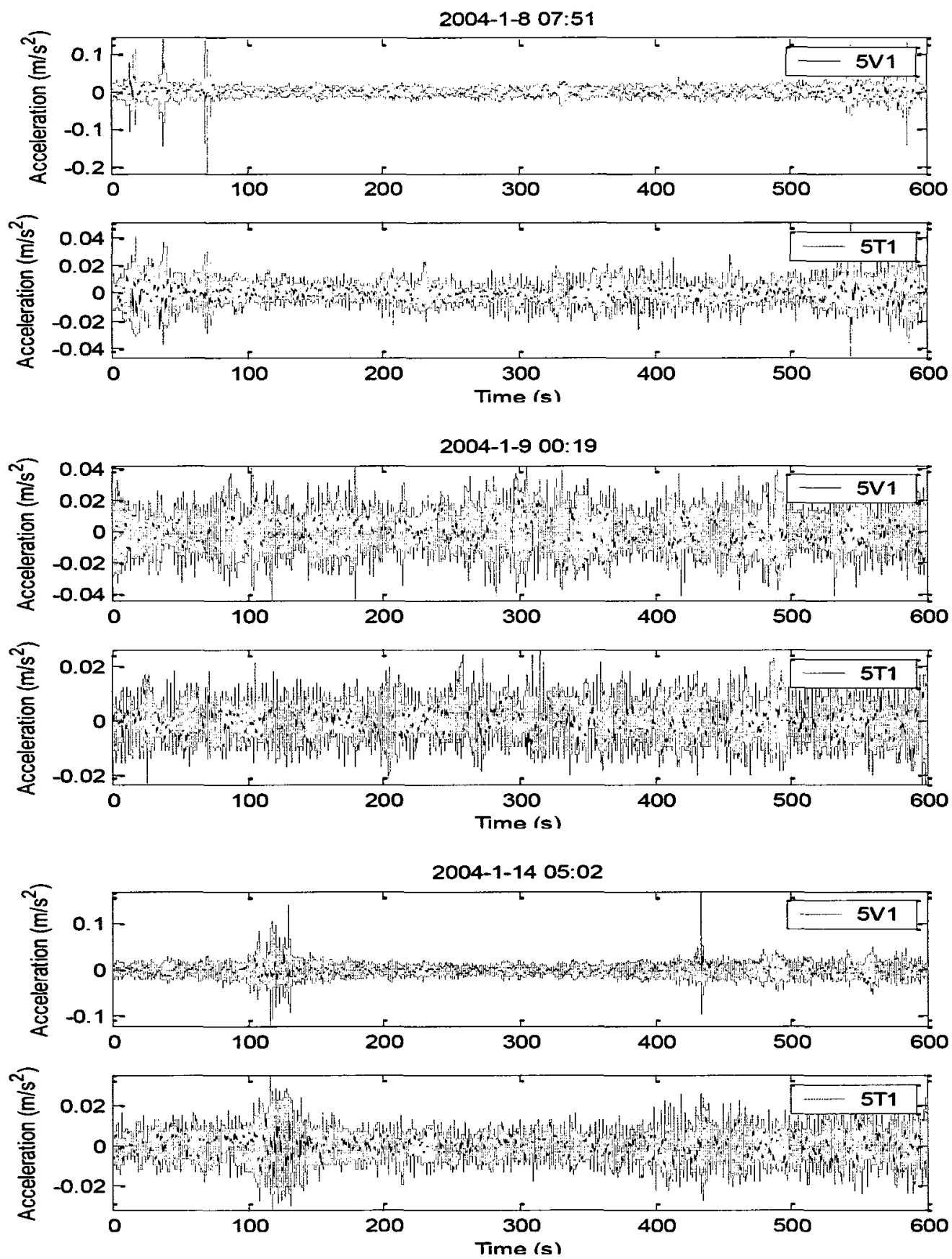

Figure 5-7 (F) : Time histories of environmental and loading scenario variability datasets 

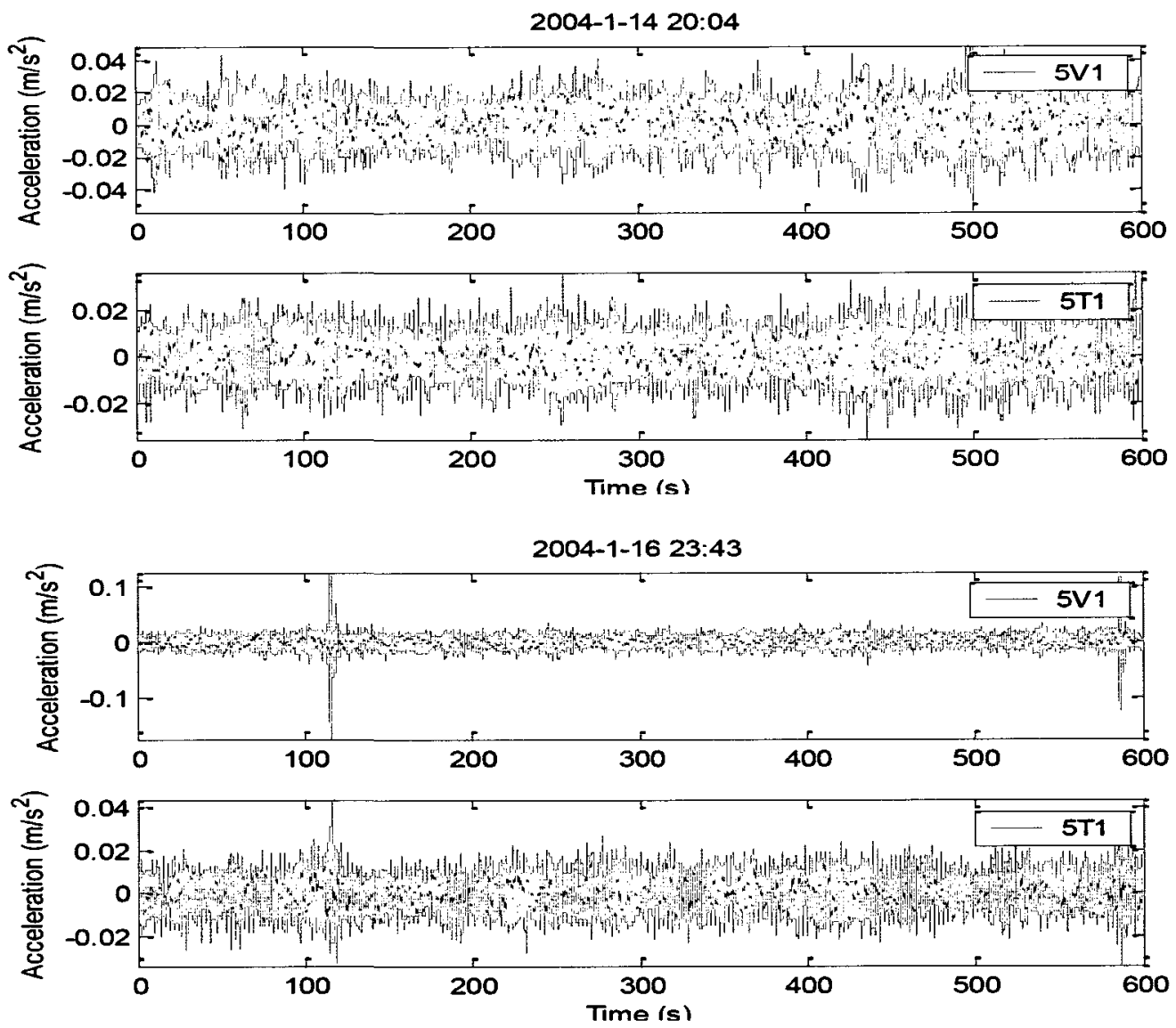

2004-1-17 20:15
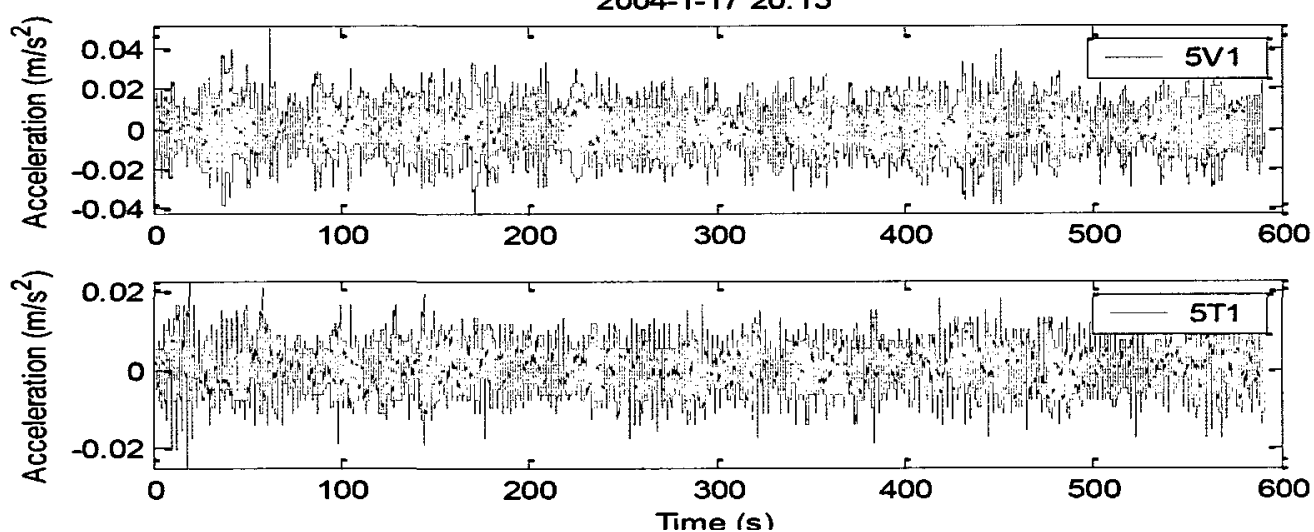

Figure 5-7 (G) : Time histories of environmental and loading scenario variability datasets 

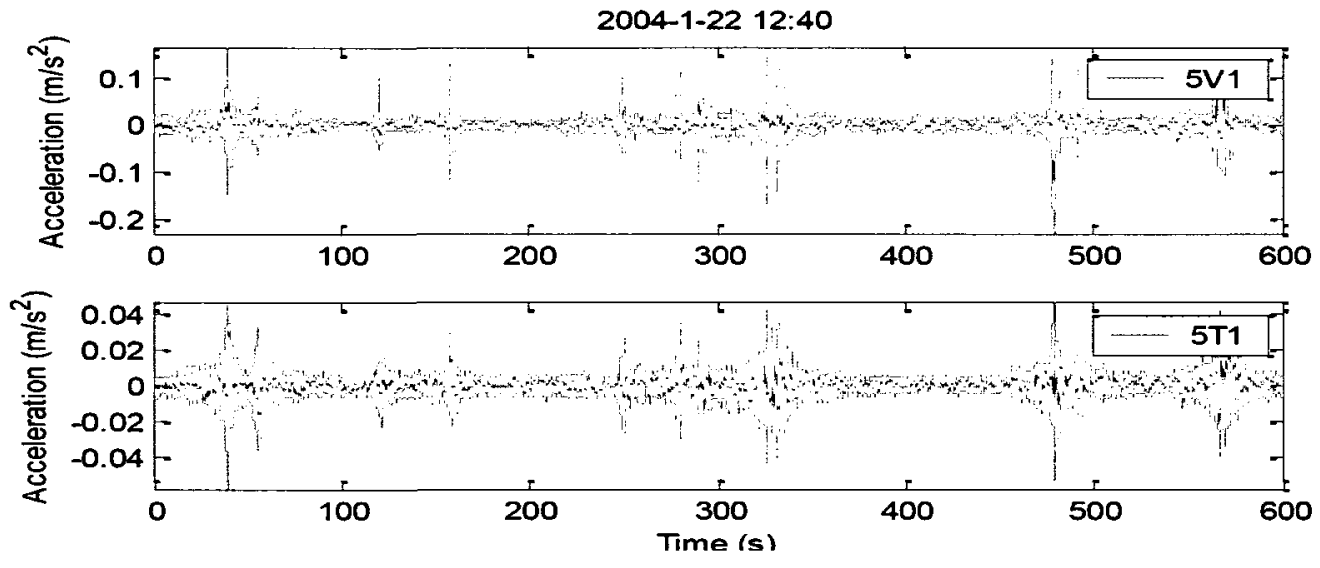

Figure 5-7 (H) : Time histories of environmental and loading scenario variability datasets 


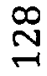
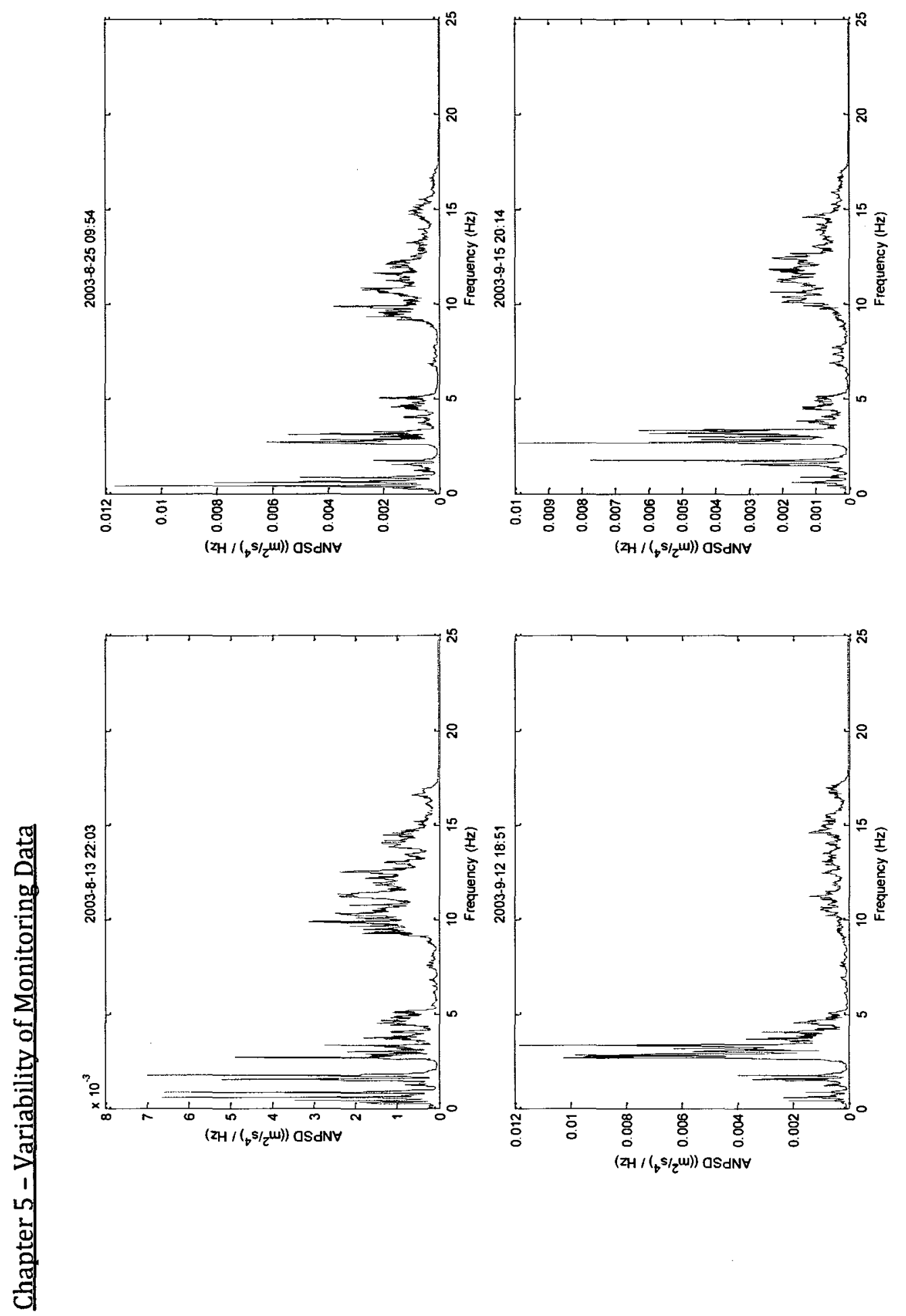

告 
ิำ
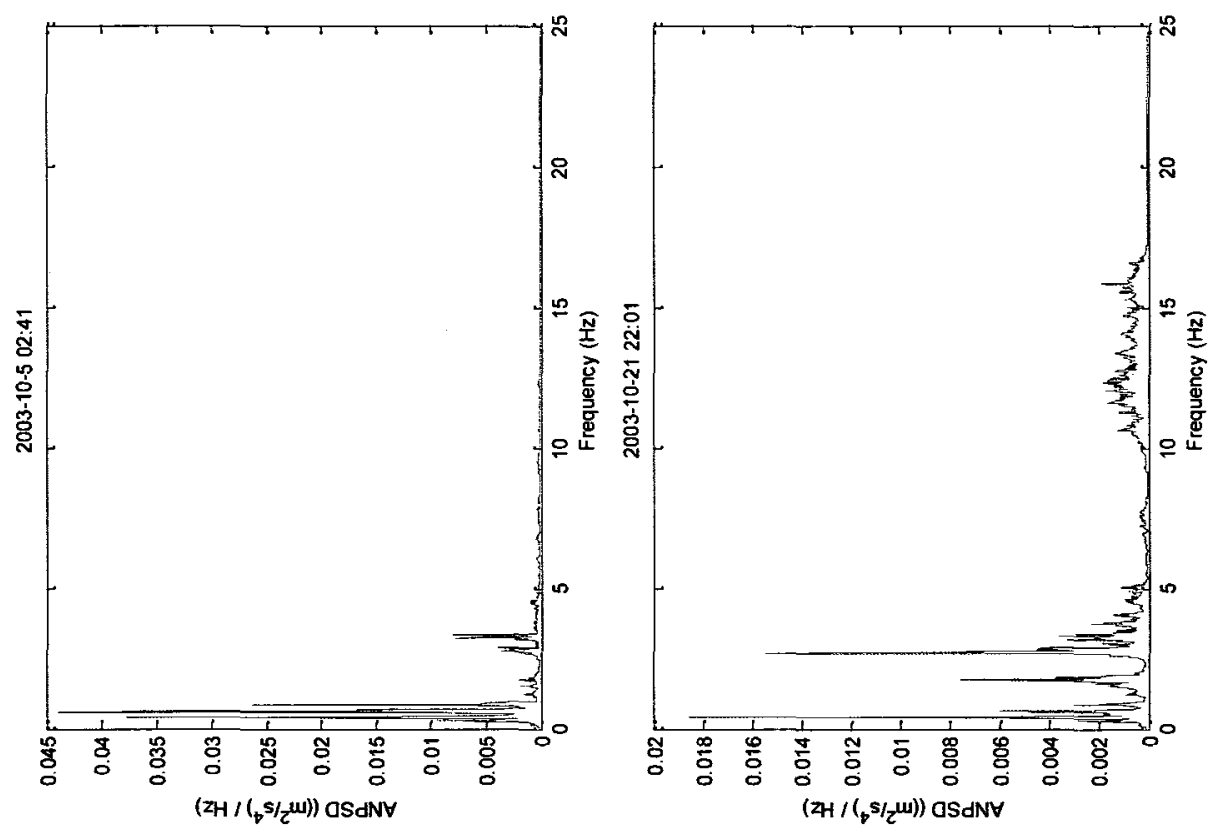

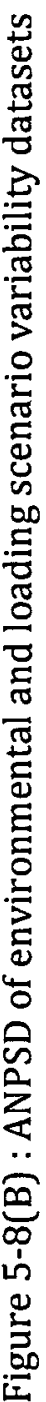

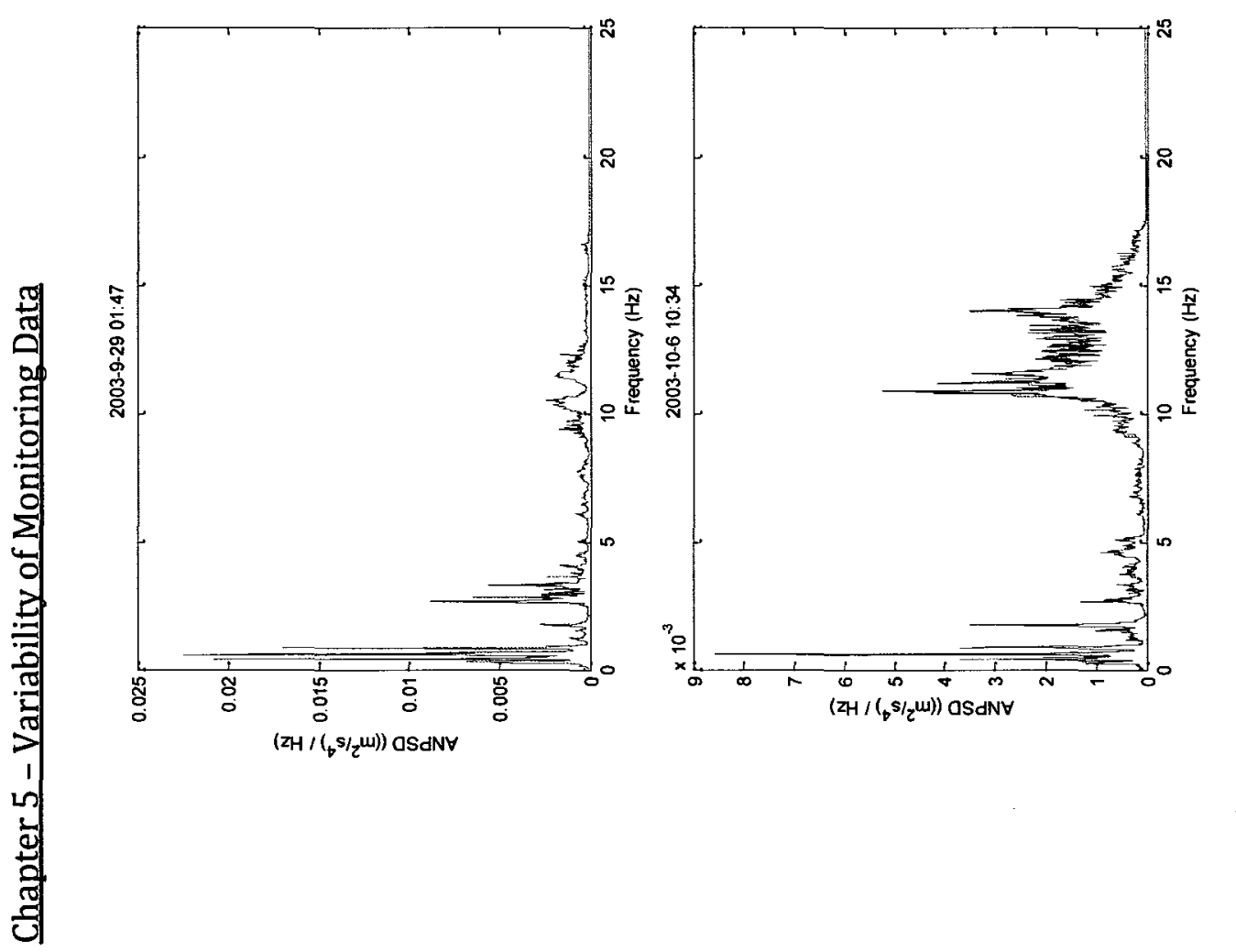


m

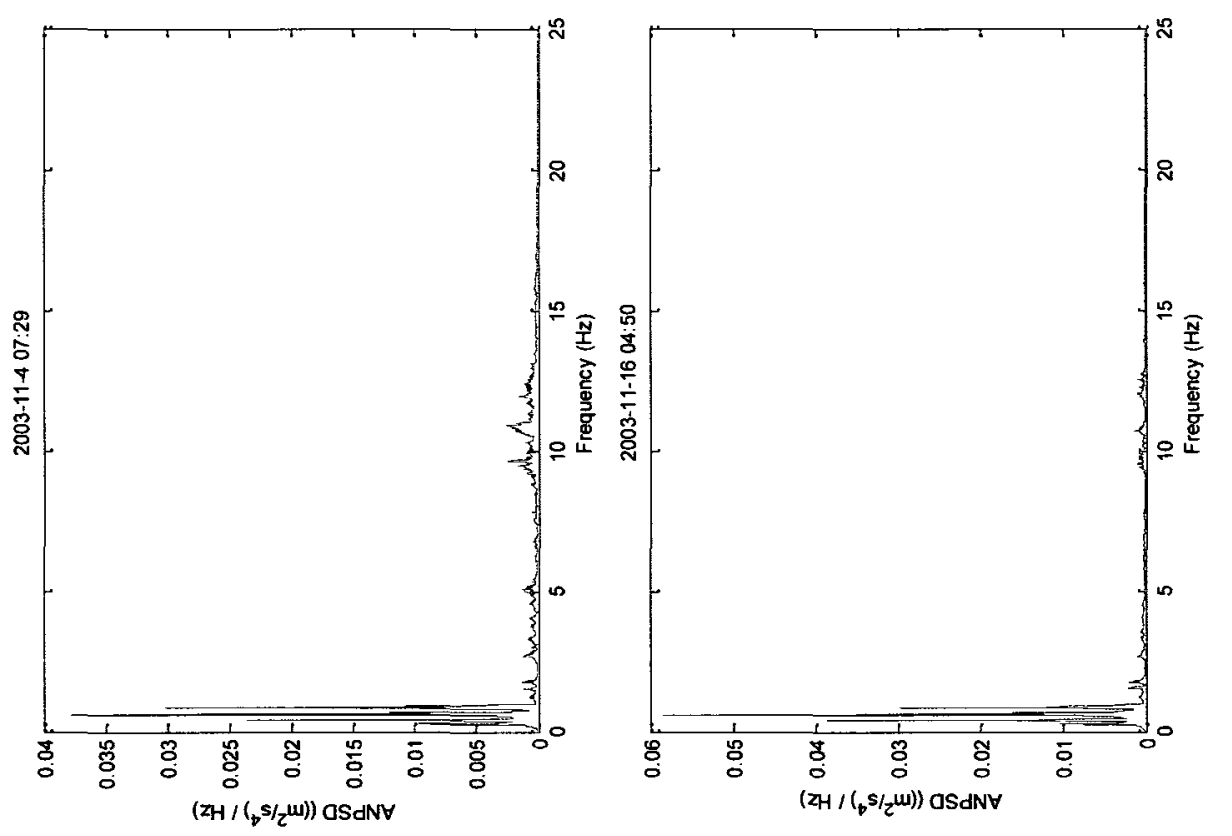

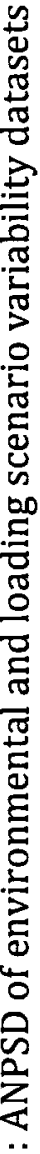

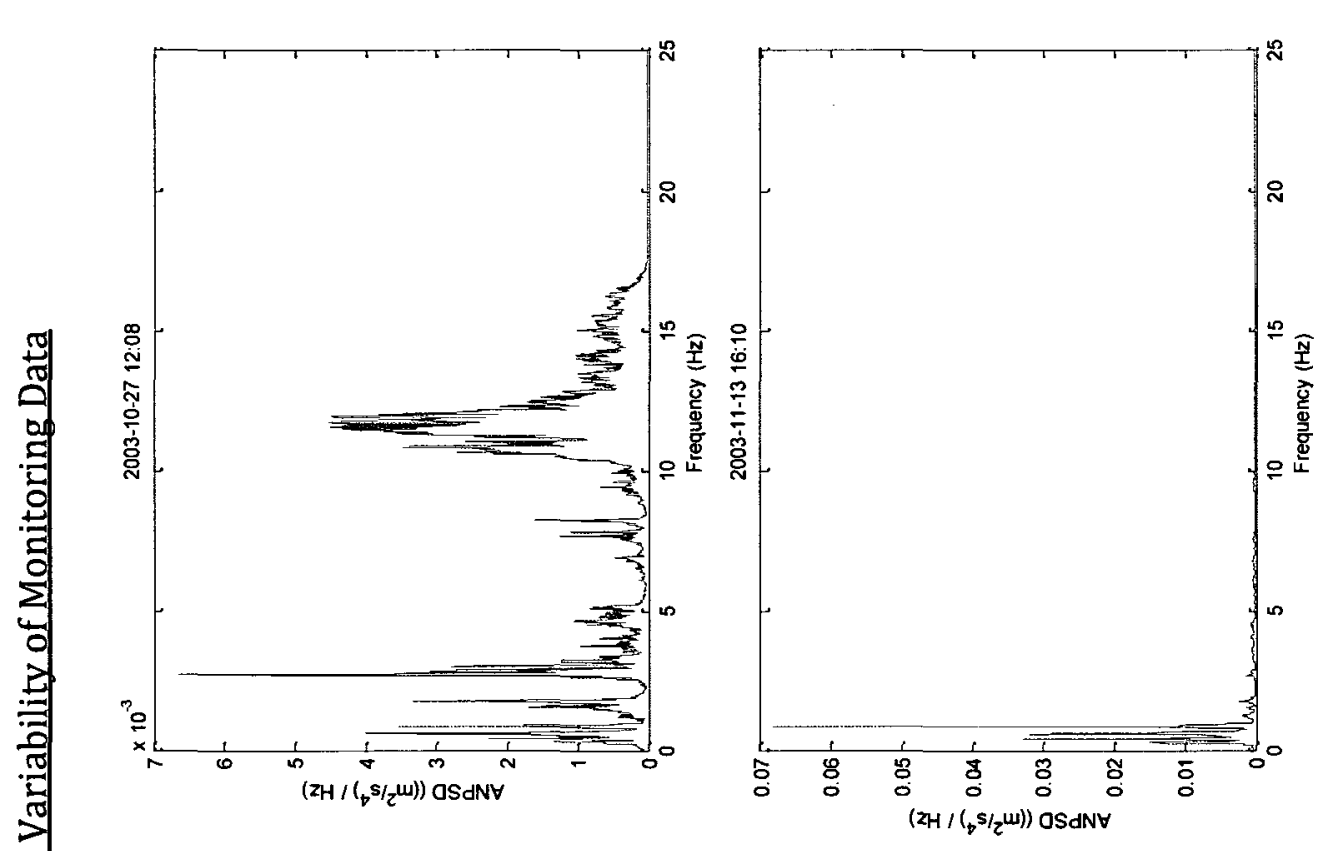

ఠิ

峁 
$\ddot{n}$
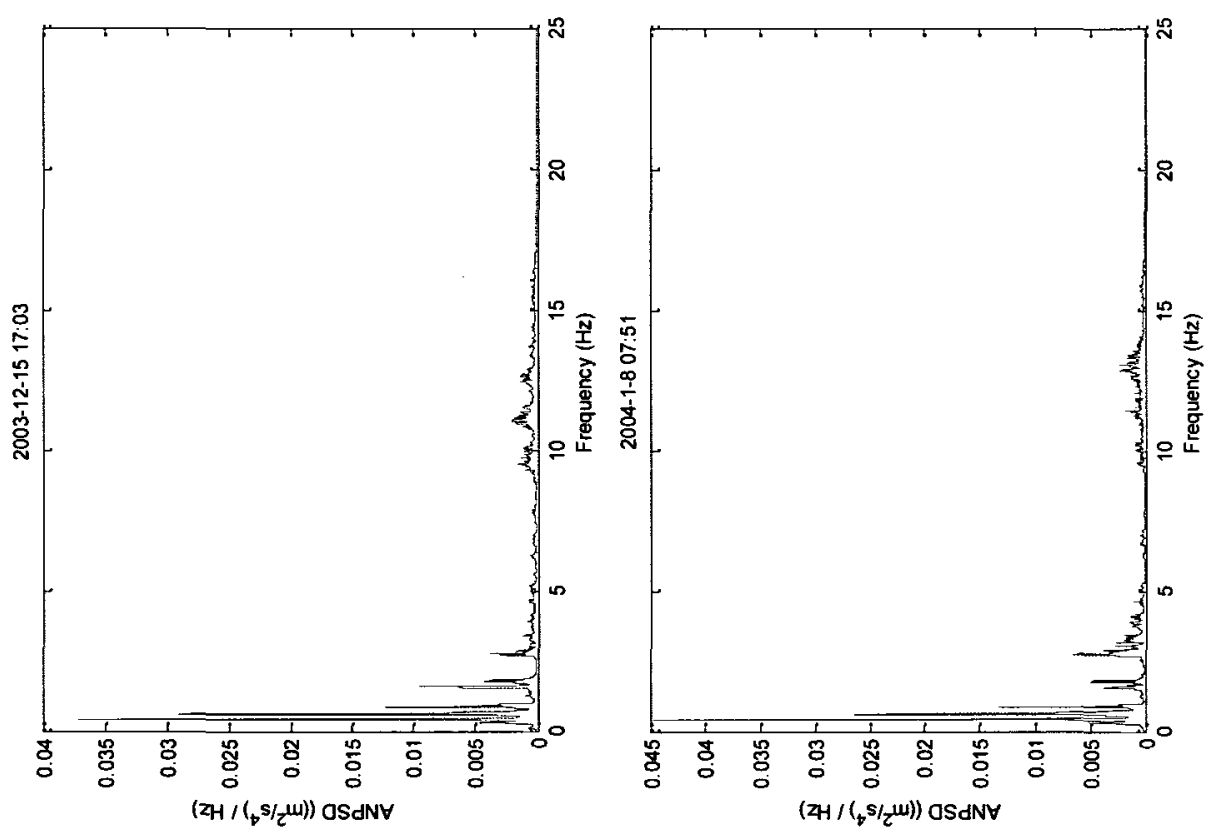

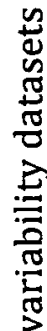

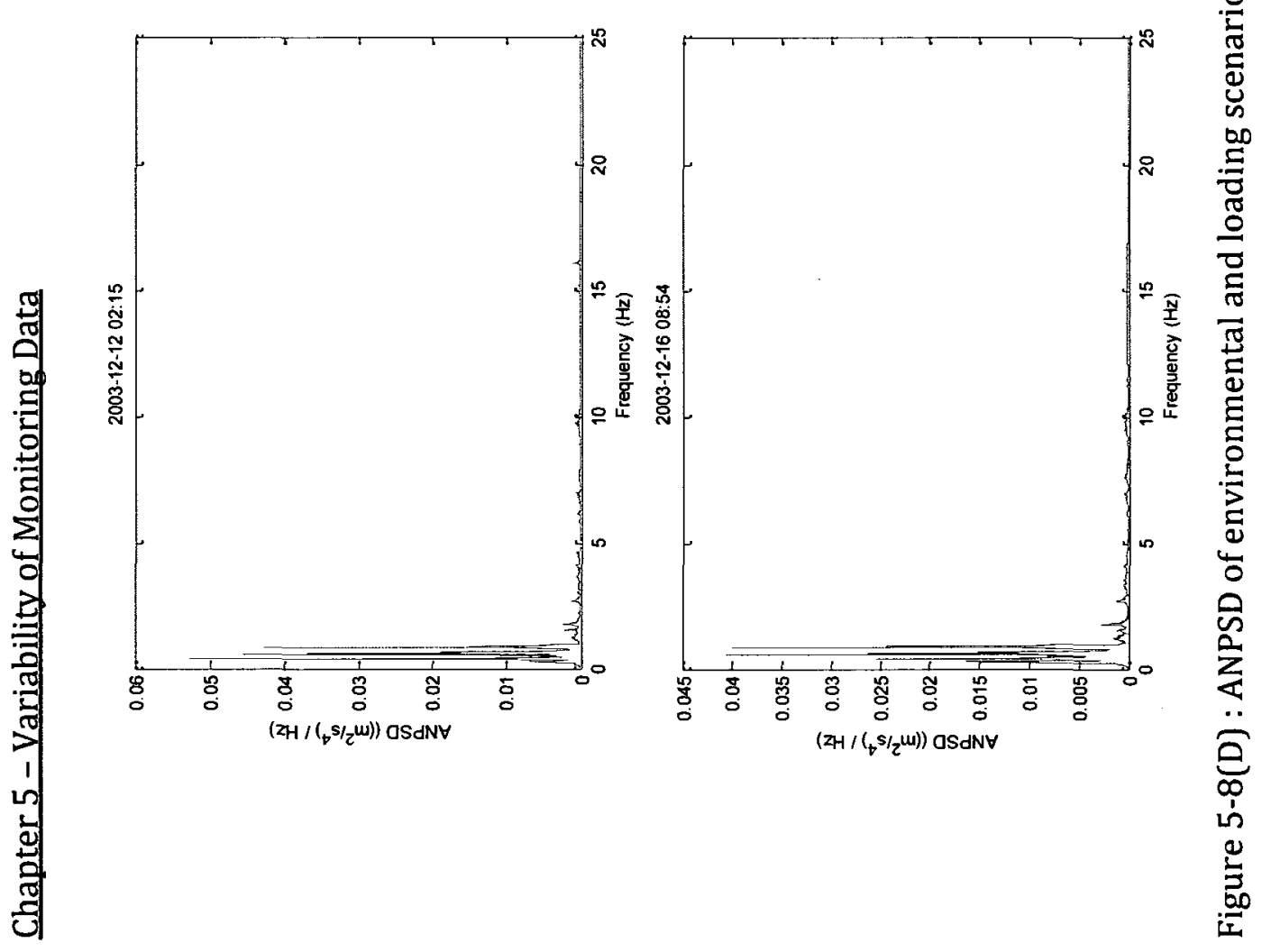


్ㅗ

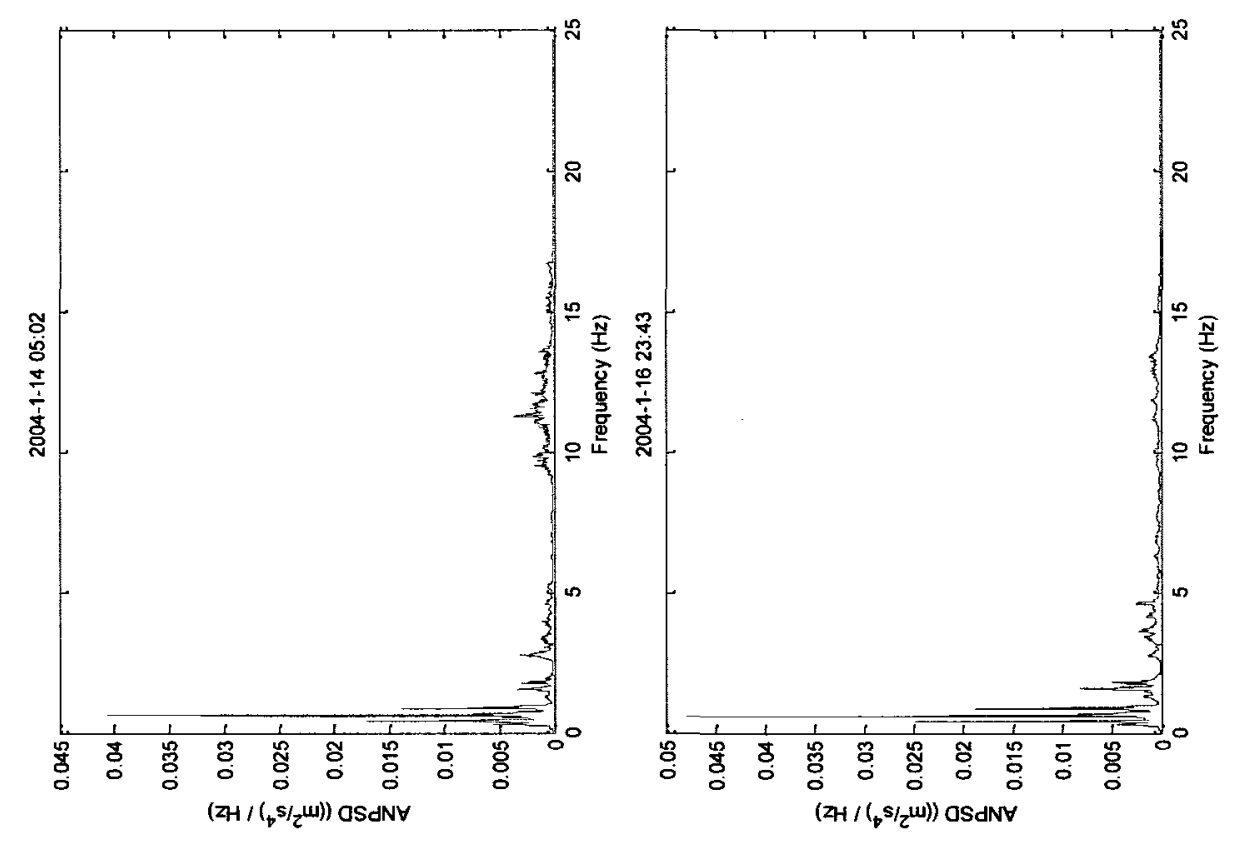



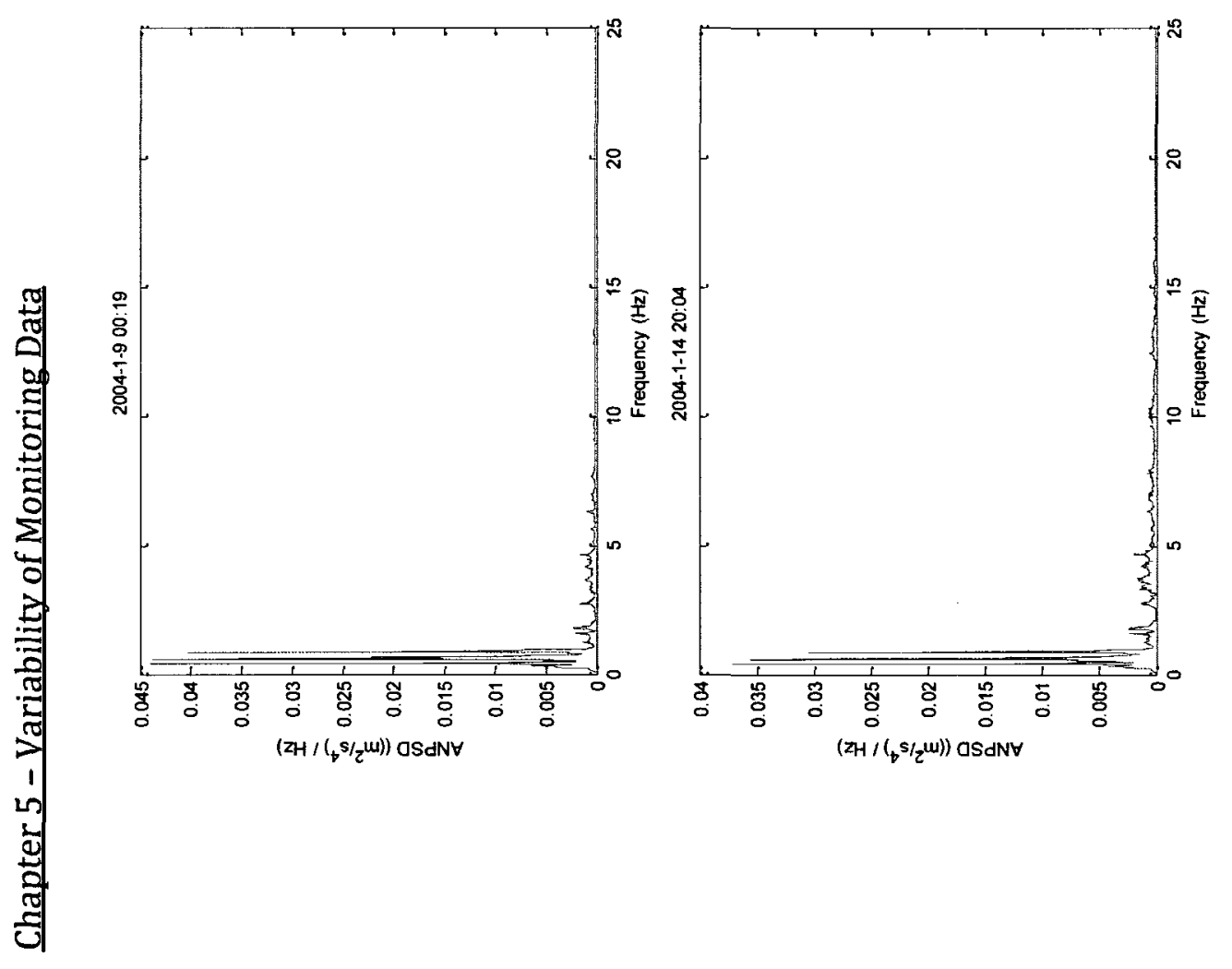

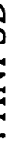


$m$

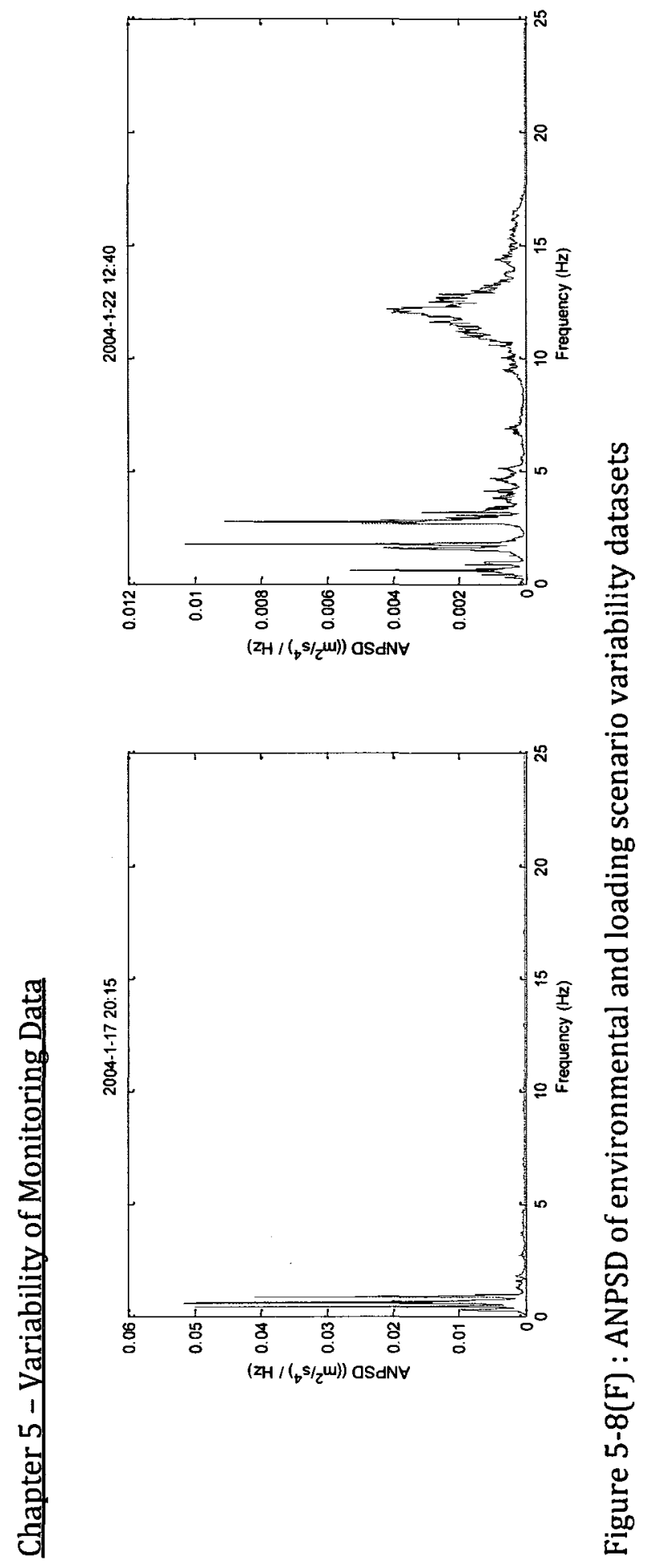


ले
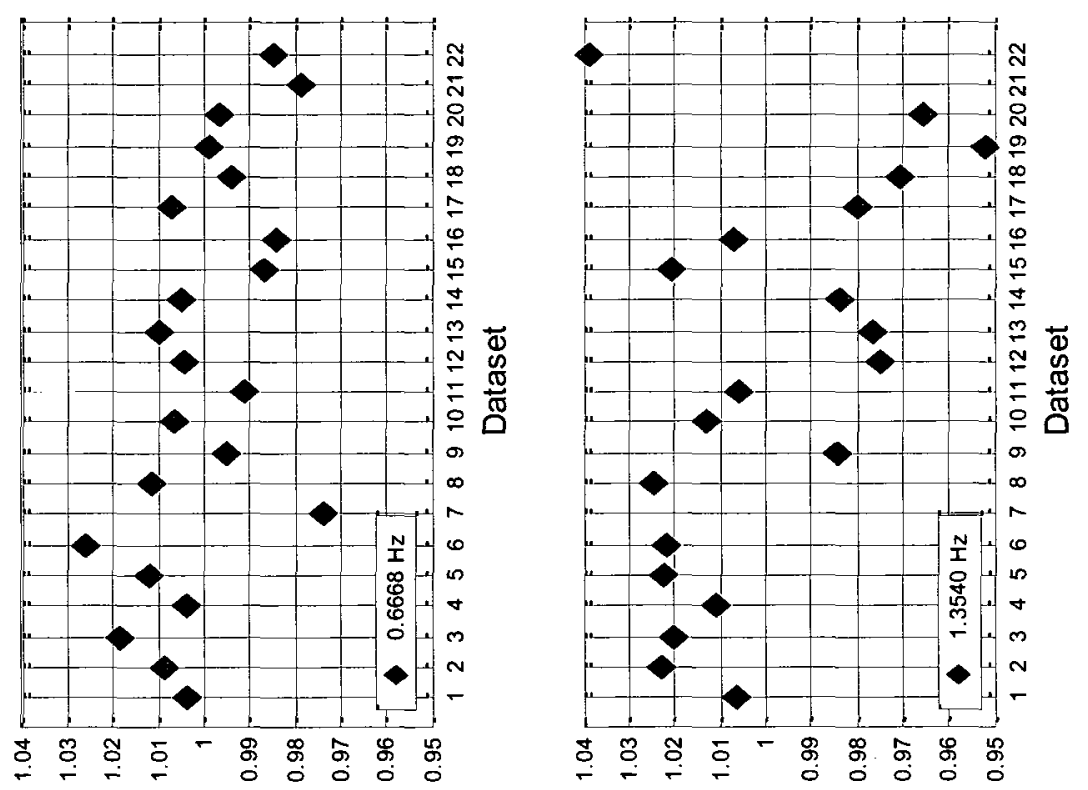

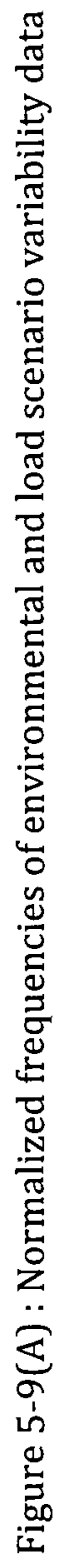


m

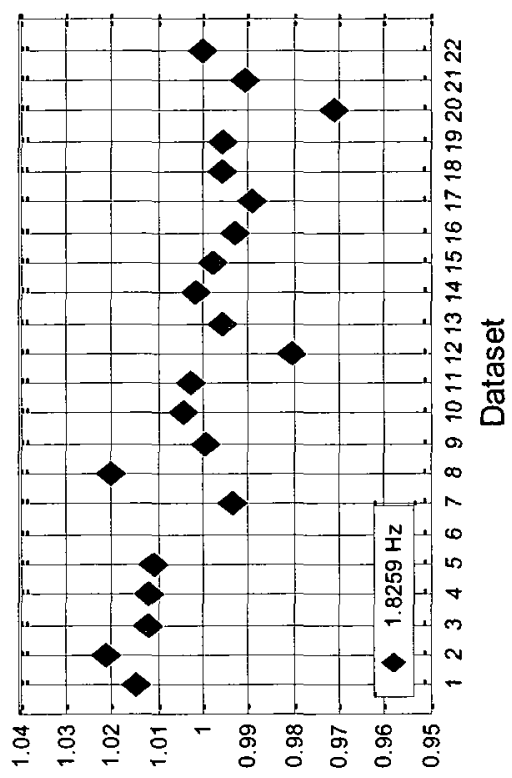

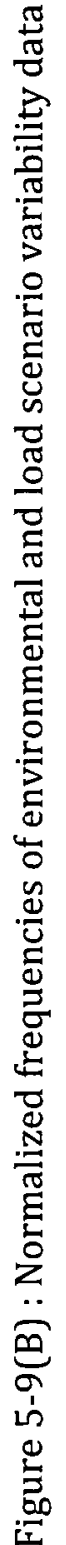




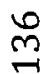
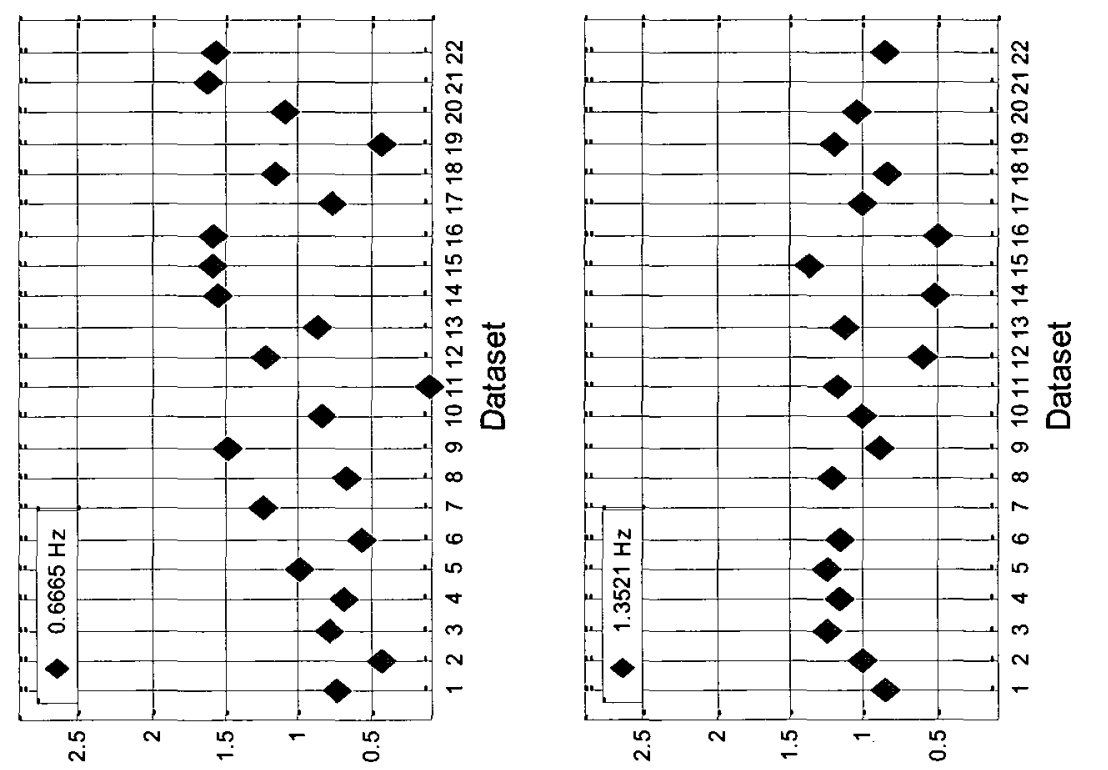

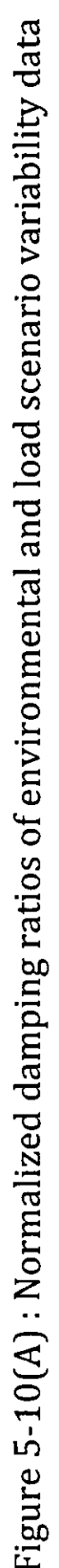
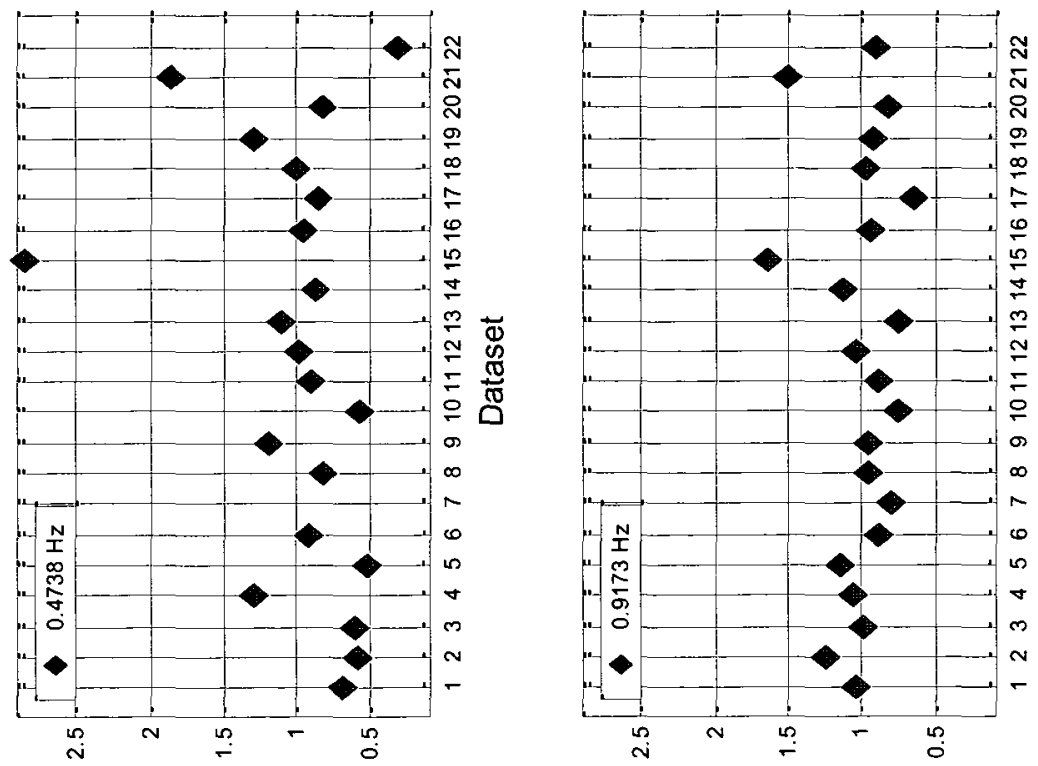
$n$

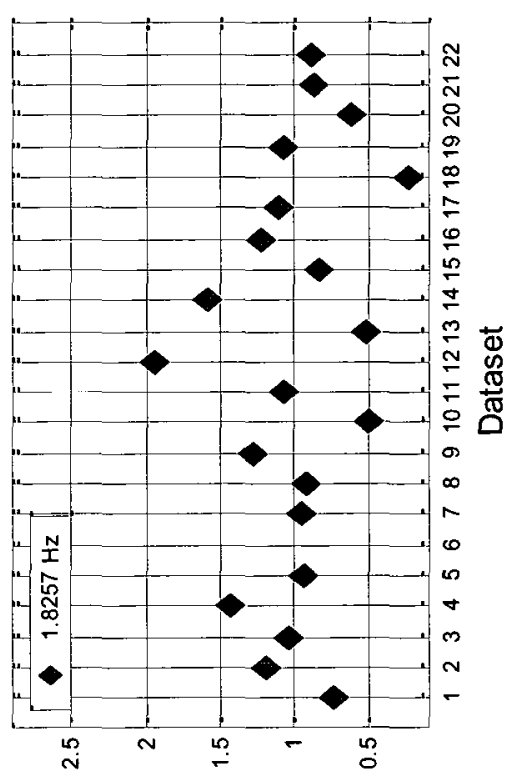

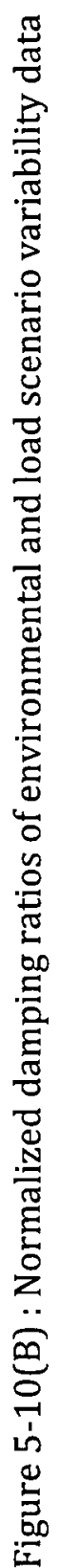




\section{Chapter 6 - Conclusions and}

\section{Recommendations for Future}

\section{Work}

\subsection{Conclusion}

The need for more objective, reliable techniques for structural condition assessment is evident. To this end, vibration based structural health monitoring has garnered significant attention. VBSHM requires the proper instrumentation of structures to capture the necessary response data for condition assessment. This 
instrumentation of a structure generates a large volume of data, especially when one considers the desire to continuously monitor a structure, therefore a software platform, Signal Processing Platform for Analysis of Structural Health, was previously developed. A new, more efficient, system identification module suitable for automatic data analysis of measured vibration response data has been developed. This module incorporates the use of the newly developed polyreference least squares complex frequency estimator for determining the vibration properties of a structure. This new output-only system identification technique has been shown to provide consistent results based on a limited number of reference sensors, allowing for efficient use of computational resources. The clean, well-defined stabilization diagrams provided by the p-LSCF estimator allows for the development of an automated pole selection procedure. This new robust procedure continues to reduce the subjectivity of structural monitoring by eliminating the need of an experienced analyst to determine physical poles of a system from a stabilization diagram. By eliminating this need, the system can run continuously and autonomously, which allows for more accurate results to be obtained in terms of the deterioration or damage of a structure. The accurate and up-to-date engineering information which can now be autonomously extracted from measured vibration response data can lead to more effective and efficient allocation of limited resources during their prioritization for the maintenance and rehabilitation of structures. 


\subsection{Future Work}

Based on reviews of existing software platforms available for structural condition assessment, be they generic platforms or project specific, the following topics are of interest to ensure that SPPLASH is a complete tool for vibration based structural health monitoring, suitable for use in the field by practicing engineers.

\subsubsection{Mode Shape Estimation}

The newly developed system identification technique, polyreference least squares frequency, does not provide the user with mode shapes. However, through the selection of physical poles, which have corresponding operational reference factors, the mode shapes may be estimated by the least square frequency domain (LSFD) method. The incorporation of this algorithm into the newly developed automatic system identification module would complete the module by determining all relevant vibration properties from the data. 


\subsubsection{Damage Detection Module}

An accurate and efficient damage detection module is vital for the progression of vibration based structural health monitoring to a mainstay in the area of monitoring by practicing engineers. Following the current study and previous studies on the variability of data used in the determination of vibration properties, an accurate damage detection algorithm should be developed based on the findings.

\subsubsection{Non-linear System Identification}

The current modules of SPPLASH are developed with linear system identification techniques. While linear assumptions are often valid during design of a structure, structural health monitoring is dependent on the determination of slight variations in the vibration properties of a structure. Therefore the use of a nonlinear system identification technique may be more appropriate so that the general variability of the system is reduced. An extensive database, such as that found compiled for the Confederation Bridge Monitoring Project will facilitate the formulation of such a technique. 


\subsubsection{Engineering Report Module}

To ensure the use of a software platform by all relevant parties, such as bridge owners, operators and or engineers, a module must be developed which provides accurate and relevant engineering information on a consistent basis. This module should provide the user(s) with information of varying scope in the form of weekly, monthly, and annual reports. The automation of the extraction of physical poles from the measured response data should facilitate the development of this module. 


\section{References}

Chan, T., Ko, J., \& Li, Z. (2001). Fatigue evaluation for Tsing Ma bridge using structural health monitoring data. Proceedings of SPIE , 4337, 335-346.

Cheung, M., Tadros, G., Brown, T., Dilger, W., Ghali, A., \& Lau, D. (1997). Field monitoring and research on performance of the Confederation Bridge. Canadian Journal of Civil Engineering , 24 (6), 951 - 962.

Desjardins, S. (2004). Real-time computer platform for vibration-based structural health monitoring of the Confederation Bridge. PhD thesis, Department of Civil \& Environmental Engineering. Carleton University, Ottawa, Canada. DEWESoft. (2007). DEWESoft. Retrieved May 5, 2009, from DEWESoft website: www.dewesoft.org 
Farrar, C. R., Doebling, S. W., Cornwell, P., \& Strase, E. G. (1997). Variability of modal parameters measured on the Alamosa Canyon Bridge. Proceedings of the 15th International Modal Analysis Conference , 257-263.

Federal Highway Administration. (2004). National bridge inspection standards. Federal Regulations.

Felber, A. (1993). Development of a hybrid bridge evaluation system. PhD thesis, Department of Civil Engineering. University of British Columbia, Vancouver, Canada.

Fu, G. (2005). Inspection and monitoring techniques for bridges and civil structures. Cambridge, England: Woodhead Publishing Limited.

Graybeal, B., Rolander, D., Phares, B., Moore, M., \& Washer, G. (2001). Reliability and accuracy of in-depth inspection of highway bridges. Transportation Research Record, 1749, 93-99.

Hawk, H. (1999). BRIDGIT: User-friendly approach to bridge management. International Bridge Management Conference, 1.

Hermans, L., Van der Auweraer, H., \& Guillaume, P. (1998). A frequency-domain maximum likelihood approach for the extraction of modal parameters from output-only data. Proceedings of ISMA23, the International Conference on Noise and Vibration Engineering, (pp. 367-376).

Heylen, W., Lammens, S., \& Sas, P. (1997). Modal analysis theory and testing. KU. Leuven, Belgium. 
Kriviak, G. (1999). Bridge management system development for municipal-sized inventories in Western Canada. 8th International Bridge Management Conference, 1 .

Lanslots, J., Rodiers, B., \& Peeters, B. (2004). Automated pole-selection: proof-ofconcept \& validation. Proceedings of ISMA , 1005-1018.

Li, H., Ou, J., Zhao, X., Zhou, W., Li, H., \& Zhou, Z. (2006). Structural health monitoring system for the Shandong Binzhou Yellow River highway bridge. ComputerAided Civil and Infrastructure Engineering , 21, 306-317.

Li, N., Zhang, X., Zhou, X., Leng, J., Liang, Z., \& Zheng, C. (2008). Introduction of structural health and safety monitoring warning systems for Shenzhen Hongkong western corridor Shenzhen bay bridge. Proceedings of SPIE, 6935.

Londono, N. (2006). Use of vibration data for structural health monitoring of bridges. PhD thesis, Department of Civil \& Environmental Engineering. Carleton University, Ottawa, Canada.

MacGregor, J., Kennedy, D., Bartlett, F., Chernenko, D., Maes, M., \& Dunaszegi, L. (1997). Design criteria and load and resistance factors for the Confederation Bridge. Canadian Journal of Civil Engineering , 24 (6), 882-897.

Marple, S. (1987). Digital Spectral Analysis with Applications. Englewood Cliffs: Prentice-Hall Inc.

Ndambi, J. M., Peeters, B., Maeck, J., Visscher, J. D., Wahab, M. A., Vantomme, J., et al. (2000). Comparison of techniques for modal analysis of concrete structures. Engineering Structures , 22 (9), 1159-1166. 
Parloo, E. (2003). Application of frequency-domain system identification techniques in the field of operational modal analysis. PhD Thesis, Department of Mechanical Engineering. V.U. Brussel, Belgium.

Peeters, B. (2000). System identification and damage detection in civil engineering. PhD thesis, Department of Civil Engineering. KU, Leuven, Belgium.

Peeters, B., \& Ventura, C. (2003). Comparative study of modal analysis techniques for bridge dynamic characteristics. Mechanical Systems and Signal Processing $, 15(5), 965-988$.

Peeters, B., Couvreur, G., Razinkov, O., Kundig, C., Van der Auweraer, H., \& De Roeck, G. (2003). Continuous monitoring of the Oresund Bridge: system and data analysis. Proceedings of IMAC XXI, A conference and exposition on structural dynamics .

Peeters, B., Maeck, J., \& De Roeck, G. (2001). Vibration-based damage detection in civil engineering: excitation sources and temperature effects. Smart Materials and Structures, $10,518-527$.

Peeters, B., Van der Auweraer, H., Guillaume, P., \& Leuridan, J. (2004a). The PolyMAX frequency-domain method: a new standard for modal paramter estimation? Shock and Vibration, 11, 395-409.

Peeters, B., Van der Auweraer, H., Vanhollebeke, F., \& Guillaume, P. (2007). Operational modal analysis for estimating the dynamic properties of a stadium structure during a football game. Shock and Vibration , 14, 283-303. 
Peeters, B., Vecchio, A., \& Van der Auweraer, H. (2004b). PolyMAX modal parameter estimation from operational data. Proceedings of the 2004 International Conference on Noise and Vibration Engineering , 1049-1063.

Robert, W., Marshall, A., Shepard, R., \& Aldayuz, J. (2003). Pontis bridge management system : state of the practice in implementation and development. 9th International Bridge Management Conference , 49-60.

Speiran, K., Francis, J., Ellis, R., \& Thompson, P. (2004). Implementation of a bridge management system in the province of Nova Scotia. Annual Conference of the Transportation Association of Canada. Quebec City.

Strainstall. (2005). Bridge Monitoring. Retrieved May 12, 2009, from Strainstall Web site: www.strainstall.com/bridge.html

Thompson, P., Ellis, R., Hong, K., \& Merlo, T. (2003). Implementation of Ontario Bridge Management System. 9th International Bridge Management Conference, $112-127$.

Van der Auweraer, H., \& Peeters, B. (2004). Discriminating physical poles from mathematical poles in high order systems: use and automation of the stabilization diagram. Instrumentation and Measurement Technology Conference, , 2193-2198.

Verboven, P. (2002). Frequency-domain system identification for modal analysis. PhD thesis, Department of Mechanical Engineering. V.U. Brussel, Belgium. Vienna Consulting Engineers. (2008). About Brimos. Retrieved May 5, 2009, from Brimos A Product of VCE Web site: www.brimos.com/Brimos 
Welch, P. (1967). The use of fast fourier transform for the estimation of power spectra: a method based on time averaging over short, modified periodograms. IEE Transactions on Audio and Electroacoustics , 15 (2), 70-73.

Wong, K. (2003). Structural identification of Tsing Ma bridge. Transaction Hong Kong Institution of Engineers , 38-47. 